Historic, Archive Document

Do not assume content reflects current scientific knowledge, policies, or practices. 



\section{SEED CATALOG} For

THE DISCRIMINATING FLORIST

$S E A S O N O F \quad 1927-1928$

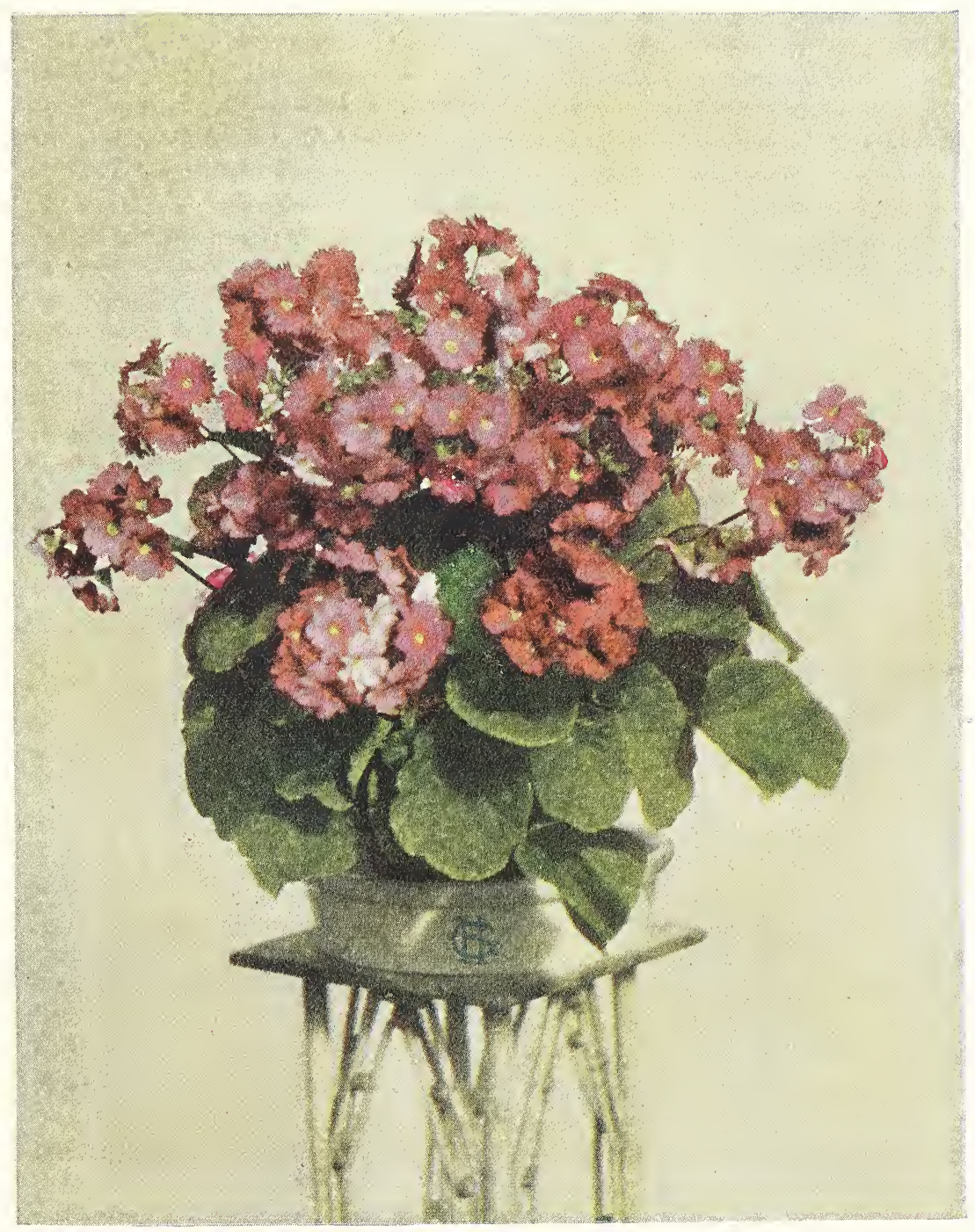

PRIMULA OBCONICA "RERLIN SPECIALTY"

ISSUED BY

\section{HERMANN GLIEME}

1824 FOSTER AVE., CHICAGO, U.S.A. 



\section{FOREWORD}

It is with great pleasure that I am sending to my customers this new price list. Many thanks are tendered old customers who have shown their approval of the quality of my seeds and my methods of serving them by repeated orders.

It shall always be my endeavor to supply you only with the very best seeds from European markets and my aim is to make my prices as low as is possible with high grade quality.

lt is not always possible to deliver seeds at a definite date owing to the fact that the crops depend very much on weather conditions. In such cases a delay of necessity must occur; however, all orders will be filled as quickly as possible. I guarantee my stock to be absolutely fresh and for this reason of superior germinating qualities. 


\section{TERMS OF SALE}

Cash should accompany all orders of $\$ 50.00$ or less, if not, goods will be shipped C. O.D. I will, however, open accounts for orders amounting to more than $\$ 50.00$ if credit is established. In such cases all accounts must be paid for by the first of the month following date of purchase. Any seeds not listed in the catalog will be procured for you direct from European Seed Centers. The time required is usually from eight to ten weeks.

All price quotations in this catalog will be void with the issue of a new catålog.

Yours for better seeds,

HERMANN GLIEME. 


\section{FLOWER - SEEDS}

\section{ABUTILON}

Will blossom at any time of the year under glass and out of doors during summer and autumn.

Hybridum atrosanguineum, dark blood red.

1/128 oz. 1/64 oz. 1/32 oz.

“ maximum, new giant free flowering hybrids........... 75

“ roseum globosum, globular, rose.............. .50

$\$ 1.00$

$\$ 2.00$

“ finest mixed

.50

1.50

3.00

$1.00 \quad 2.00$

$1.00 \quad 2.00$

\section{ACACIA}

Arabica .

Armata, yellow......................... . 30

$\$ 0.50$

Catechu.

Cavenia

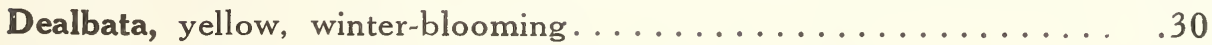

Decurrens, free flowering, beautiful foliage.............. . 30

Eburnea................................ . . . 30

Floribunda, winter-blooming, yellowish............... . 30

Longifolia (latifolia), yellow, beautiful pot plant........... 30

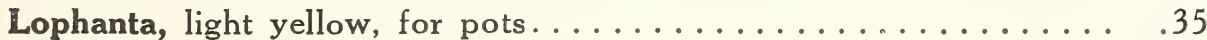

“ Neumanni

Melanoxylon, sweet scented.

\section{ACROCLINIUM (Everlasting)}

Roseum, rose.......................... . $\$ 0.20$

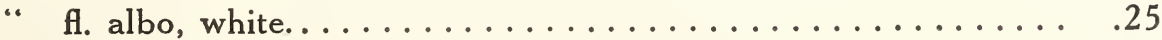

“ fl. pleno, double rose..................... . 25

\section{ALYSSUM}

Little Gem (Tom Thumb) dwarf and compact. . . . . . . . . . . \$0.10

Little Dorrit, white, a gem for edgings and beds. . . . . . . . . . . . 40

Thorburns Bouquet, white, very dwarf, upright growth . . . . . . . .50

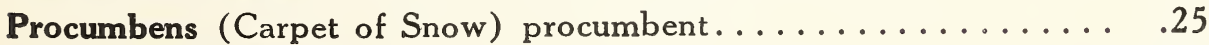


Mexicanum pumilum, Little Blue Star, light blue........... $\$ 1$ oz.

Little Dorrit, azure blue............. . . 25

Princess Victoria Louise, light blue with white centre .30

Blue Ball, clear blue, new............ . 75

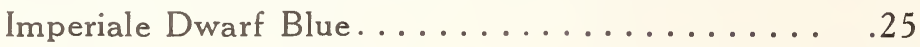

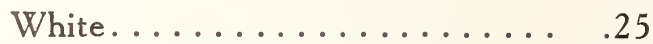

Swanley Blue, dwarf, large flowered....... .50

Dwarf blue.

White

Luteum, bright yellow.
$1 / 2$ oz.

$\$ 1.90$

.50

.60

1.50

.50

.50

1.00

.50

.50

1.00
1 oz.

$\$ 3.50$

1.00

1.00

2.80

1.00

1.00

1.75

1.00

1.00

1.75

Majus (tall) 3 feet high

\section{ANTIRRHINUM (Snapdragon)}

Brilliant, scarlet, yellow and white...........\$0.40

Crescia, dark scarlet...................... 4.40

Firefly, scarlet and yellow, white throated ........... 4.40

Luteum, pure yellow...................... . . . . . . . . . . .

Roseum, rose.......................... 4.40

Delila, carmine, yellow and white............... 40

Vividly Scarlet............................ 440

Papilionaceum, blood red, white throated............. . . . . . . . . . . .

Ghestia, dark blood red................... 4.40

Finest $\operatorname{mixed} \ldots \ldots \ldots \ldots \ldots \ldots \ldots \ldots \ldots \ldots \ldots . \ldots \ldots \ldots$

$\$ 0.80$

Majus grandiflorum (tall, large flowered), 3 feet high

Rosea, rose.......................... 45

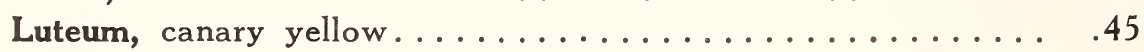

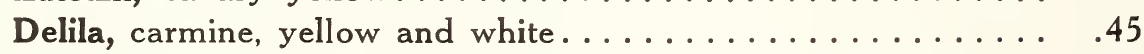

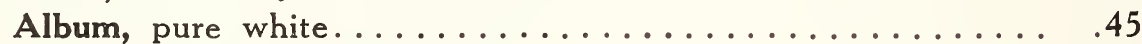

fl. pleno, double white................ 70

Yellow and Orange....................... 4.45

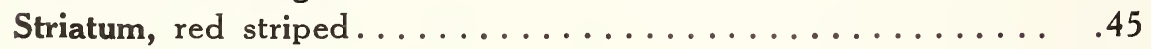

Delicate Rose............................... .45

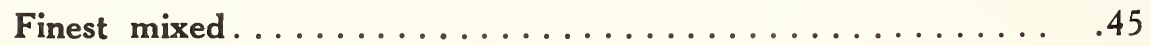

Maximum (The largest flowered Snapdragon), 3 feet high

Snowflake, pure snow-white................. 70

Maralda, coppery-carmine.................. 70

Cinnabar Scarlet, fiery . . . . . . . . . . . . . . . . . . . . 70

Golden Queen, canary yellow with lilac rose........... 70

Ruby, pure ruby red.................... 70

The Rose, pure rose. . . . . . . . . . . . . . . . . . . 70

Nigrescens, nearly black. . . . . . . . . . . . . . . . 70

Purple King, dark purple................... . . 70

Fire King, orange scarlet, white throated . . . . . . . . . 70

Finest mixed 
Antirrhinum Majus Dwarf (continued)

Golden Queen, yellow

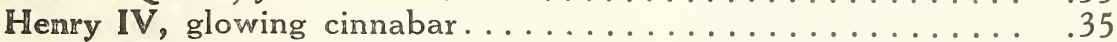

Defiance, fiery scarlet. . . . . . . . . . . . . . . . . . . 35

Fire King, orange scarlet, white throated.............. . . . 35

Niobe, dark purple with white. . . . . . . . . . . . . . . 35

Firefly, dark purple, white throated ................35

$.70 \quad 1.20$

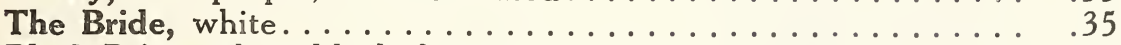

$.70 \quad 1.20$

Black Prince, deep blackish-crimson-purple............. . . . . . . . . . .

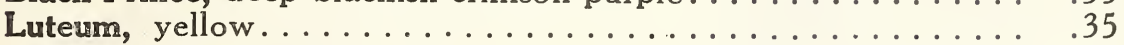

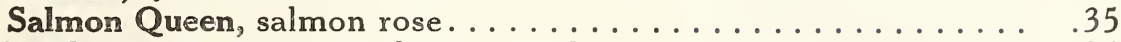

Daphne, carmine rose, white spotted ............... . . . . .

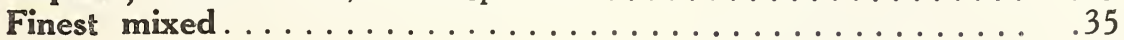

Tom Thumb ( 1 foot high). The best for Pot Plants.

Album, pure white...................... 80

Sulphureum, sulphur yellow . . . . . . . . . . . . . 80

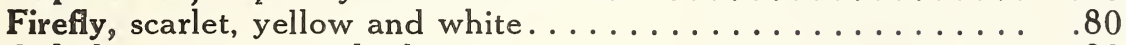

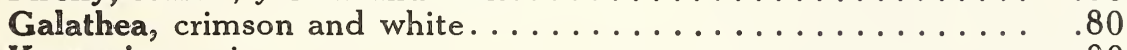

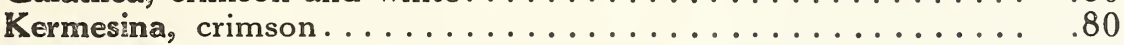

Brilliant Rose. . . . . . . . . . . . . . . . . . . . . 80

Henry IV, glowing cinnabar . . . . . . . . . . . . . 80

Finest mixed .......................... 80

\section{AMARANTUS}

Atropurpureus, dark purple flowers................\$0.15

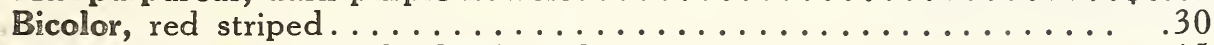

Caudatus (Love Lies Bleeding), red . . . . . . . . . . . . 15

Cruentus (Prince's Feather), deep crimson flowers............ . . . . . . . . .

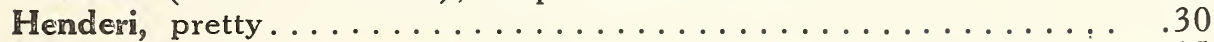

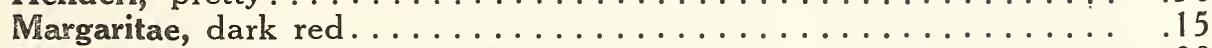

Melancholicus ruber, crimson-leaved .................. . . 30

Monstrosus (Giant. Prince's Feather), deep blood red . . . . . . . . . . . 15

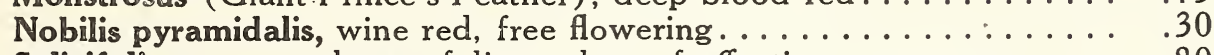

Salicifolius, a very elegant foliage plant of effective appearance. . . . $\quad .80$

\section{ARALIA (May-June delivery)}

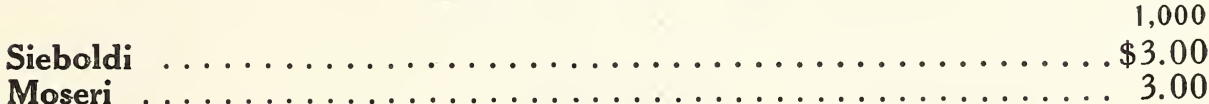

\begin{tabular}{|c|c|c|c|}
\hline \multicolumn{4}{|c|}{ ARDISIA } \\
\hline Crenulata & $\begin{array}{r}1 / 4 \text { oz. } \\
\$ 0.50 \\
.60\end{array}$ & $\begin{array}{r}4 \mathrm{oz} . \\
\$ 1.80 \\
2.20\end{array}$ & $\begin{array}{r}1 \mathrm{lb} . \\
\$ 6.50 \\
8.00\end{array}$ \\
\hline
\end{tabular}




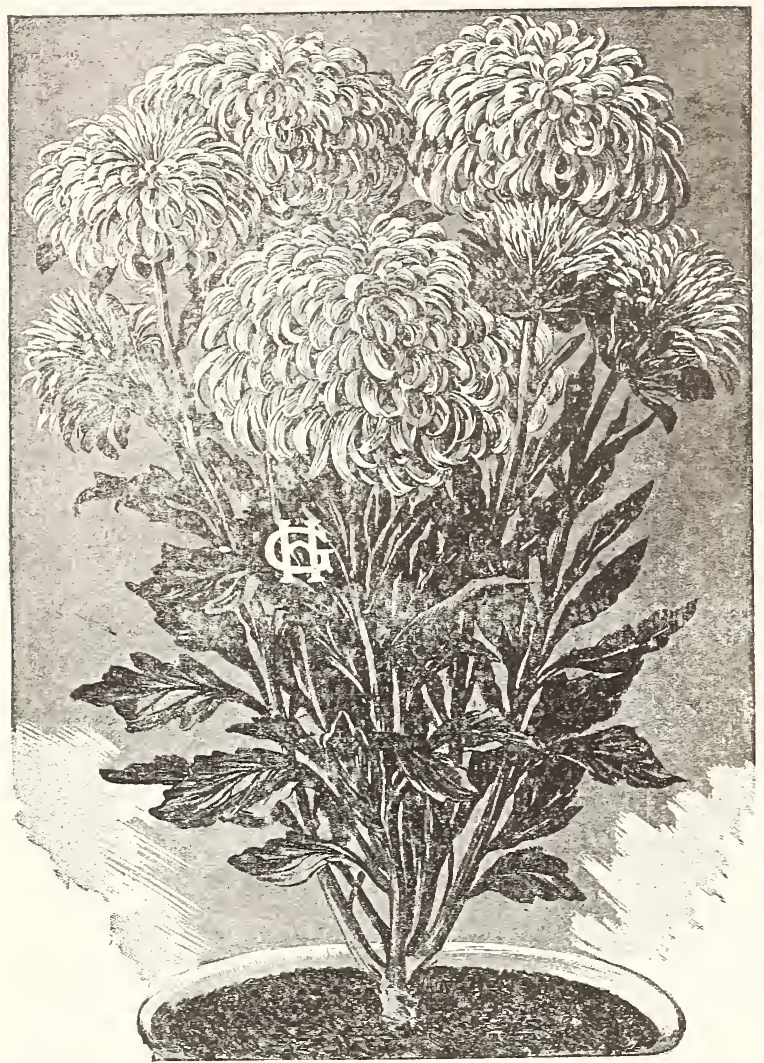

Aster

\section{ASTERS (continued)}

My collection of Asters comprises the most beautiful and perfect varieties in cultivation. All my seed has been produced in the establishment of Ernest Benary in Erfurt, Germany. The product of this grower took the first honors at the International Flower Show at Dresden this year. Purchasers of this seed will feel highly satisfied with the results they will obtain.

Queen of the Dwarfs (Early, very large flowering), 10 inches high.

$\begin{array}{lll}\text { White } & \text { Salmon } & \text { Scarlet } \\ \text { Crimson } & \text { Brown Violet } & \text { Azure Blue } \\ \text { Dark Blue } & \begin{array}{c}\text { Brilliant Rose } \\ \text { Mixture }\end{array} & \text { Light Blue }\end{array}$

$1 / 4$ oz. $\$ 0.80,1 / 2$ oz. $\$ 1.60,1$ oz. $\$ 3.00$

Sada Yakko Aster

Plant about 20 inches high, of pyramidal habit. Flowers delicate $1 / 4$ oz. $\quad 1 / 2$ oz. $1 \mathrm{oz}$. flesh-colored, resembling in shape and size somewhat the large-

flowered Rose Aster, splendid. . . . . . . . . . . . . . \$1.25

\section{Early Flowering Hohenzollern (12 inches high)}

Commencing to bloom the middle of June. Very useful for early cutting.

$\begin{array}{lll}\text { White } & \text { Rose } & \text { Crimson } \\ \text { Scarlet } & \text { Salmon Rose } & \text { Purple Violet } \\ \text { Dark Blue } & \text { Light Blue } & \text { Mixture }\end{array}$

Queen of the Halls (very early-12 inches high)

$\begin{array}{lll}\text { White } & \text { Rose } & \text { Crimson } \\ \text { Scarlet } & \text { Light Blue } & \text { Dark Blue } \\ \text { Salmon } & \text { Cinnabar-Carmine } & \text { Mixture }\end{array}$

\section{Juno Aster}

Plant of globular habit, about 15 inches high, with large sized, fine double, imbricated flowers 4 to 5 inches across. Fine for bedding purposes.

White

White changing to amethyst

White changing to rose

Carmine Rose

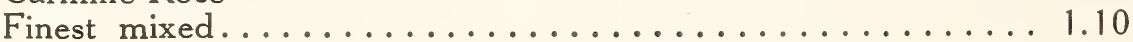


Giant Hohenzollern (30 inches high)

This is considered the grandest Aster ever introduced. Plant tall-growing, and bearing on very long stalks flowers of mammoth size. The flowers being densely double and finely curled.

Unequaled as a cut-flower and highly effective in beds where tall plants are required.

White

Dark Blue

Light Salmon Rose

Lilac

Crown Prince (glowing carmine)

Mixture

Giant Comet (24 inches high). A handsome cut-flower

$\begin{array}{lll}\text { Rose } & \text { Scarlet } & \text { Crimson } \\ \text { Light Blue } & \text { Dark Violet } & \text { Brilliant Rose } \\ \text { Salmon Rose } & \text { The Bride (white, shading to rose) }\end{array}$

Dark Violet

Light Blue

Crimson

Brilliant Rose $\quad 1 / 4 \mathrm{oz}$

$1 / 2$ oz.

2.00

$1 \mathrm{oz}$.

4.00

Ostrich Feathers (about 20 inches high). Cut-flower

A magnificent Aster, freely branching, with long stalked, grace-

ful, fine double flowers of immense size. Finest mixed.......

$.60 \quad 1.10$

American Bush (about 30 inches high)

Long stalked, very large flowers of excellent quality. Very useful for cutting purposes.

Pure White

Scarlet

Lavender

Light Rose

Rose

Dark Blue

Dark Purple

Coppery Red (with crimson centre)

Crimson

Light Blue

Salmon Rose

Cinnabar-Carn

ixture.

Single Flowering (Marguerite) Aster (about 30 inches high)

Very free blooming, beautiful flowers.

$\begin{array}{lll}\text { White } & \text { Purple } & \text { Crimson } \\ \text { Violet } & \text { Dark Blue } & \text { Light Blue } \\ \text { Brick Red } & \text { Fiery Rose } & \text { Purple Violet }\end{array}$

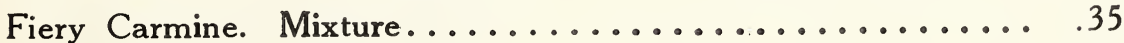

Single Late Flowering (Original China) Aster (30 inches)

White

Violet

Dark Violet
Rose

Purple

Appleblossom
Crimson

Light Lilac

Mixture

Single Japan Aster (about 20 inches high)

Large flowers, excellent for cutting purposes.

White

Rose

Violet

Dark Blue

Crimson

Light Blue

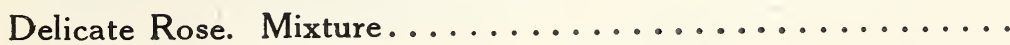




\section{Latest Novelty \\ BEGONIA SEMPERFLORENS}

ZAUBERIN

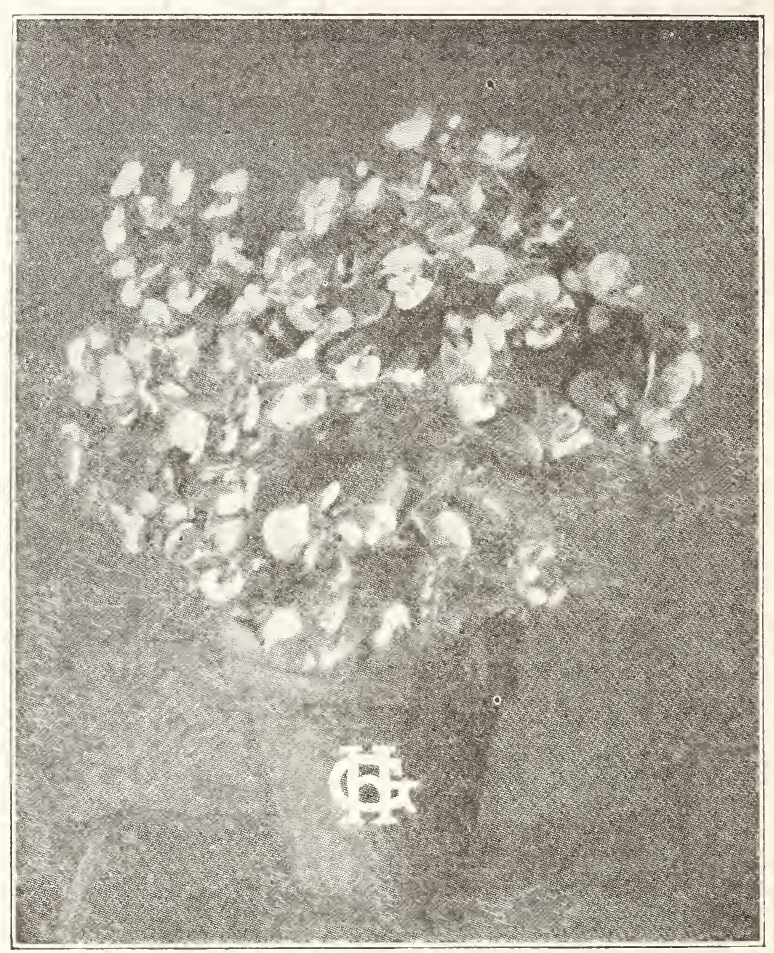

\section{Latest Novelty \\ BEGONIA \\ SEMPERFLORENS}

\section{ZAUBERIN}

This Begonia belongs to the dwarf varieties and is a profuse bloomer, producing an abundance of brilliant pink colored blooms. These plants attain a height of about six inches and grow into a bushy, round-shaped plant.

The extremely large flowers are carried umbrella-like above the dainty reddish foliage which is almost covered up by the flowers. The round and brilliantly pink blooms of about 2 inch diameter remind one of the Lorraine Begonia. In all my experience covering many years I have never seen a variety that gave more promise of conquering the American market.

"Zauberin" is a sport of "Fire Sea" a variety introduced by me three years ago and which was a great success. There is no doubt that "Zauberin" will be an even greater success.

Trade packet, $\$ 2.50$. Five trade packets, $\$ 11.00$. 


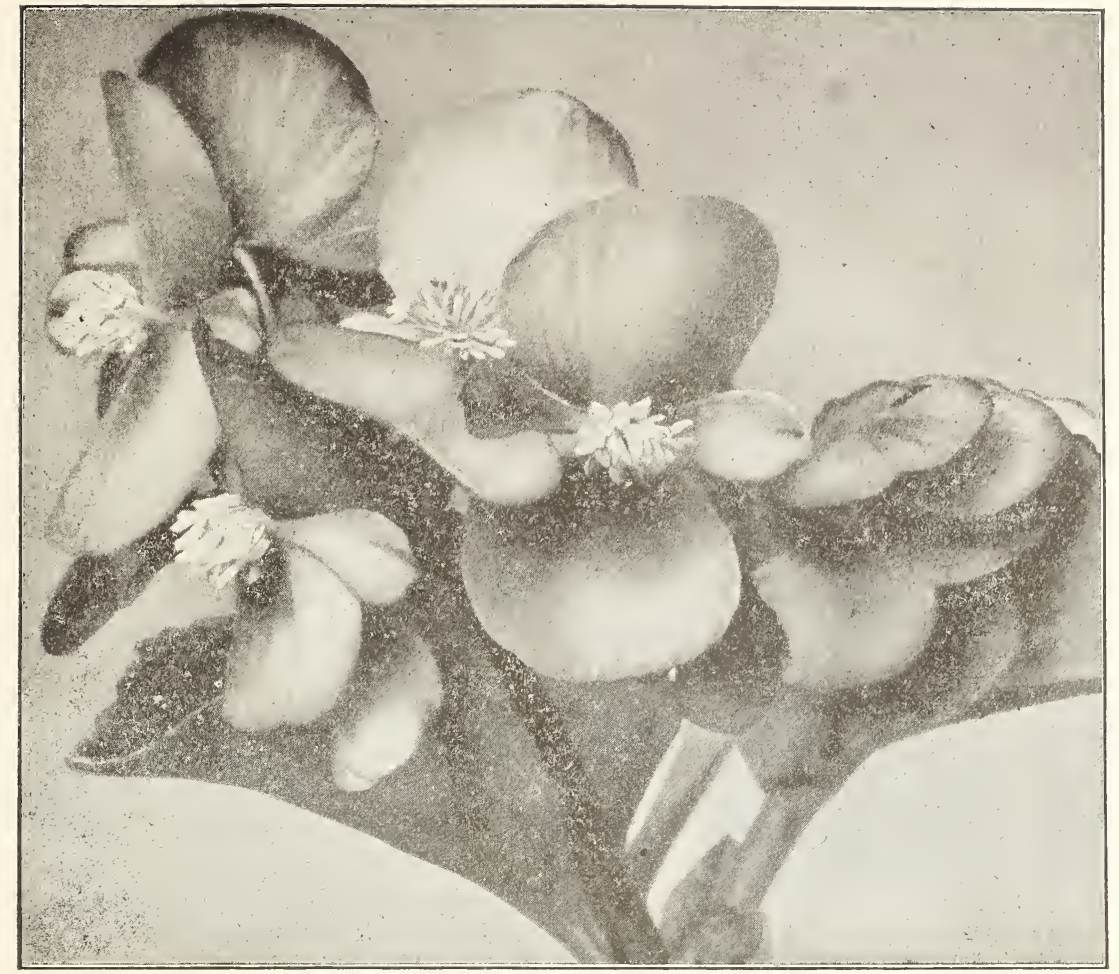

\section{BEGONIAS}

Latest Novelties

\section{BEGONIA SEMPERFLORENS RUNDFUNK (Benary)}

This Begonia is a worthy companion to the Ruhm von Erfurt, introduced a few years ago. These plants will grow to a height of 12 inches, branch out considerable and are profuse bloomers. The blooms grow above the dark green foliage in large clusters. Their color is a clear brilliant red. I wish to state especially that the seed of this Begonia is obtained by crossing certain other varieties and for this reason will always make plants absolutely true to color and size.

Trade packet, $\$ 1.50$. Five trade packets, $\$ 7.00$.

\section{BEGONIA SEMPERFLORENS ROSABELLA (Benary)}

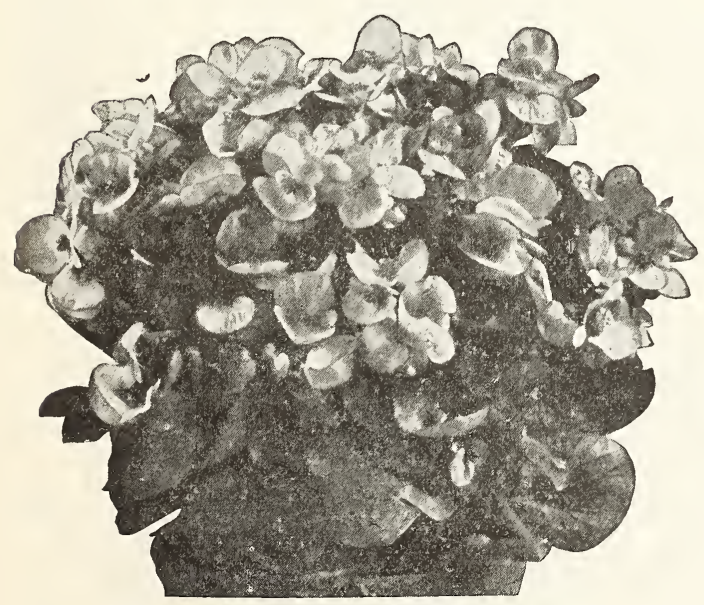

Begonia Semperfi. Rosabella

Among the large assortment of Begonia Semperflorens there has been lacking up to the present time a really low growing pink variety like compacta Rosea, Berta Bofinger, Delicata, Dornroschen. These varieties run very uneven in height and color and their flowers are rather small, After attempts at hybridizing, extending well over twelve years, a variety was obtained that will always; bloom true to name. Rosabella will attain a height of about 6 inches, will branch out and the flowers are of very large size. The color of the: grow very symmetrical. It blooms profusely and blooms is a clear brilliant light pink. Besides border work it will be in demand for pot plants as they are perfect substitutes for Lorraine: Begonias.

Trade packet, $\$ 1.50$. Five trade packets, $\$ 7.00$. 


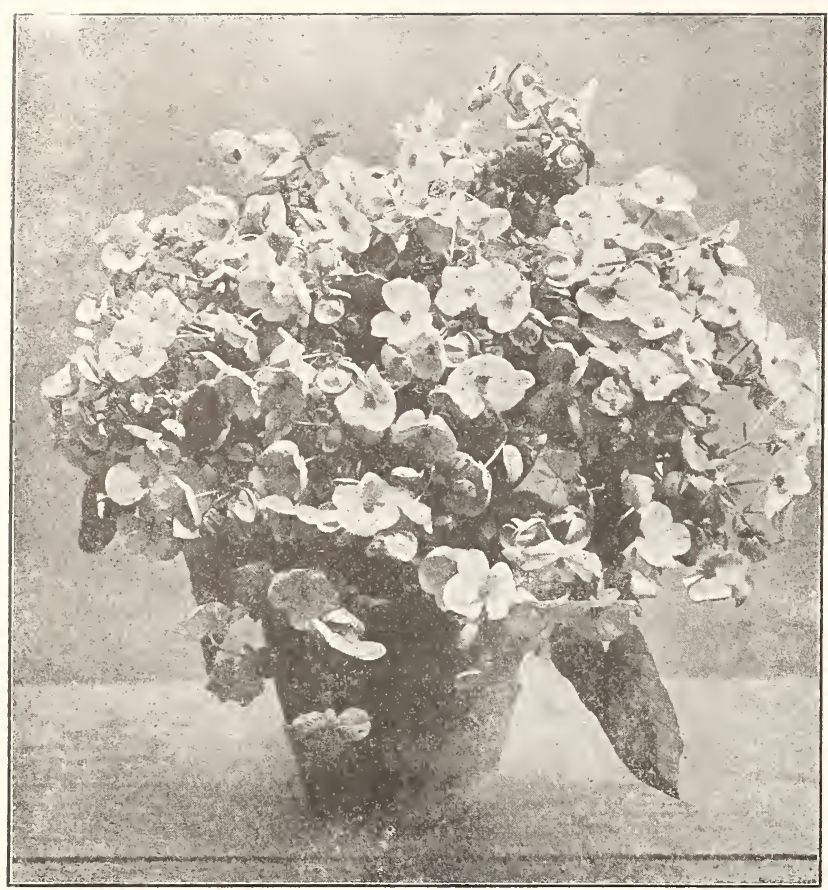

Begonia Semperflorens

\section{BEGONIA SEMPERFLORENS}

I have always made a specialty of $\mathrm{Be}$ gonia seeds, and have always been intent on securing the best the market affords. I wish to call attention to Begonia Glory of Erfurt and Fire Sea. I introduced both of these varieties about three years ago, and they still maintain their position as two of the very best varieties.

The best time for sowing Begonia seeds is from October to January in order to have flowering plants during the entire year. These plants always find willing buyers as they can be used for pot plants as well as for outdonr purposes.

Glory of Erfurt. The most beautiful Begonia on the market. Glowing

1/32 oz. carmine red with silk shine, the blossoms $21 / 2$ inches long and $11 / 4$ inches wide.....................\$1.50

Fire Sea. A very fine and Floriferous Begonia especially good for pot plants. Glowing dark red blossoms................ 1.50

Schwabenstolz. A novelty with especially large umbels of brilliant kermosine color. This Begonia is the earliest blooming one of its kind. The plant is of rich branching growth and reaches a height of about 6 inches.................. 1.50

Schmidt's Ideal. A novelty with a fabulous richness of 2 inches wide blossoms of a dark scarlet color, the blossoms standing 2 inches above the metallic shining foliage.............. 1.50

Liegnitz. Blossoms glowing carmine red and very large......... 1.25

Luminosa. Blossoms dark red, leaves red brown . . . . . . . . . 1.50

Primadonna. Very good winter blooming, pink kermosine....... 1.50

$3.00 \quad 6.00$

Chatelaine Hybrida. Blossoms brilliant rose and extra large . . . . . 1.50 


\section{BEGONIA (continued)}

Queen of the Groups. Flowers 2 inches diameter, dark rose, leaves dark green, brownish red tint and bordered..........\$1.25

$\$ 2.50 \$ 5.00$

Wurttembergia. Carmine-red, richly branched, very good for groups 1.00

$2.00 \quad 4.00$

Flame of Love. Glowing red flowers. . . . . . . . . . . 1.00

$2.00 \quad 4.00$

Mignon. Blossoms glowing salmon............... 1.00

$2.00 \quad 4.00$

Fireball. Blossoms fiery crimson. . . . . . . . . . . . 1.00

$2.00 \quad 4.00$

White Pearl. Large pure white blossoms................ 1.00

$2.00 \quad 4.00$

Marginata. Blossoms white, rose bordered ............. 1.00

$2.00 \quad 4.00$

Albert Martin. Glowing carmine-purple, large flowering . . . . . . . . 1.00

$2.00 \quad 4.00$

Bijou des Iradines. Filled blossoms, very good for groups. . . . . . . .

Rosalinde. Blossoms carmine-red, leaves brown............. 1.50

Apple Blossom........................... 1.50

Fire Dwarf. Glowing scarlet, dark leaves................ 1.00

Blossom Sea. Very rich blooming, fresh green blueish shining leaves $\quad 1.00$

Queen of the Dwarfs........................ 1.00

White Pearl. Large pure white blossoms. . . . . . . . . . . . . . 75

Magic Fire. Pretty carmine-red blossoms. Leaves brown . . . . . . . . $\quad .75$

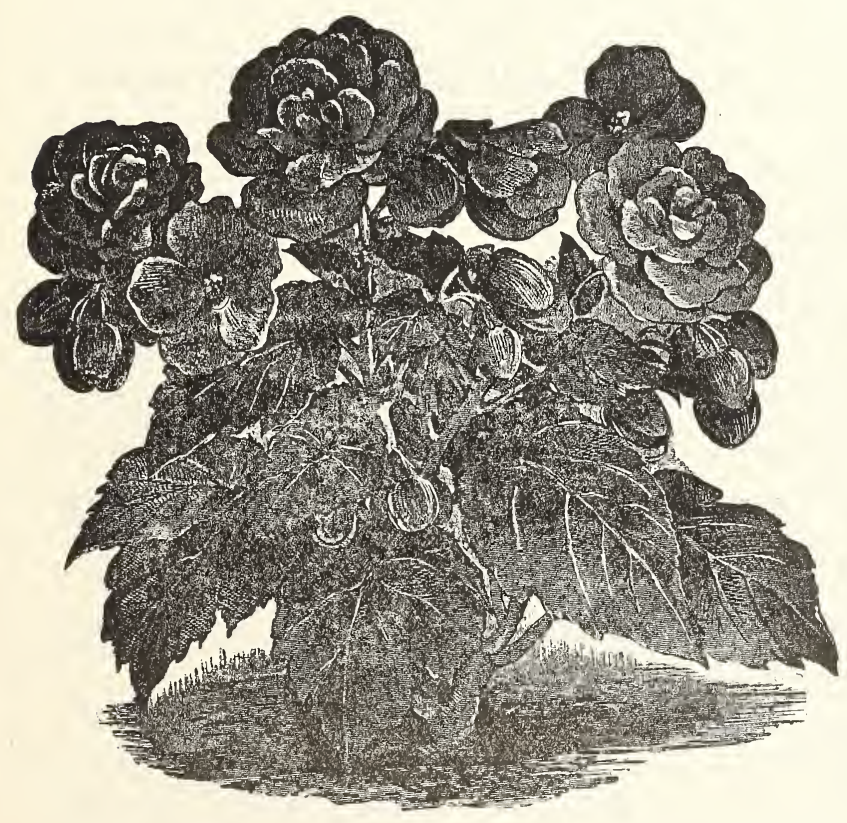

BEGONIA TUBEROUS-ROOTED

Benarys Giant Filled Blossoms mixed ..... Trade packet $\$ 1.00$

Benarys Giant Ruffled Blossoms mixed ...... Trade packet 1.00

Benarys Giant Single Blossoms mixed ......Trade packet 1.00

\section{Benarys Giant Half Filled Blos-} soms, mixed.... Trade packet 1.00

Benarys Double Marbled Blossoms, mixed.... Trade packet 1.00 


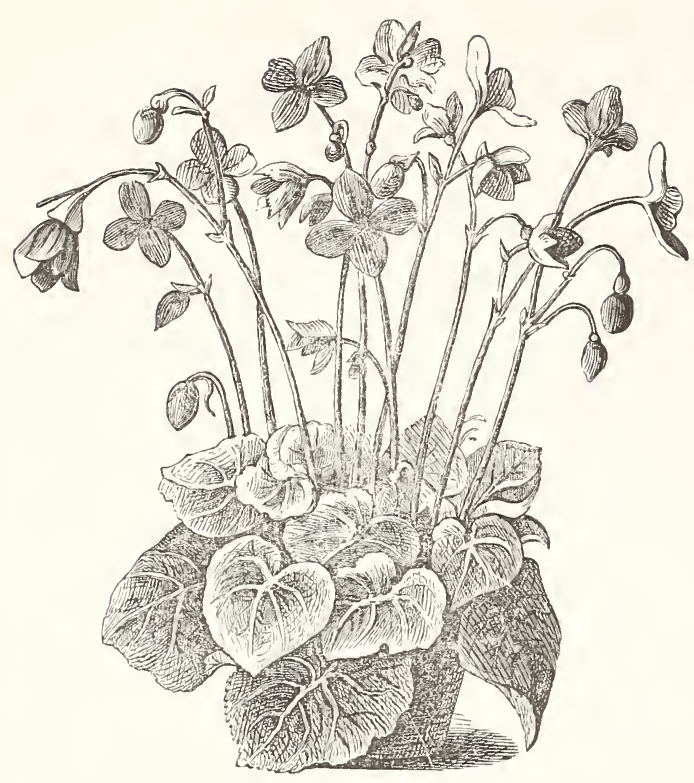

\section{BEGONIA (continued)}

Froebelli. Brilliant-scarlet flowers.

Trade packet. . \$ $\$ 1.50$

Pendula fl. pl. (hanging basket). A Begonia with attractive pending blossoms on delicate stems and of many colors.

Trade packet... 2.00

Corallina. Flowers scarlet, foliage punctuated.

Trade Packet. . .

1.50

\section{Begonia Froebelli}

Improved Double Camellia.

\section{BALSAMS}

White, Crimson, Lilac, Rose Spotted, Violet, Rose Purple, Scarlet Spotted, Scarlet, Mixture....................... \$0.30 \$0.55 \$1.00

BELLIS perennis ff. pl.

(Double Daisy)

Maxima, pink......................... \$1.00

Monstrosa ff. pl. red........................ 1.00

Monstrosa fi. pl. white . . . . . . . . . . . . . . . .

$2.00 \quad 4.00$

$2.00 \quad 4.00$

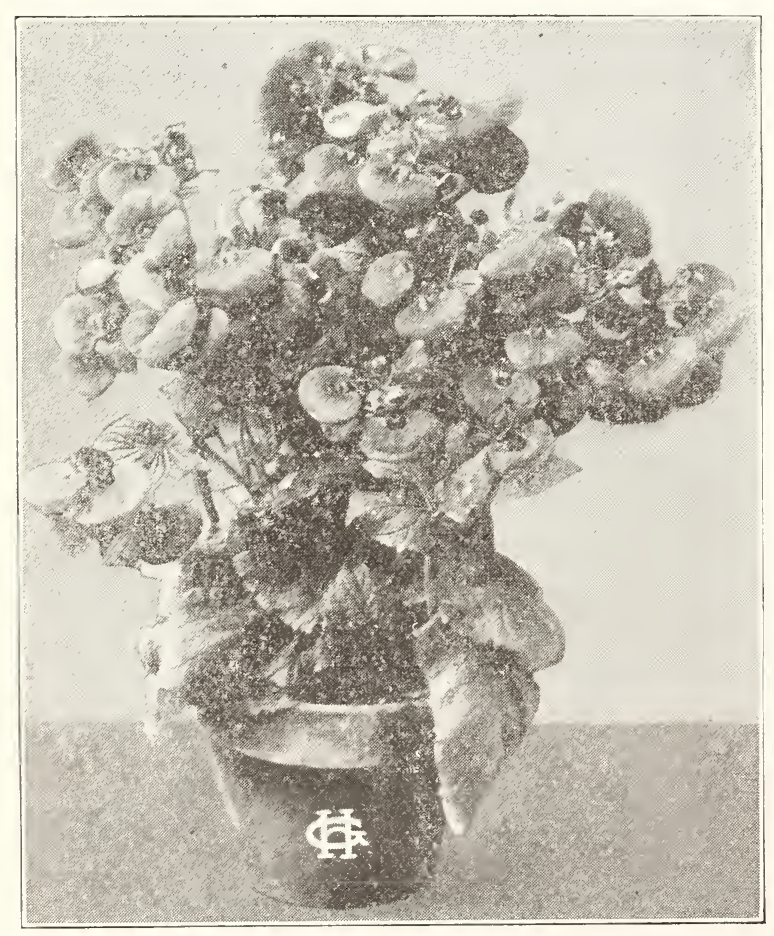

CALCEOLARIA HYBRIDA GRANDIFLORA $1 / 128$ oz. $1 / 64$ oz.

1/32-oz.

Choicest spotted ... $\$ 1.50$

$\$ 3.00$

$\$ 6.00$

Choicest blotched ... 1.50

3.00

6.00

Choicest mixed ..... 1.50

3.00

6.00

Finest dwarf spotted. 2.00

4.00

8.00

Finest dwarf blotched 2.00

4.00

8.00

Finest dwarf mixed. . 2.00

4.00

8.00

Strain Kelway

Choicest mixed . 1.50

3.00

6.00 
Ruggisa aurea (Golden Rain) Small flowers. . . . . . . . . . . . . Trade packet Rugusa major (Triomphe du Nord) Orange-yellow. . . . . . . . . . . . Trade packet

Calceolaria profusa (Clibranii).

Plant growing up to $2 \frac{1}{2}$ feet in height, with innumerable small

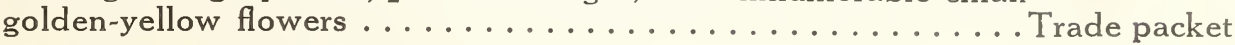

Calceolaria racemosa.

A hardy shrubby freely-branching plant, growing up to a height of 2 feet. Flowers canary-yellow to intense dark brown-red with

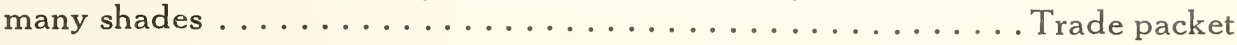

\section{CALENDULA}

Orange King, splendid orange, large flowered . . . . . . . . . $\$$ \$ 50.50

Favorite, light yellow and white.................... . . . . . . . .

Meteor (Striped Pot Marigold), fine double................ . . 25

Lemon King . . . . . . . . . . . . . . . . . . . . . . . . . 25

Orange Ball, best and most filled sort............... .50

Ball's Calendula (Ball's original packages) ............... 1.00

\section{CALLIOPSIS (Coreopsis)}

Bicolor (tinctoria), yellow with brown . . . . . . . . . . . \$0.20

Bicolor marmorata, elegantly marbled and striped............ . 20

Bicolor nigra speciosa, black brown . . . . . . . . . . . . . . . . . 20

Bicolor hybrida semipl, half filled . . . . . . . . . . . . . . . . 20

Bicolor nana, (dwarf), yellow with brown............. 15

Bicolor nana, marmorata, yellow, brown spotted............ . 15

Bicolor nana, purpurea, purple.................. 15

Bicolor nama, sanguimea, (Crimson King)............... 15

Bicolor nana, radiata, brown..................... . 25

Bicolor nana, radiata, Golden Ray, yellow, with brown......... . 25

Bicolor nana, radiata, tigrina, beautifully tigered, new.......... . . 25

Cardaminifolia, atrosanguinea, dark blood red ............. . . . . .

Cardaminifolia, nana compacta bicolor, pretty............. .25

Cardaminifolia, nana compacta atrosanguinea (dwarf) . . . . . . . . 25

Coronata, maxima, large flowered, varying. . . . . . . . . . . . 25

Coronata Drummondi (basalis picta), golden yellow . . . . . . . . . 25

Coronata hybrida superba, finest new hybrids............. . . . . 25

Coronata hybrida superba, mixed................... 20

\section{CANDYTUFT (Iberis)}

Coronaria (Rocket Candytuft), large flowered.......... . \$0.25

Umbellata (Umbelled Candytuft), purple or lilac............. . 30

Umbellata Rose Cardinal, very effective color . . . . . . . . . . . . . . . . . . . . . . .

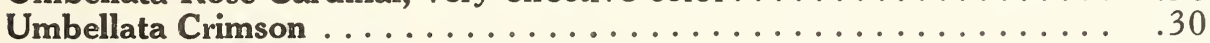

Umbellata, finest mixed . . . . . . . . . . . . . . . . 30

\section{CANNABIS}

Gigantea

\section{COBAEA}


Heddewigi, finest single mixed . . . . . . . . . . . . $\$ 0.50$

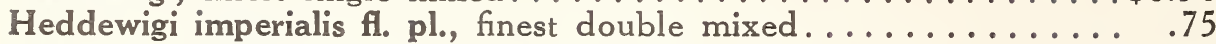

Granat (Grenadin) King of the Blacks, pretty dark shades . . . . . . . . . . . . . . .

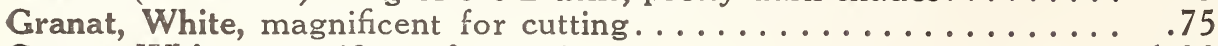

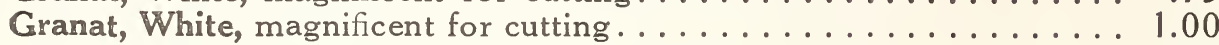

Granat, Rose ............................ 1.00

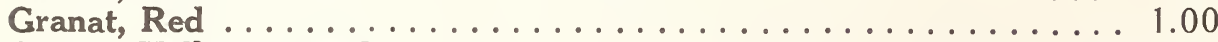

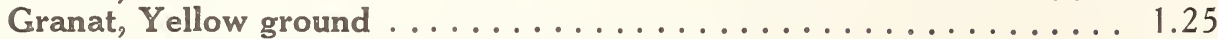

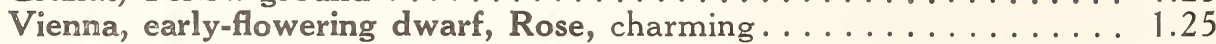

Vienna, early-flowering dwarf, Violet. . . . . . . . . . . . . 1.00

Vienna, early-flowering dwarf, White................. 1.00

Viema, early-flowering dwarf, Tausendschon (salmon) . . . . . . . 1.50

Margarite, finest mixed, will bloom 5-6 months after sowing, free flowering, excellent $f$. cutting. ..................

Plemarius (Garden Pink), finest single mixed . . . . . . . . . . . .

Plumarius, finest double mixed.
$1 / 2$ oz.

1 oz.

$\$ 1.00$

1.50

1.50

1.50

2.00

2.00

2.00

2.50

2.50

2.00

2.00

3.00

1.00

1.50

2.00

$\$ 2.00$

3.00

3.00

3.00

4.00

4.00

4.00

5.00

5.00

4.00

4.00

6.00

2.00

3.00
1.00

\section{CELOSIA}

Cristata nana (Dwarf Cockscomb), finest mixed............ \$0.75

Pyramidalis plumosa aurea, orange-yellow .............. . . . . . . . . . . . .

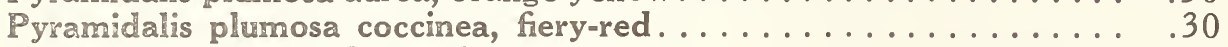

Pyramidalis plumosa kermesina, crimson. . . . . . . . . . . . 30

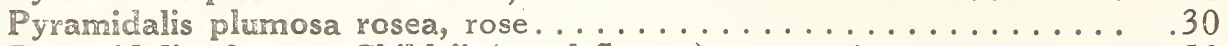

Pyramidalis plumcsa Childsii (wool flower), new, crimson....... . .50

Pyramidalis plumosa Thompconĩ magnifica.............. .75

Pyramidalis plumosa mixed.

$\$ 1.50$

.60

.60

.60

.60

1.00

1.50

.60
$\$ 3.00$

1.20

1.20

1.20

1.20

2.00

3.00

1.00

\section{CENTAUREA}

Moschata coerulea (Blue Sweet Sultan) ............... \$0.25

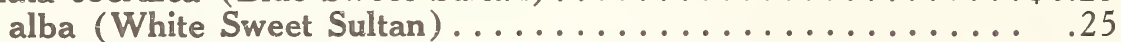

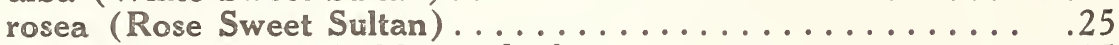

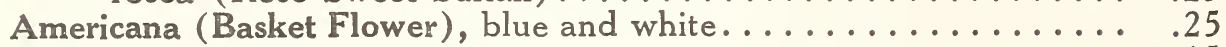

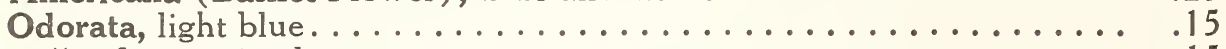

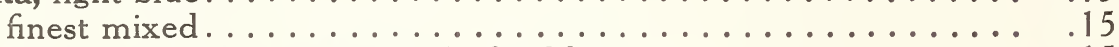

Cyanus azurea (Emperor William) sky blue.............. 15

Argentea Vera (Dusty Miller) . . . . . . . . . . . . . . . .75

\section{CLARKIA}

Elegans ff. pl. Brilliant, salmon scarlet. . . . . . . . . . . \$0.15

carminea, double carmine.............. .15

$\$ 0.30$

rosea, double rose.................. .15

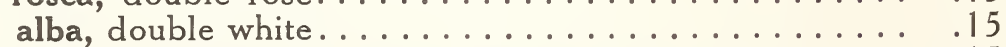

Salmon Queen, extra double salmon rose........ . . 15

Purple King, extra double purple............. .25

Queen Mary, extra double crimson............ . . . . . . . . .

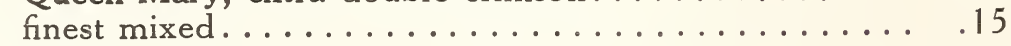

$\$ 0.60$

\section{COSMEA}

Mammoth Lennox, giant flowered, late flowering variety . . . . . . . . \$0.10

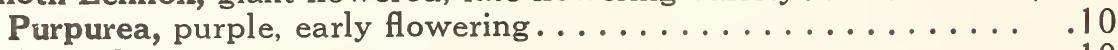

Alba, white, late flowering. .................. 10

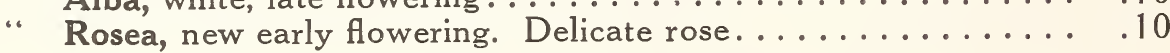




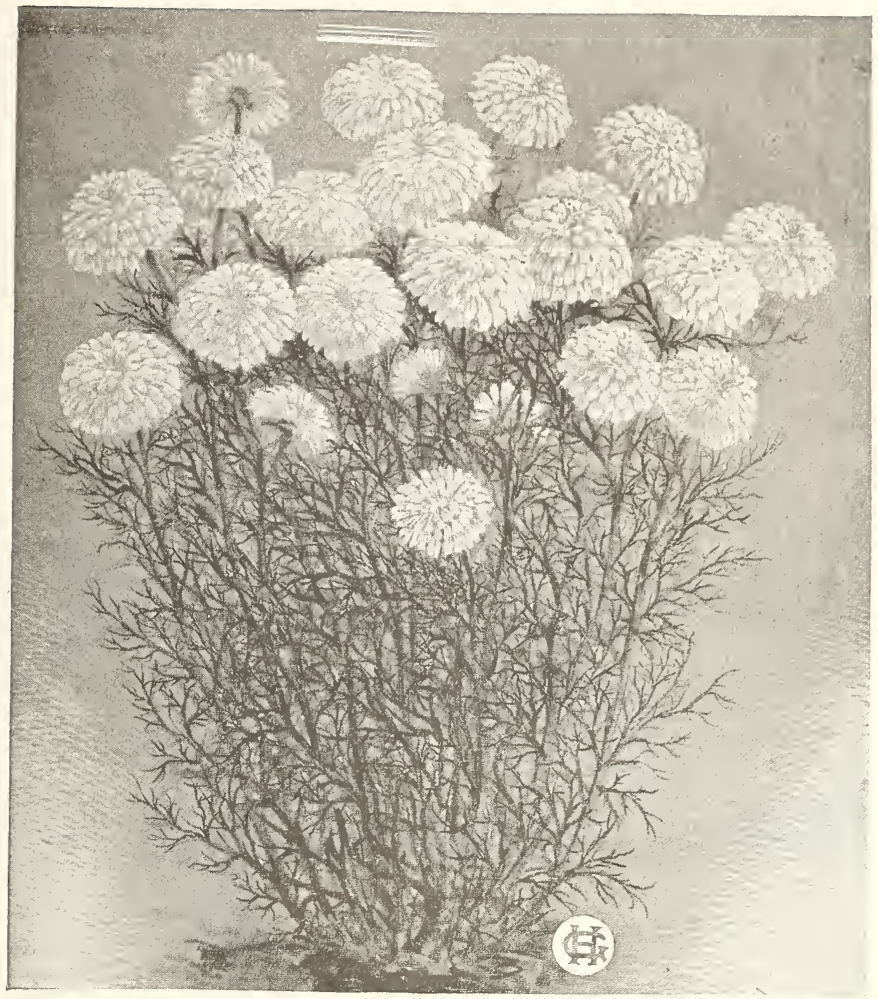

\section{CHRYSANTHEMUM}

Carinatum annulatum (Eclipse), bright orange yellow ...... $1 / 4 \mathrm{oz}$.

" atrococcineum, dark scarlet w. yellow ring.............. . 20

“ Lord Beaconsfield, coppery-bronze................ . . . . . . . . .

“ Dunnetti f. pl. album, extra double white.............. . 20

“ luteum, yellow with dark ring................... . 25

“ Northern Star, large flowered white, with light yellow centre and

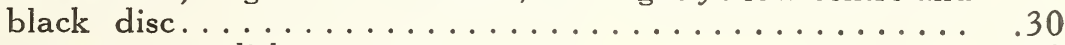

“ purpureum, purplish-crimson.................. . 20

“ radiatum album (Erfurt Star), white, dark disc........... 20

“ " aureum (Golden Wheel), golden-yellow........... . . 20

Coronarium fi. pleno luteam, double yellow. . . . . . . . . . . . . . 30

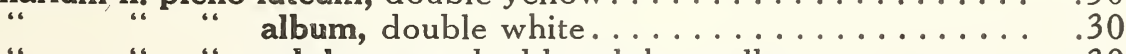

“ “ " sulphureum, double sulphur yellow.......... . 30

Segetum Gloria, light yellow (Morning Star) . . . . . . . . . . . . . 25

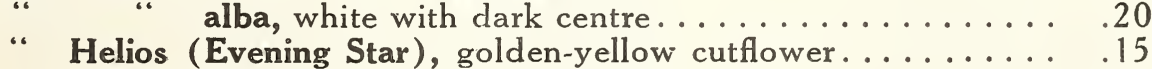

“ Golden Star, golden-yellow, free flowering. . . . . . . . . . . . 15

“ Eastern Star, light yellow, dark centre... . . . . . . . . . . . . 15

Frutescens Comtesse de Chambord (Paris Daisy), white........ . .40

$1 / 2$ oz.

$\$ 0.30$

.40

.50

.40

.50

.60

.40

.40

.40

.60

.60

.60

.50

.40

.30

.30

.30

.75
$1 \mathrm{oz}$. $\$ 0.60$ .80

1.00

1.20

.80

.80

.80

1.20

1.20

1.20

1.00

.80

.60

.60

.60

1.50

\section{COLEUS hybridus}

Laciniatus, laciniated and fringed leaved, finest mixed........ $\$ 1.00$

Macrophyllus, large leaves, finest mixed ............... 1.25

Mixture

.25 


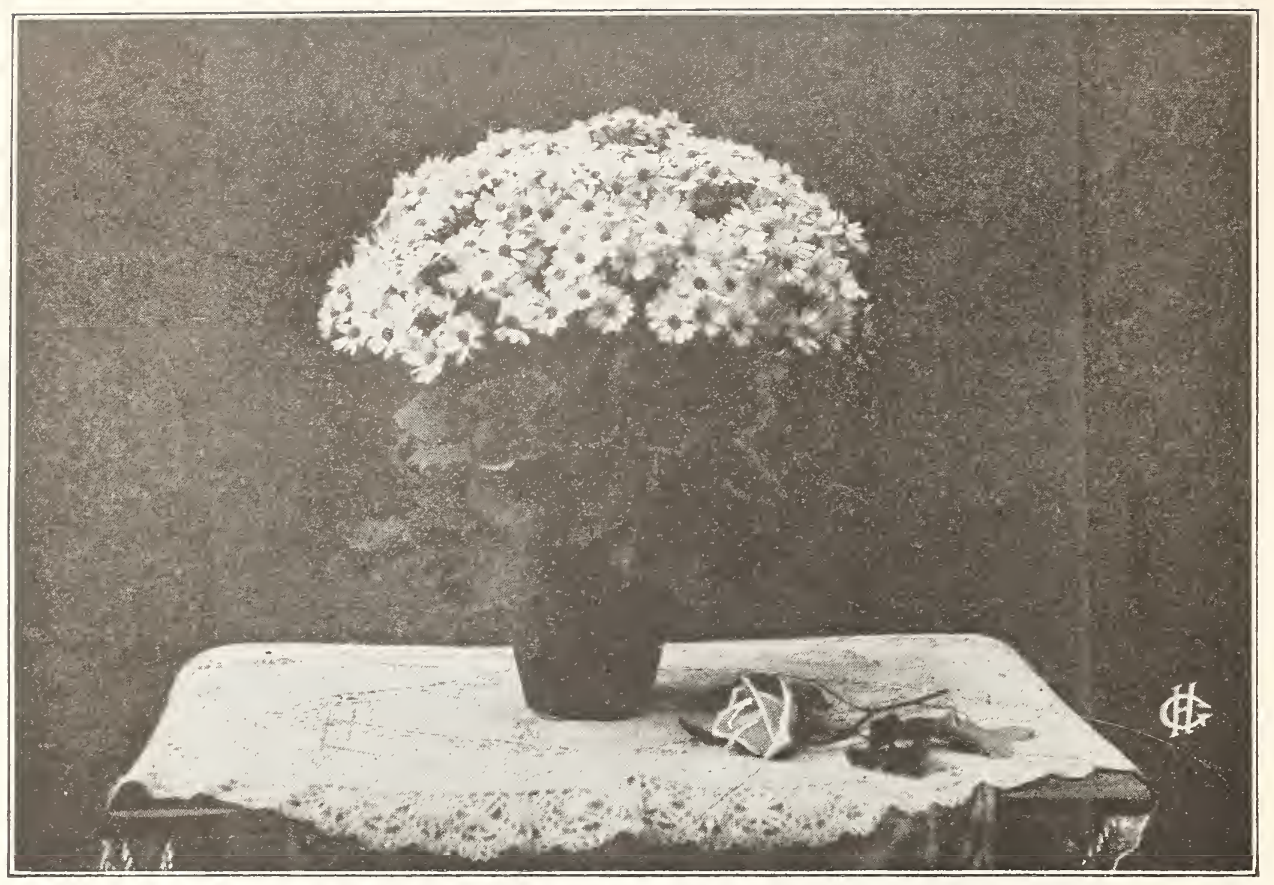

\section{CINERARIA MULTIFLORA NANA}

For commercial purposes the Cineraria Multiflora Nana ranks among the best. The blossoms are about an inch in diameter, and extend in large dense umbels above the delicate foliage. The plant grows very compact and for this reason requires much less space than other Cineraria plants.

The plant is not affected by rot of some of its leaves nor by damage done by snails because these leaves are replaced by new shoots without deforming the plant.

Cineraria Multiflora Nana plants reach

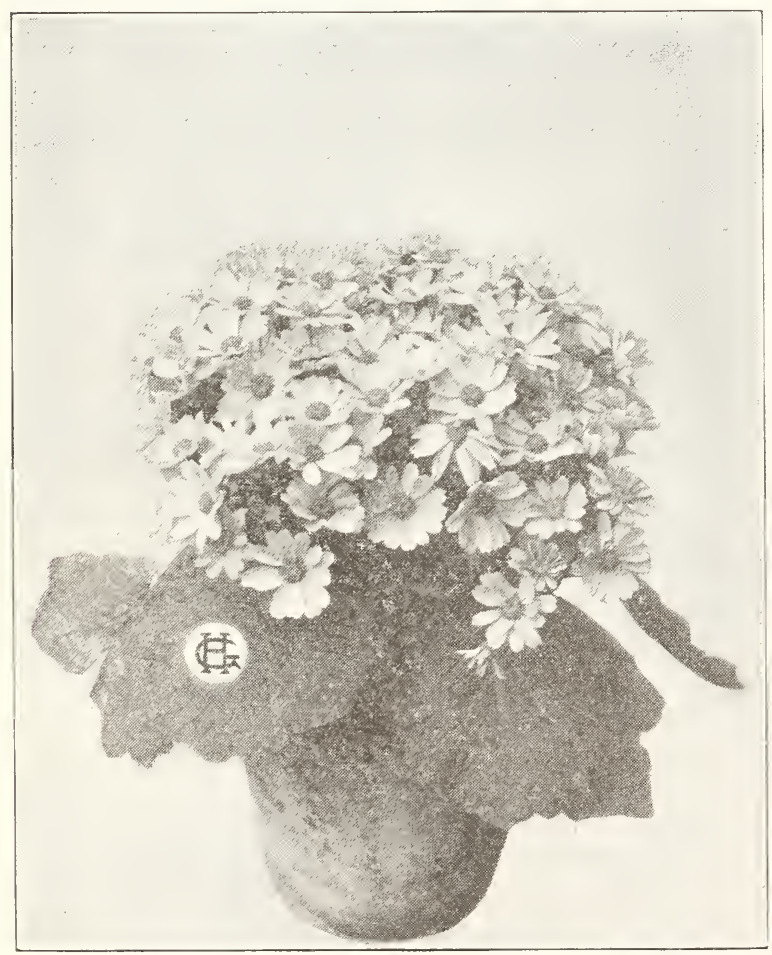
a height of ten inches, and are considered one of the best pot plants in the market today.

If sown early enough they will bloom for Chirstmas, but the plants will be at their best between March and May.

\section{CINERARIA HYBRIDA GRANDIFLORA MAXIMA}

This plant attains a medium height. The large flowers in ten to twelve-inch umbels extend well over the foliage. The leaves are larger than those of Multiflora Nana, and for this reason Maxima is a more decorative plant.

It finds a very ready market for Mothers' Day sales. 
Multiflora Nana (Dwarf)

1/128 oz. 1/64 oz. 1/32 oz.

Rosea

Coerulea

Lilac

Azurea

Rose Kermesina

Kermesina,

Dark Blue

Reddish Dark Blue, Mixture....... \$1.50

$\$ 3.00$

$\$ 6.00$

Hybrida Grandiflora Maxima (Half Dwarf)

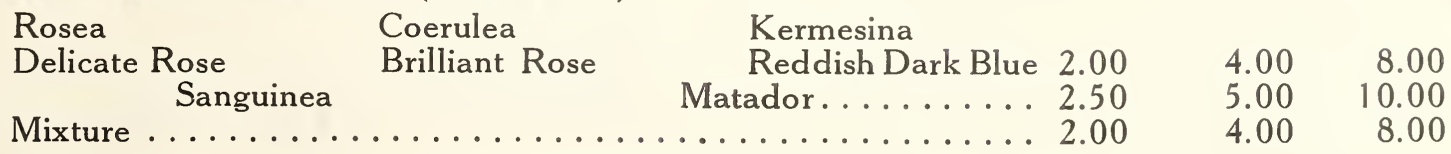

Weissensee Strain Muller (Half Dwarf)

Rosea Red Blue

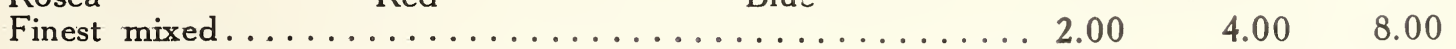

Hybrida Grandiflora (Strain Kelway)

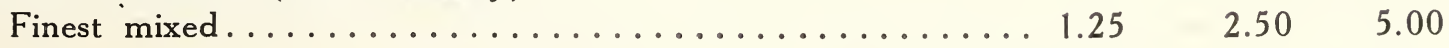

Hybrida Grandiflora Stella. . . . . . . . . . . . . . . . . . . $2.00 \quad 4.00 \quad 8.00$

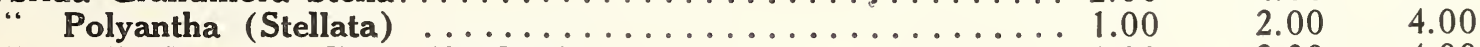

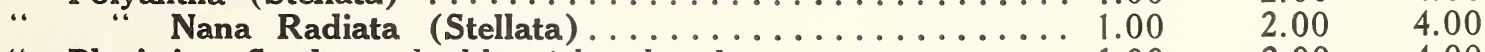

“ Plenissima fi. pleno, double, rich colored............... $1.00 \quad 2.00 \quad 4.00$

Polyantha Radiata, beautifully colored................ .75 $1.50 \quad 3.00$

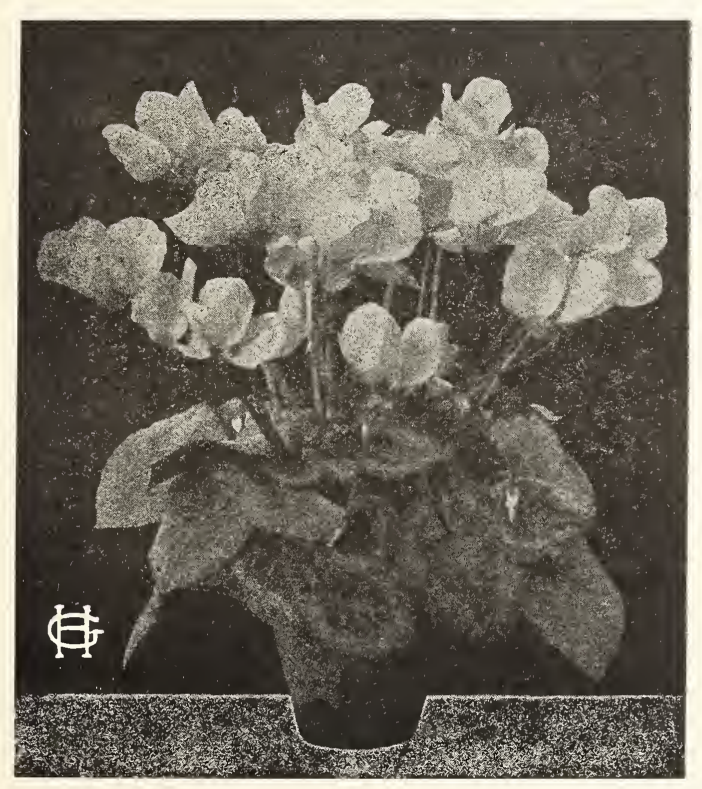

\section{Cyclamen}

The Hundred price applies to all orders up to 500 seeds.

The Thousand price applies to more than 500 seeds.

\section{CYCLAMEN}

(Strain Binnewies)

$\begin{array}{cc}\text { Seeds } & \text { Seeds } \\ 100 & 1000\end{array}$

1/ Dark blood red.... \$1.10\$9.00

2/ Bright red ........ 1.109 .00

3/ Pure rose......... $1.10 \quad 9.00$

4/ Rose of Marienthal. . $1.10 \quad 9.00$

5/ White with carmine eye .............. $1.00 \quad 8.00$

6/ Pure white....... $1.00 \quad 8.00$

7/ Lavender......... 1.2010 .00

8/ Perle von Zehlendorf. $1.40 \quad 12.00$

9/ Rose von Zehlendorf. $1.40 \quad 12.00$

10/ Salmon Scarlet...... $1.80 \quad 15.00$

11/ New Salmon rose improved ........ $1.80 \quad 15.00$

12/ Salmon purple...... $1.80 \quad 15.00$

13/ Rococo .......... 1.8015 .00

14/ Morning red........ 1.8015 .00

$2 \%$ discount on orders for 5-10,000 seeds.

$5 \%$ discount on orders for $11-20,000$ seeds.

$10 \%$ discount on orders for 21-50,000 seeds. 


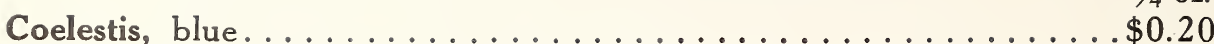

\section{DELPHINIUM (Larkspur)}

Dwarf Hyacinth-flowered ( 2 to 3 feet high)

$\begin{array}{ll}\text { White } & \text { Bright red } \\ \text { Dark blue } & \text { Light Blue } \\ \text { Azure Blue } & \text { Cacao color }\end{array}$

Appleblossom

Brilliant Pink

Carmine, $\quad 1 / 2$ oz.

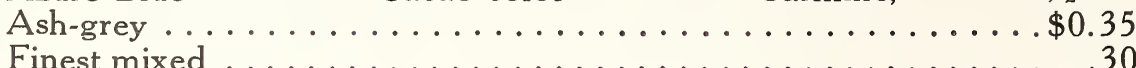

1 oz.

$\$ 0.60$

.55

4 oz.

$\$ 2.20$

2.00

Giant Hyacinth-flowered ( 3 to 4 feet high) early, excellent f. groups
White
Indigo Blue
Light Violet
Rose
Bright Red
Violet
White with blue sheen
Red Grey

Finest mixed

Tall-Filled ( 3 to 4 feet high)

White

Dark Blue

Finest mixed
Crimson
Light Blue
Peach Blossom
Carmine

Bright Red

Apple Blossom

Stock-flowered ( 3 to 4 feet high)

$\begin{array}{ll}\text { White } & \text { Lilac } \\ \text { Salmon } & \text { Dark Blue } \\ \text { Deep Rose } & \text { Tricolor }\end{array}$

Finest mixed

\section{Dark Blue
Tricolor}

.......

\author{
Violet \\ Light Blue \\ White (blue striped) .55
}

\section{DRACAENA}

Indivisa, long narrow, green leaved foliage............ $\$ 0.40$

Australis . . . ........................... $\$ \$^{1 / 4} 0^{\text {oz. }} 50$

Veitchii

\section{DIANTHUS (see Carnations)}

\section{ESCHOLTZIA (California Poppy)}

Rose Cardinal

Rose White

Rose Orange

$\$ 0.10$ 


\section{GAILLARDIA (Blanket Flower)}

Picta Lorenziana bicolor, double brown-red and yellow . . . . . . . $\$ \$ \$$ oz.

“ " rubra, double red, magnificent................ . 20

$1 / 2$ oz. $\quad 1$ oz.

$\$ 0.35 \$ 0.60$

sulphurea, double yellow, beautiful........... .20

\section{GESNERIA}

1/128 oz. 1/64 oz. 1/32 oz.

Macrantha, flowers large, scarlet, splendid.............\$2.00 \$4.00 \$8.00

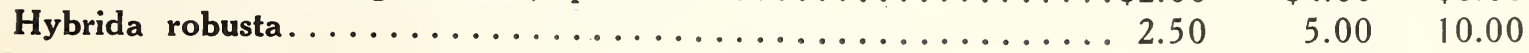

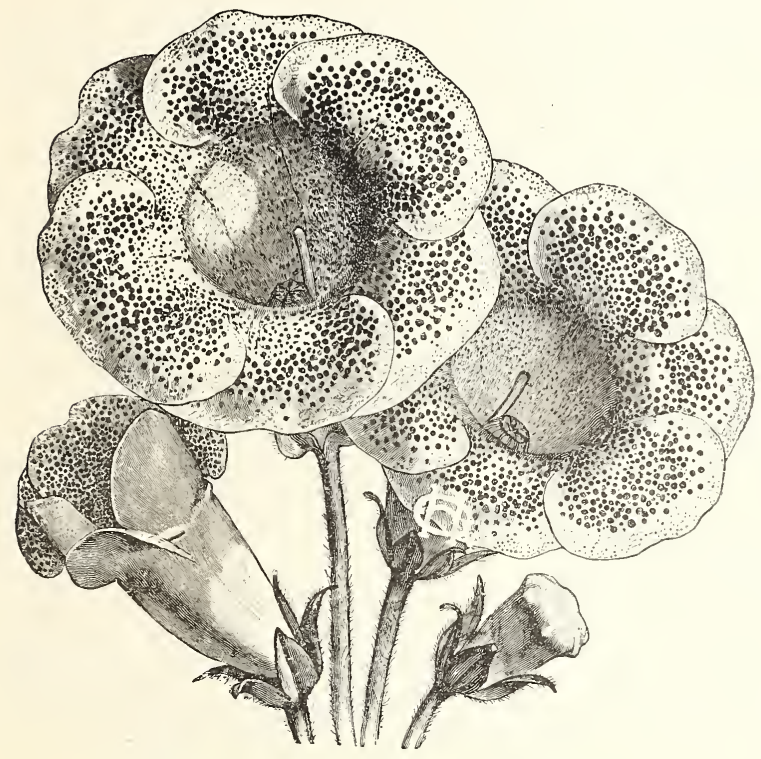

Gloxinia hybrida grandiflora

\section{GLOXINIA hybrida grandiflora}

Trade Packet Finest hybrids mixed.......\$1.00 Gigantea (Giant Gloxinias) ... 1.00 Defiance, flowers upright, large glowing crimson-scarlet.... 1.50

\section{GREVILLEA}

Robusta

1/4 oz. 1/2 oz. 1 oz.

(Silk Oak) . \$0.30 \$0.60 \$1.00

\section{GODETIA}

The Bride, white and red............... \$0. 10

Fire King, glowing purple-carmine................. 10

Queen of Roses, rose on carmine ground . . . . . . . . . . . . . . 15

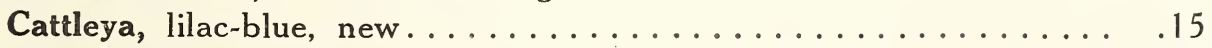

Fairy Queen, white, passing to blush, carmine spots.......... . . 15

Gloriosa, brilliant deep blood-red, magnificent............. . 15

Grandiflora carminea fi. pl., double carmine.............. . . 25

“ rosea fl. pl. double large flowered rose............... 25

“ kermesina fl. pl., double crimson................ . . 25

Carminea aurea, brilliant deep carmine-rose.............. . 20

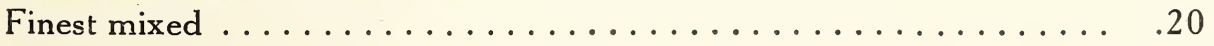

$\begin{array}{rr}1 / 2 \text { oz. } & 1 \text { oz. } \\ \$ 0.20 & \$ 0.40 \\ .20 & .40 \\ .30 & .60 \\ .30 & .60 \\ .30 & .60 \\ .30 & .60 \\ .50 & 1.00 \\ .50 & 1.00 \\ .50 & 1.00 \\ .40 & .80 \\ .40 & .80\end{array}$

\section{GYPSOPHILA}

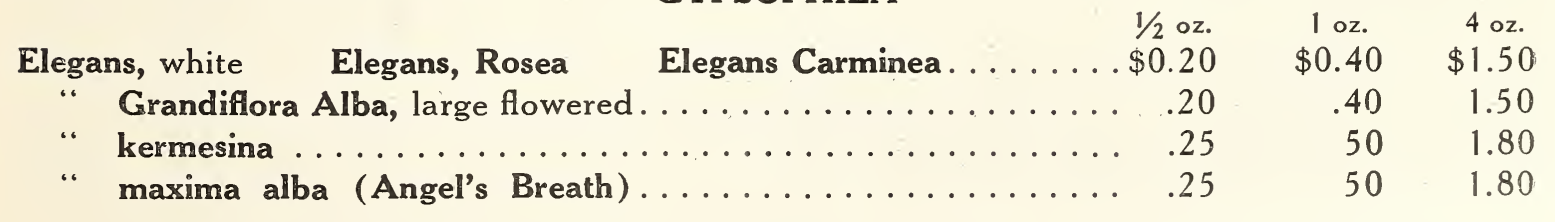


Annuus uniflorus giganteus, flowers of enormous size......... $\$ 0.20$

\section{HELICHRYSUM (Strawflower)}

Monstrosum fi. pleno:

Fireball, fiery-red

Reseum, rose

Luteum, yellow

Album, white

Ferrugineum, dark brown

Finest mixed
Fuscatum, bronze-colored

Salmoneum, salmon-rose

Purpureum, crimson

Sulphureum, light yellow

Rubro-Salmoneum ....... \$0.40

30
$\$ 0.80$ .60
$\$ 3.00$

2.25

\section{HELIOTROPIUM}

Black Prince, dark violet.

1/32 oz.

Queen Margaret, dark blue.

$\$ 0.50$

Anna Turrel, free flowering.

.60

\section{KOCHIA}

Trichophylla (Summer Cypress, Burning Bush)

\section{LANTANA}

Hybrida, mixed

$\$ 1 / 4$ o..

dwarf mixed

$1 / 2$ oz.

$\$ 0.50$

$1 \mathrm{oz}$

.60

\section{LOBELIA}

Erinus compacta Emperor William, bright blue.............. $\$ 0.40$

Crystal Palace (speciosa), deep dark blue......... . 25

“ pumila splendens, dark blue, white eye.......... . .25

“ White Lady, large flowered, pure white.......... . . 25

speciosa Prima Donna, deep dark-red.............. 25

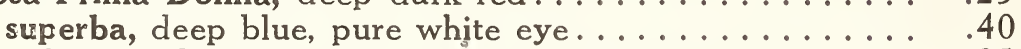

. rich dark blue, purple stems and foliage........... . . . 25

atrocoerulea oculata, dark blue, white eye........... 25

Hybrida pendula Hamburgia, light blue................ .50

Saphir, large deep-blue fls. white eye........ .50

$1 / 16$ oz.

$1 / 8$ oz.

$\$ 0.75$

$\$ 1.50$

.50

1.00

.50

1.00

.50

1.00

.50

1.00

.75

1.50

.50

1.00

.50

1.00

1.00

2.00

1.00

2.00

\section{LUPINUS}

Hybridus, Black

1 oz.

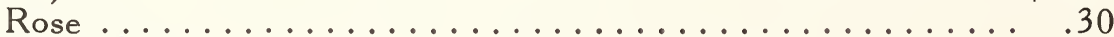

، Rose-Purple

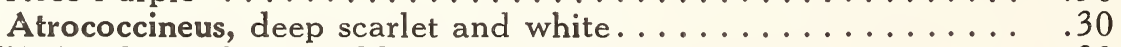

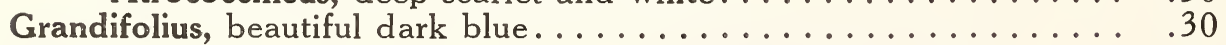

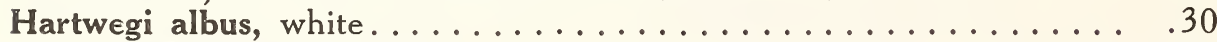

coelestinus, sky-blue....................... . . 30

Dunnetti atroviolaceus, deep violet. ................... . . . . . . . . .

Mutabilis roseus, rose.......................... . . . . . . . . . . . . . .

Sulphureus superbus (Menziesi), beautiful yellow............. . 30

Texensis (subcarnosus), deep rich blue 
Erecta double (African Marigold) 3 feet high

Aurea, golden-yellow . . . . . . . . . . . . . . . \$0.40

Pallida (Pride of the Garden), light yellow............... . . . . . . . .

Sulphurea (Prince Orange), sulphur yellow............ 40

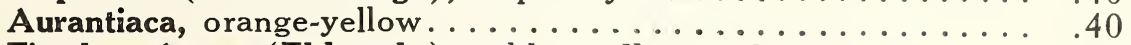

Fistulosa Aurea (Eldorado), golden-yellow quilled . . . . . . . . . .40

Sulphurea, lemon quilled................. .40

1 oz.

4 oz.

Patula double (French Marigold) 3 feet high

$\$ 0.80$

Grandiflora, brown ...................... 440

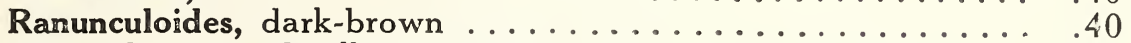

Striata, brown and yellow striped. ............... 40

Tall, dark-brown ...................... 40

Pulchra, yellow, with dark centre................ 40

Patula dwarf double, 1 foot high

Striata, brown and yellow striped ............... 50

Robert Beist, dark red-brown .................. .50

Brown

Gold-Edge, flowers brown-red with golden-yellow edge, very useful for edgings and low beds. . . . .............

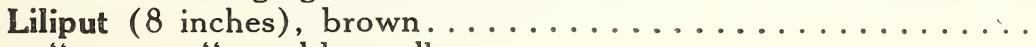

Dwarf mixed golden-yellow

Patula dvarf single ( 8 inches)

Striata, gold-striped ..................... . 20

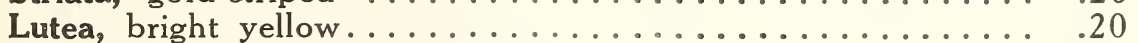

\section{MIGNONETTE (Reseda)}

Bismarck, robust, compact growth, with enormous-sized spikes of large red flowers, extra fine.................... \$0.50

Odorata grandiflora Machet, a dwarf variety, bright-red, finest for pot

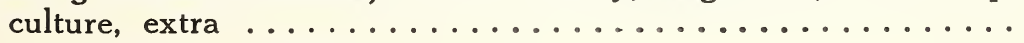

\section{MATRICARIA (Feverfew)}

Eximia double, white quilled . . . . . . . . . . . . . $\$ 0.20$

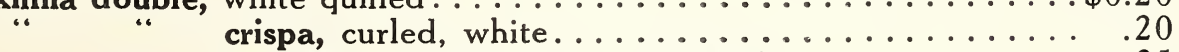

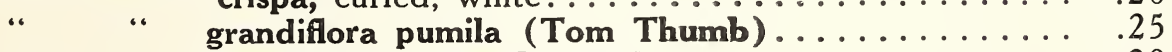

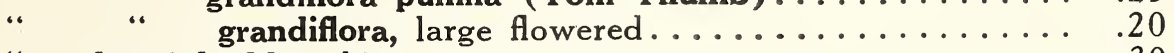

“ dwarf double, white...................... . 30

“ " " Golden Ball, very free flowering.......... 1.00

“ " " Snowball, new, pretty............. 1.25

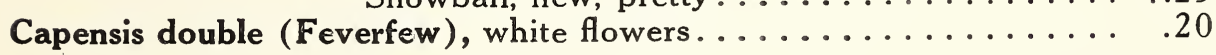

\section{MIMULUS}

Cardinalis, scarlet

\section{MIRABILIS}

(Marvel of Peru or Four O'Clocks)

Jalapa, finest mixed . 
Purpurea (Concolvulus major)
Black Blue
Salmon
Light Blue
Lilac
Carmine
Dark Red
White with Red Gorge

White, Rose Striped, White, Violet Striped.........\$0.20

Imperialis (Japanese), Giant flowering. ................ .30

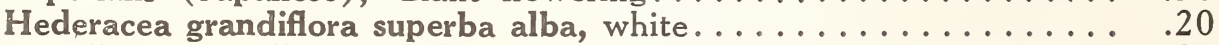

atroviolacea, deep violet..... .20

$\$ 0.40$

.60

.40

.40

$\$ 1.50$

2.25

1.50

1.50

\section{MYOSOTIS (Forget-Me-Not)}

Hybrida Ruth Fischer, large flowers............. $\$ 2.75$

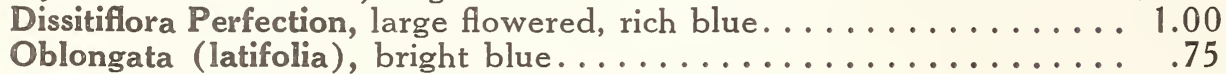

Palustris semperflorens, long-flowering, blue .............. 2.25

$1 / 2$ oz.

2.00

$1 \mathrm{oz}$.

1.50

$\$ 10.00$

4.00

4.25

3.00

8.00

\section{NICOTIANA (Sweet Scented Tobacco Plant)}

Sanderae, rose-carmine, free flowering............... $1 / 2$ oz. $\$ 0.40$

Affinis hybrida, finest mixed.

\section{NIGELLA (Love-in-the-Mist)}

Damascena alba fl. pleno, double, white............ $\$ 0.20$

" nana alba fl. pl., dwarf, double, white.............. . 20

“ f. pl., Miss Jekyll, double, cornflower blue............. . . 20

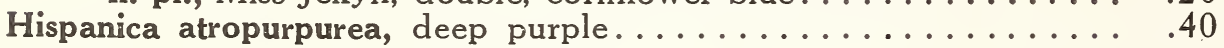

$\begin{array}{rr}1 \text { oz. } & 4 \text { oz. } \\ \$ 0.40 & \$ 1.50 \\ .40 & 1.50 \\ .40 & 1.50 \\ .80 & 3.00\end{array}$

\section{OENOTHERA}

Evening Primrose, sweet scented, yellow............. $\$ 0.20$

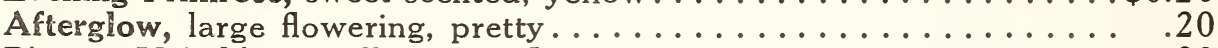

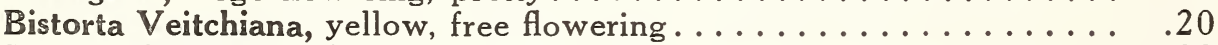

Lamarckiana, large flowering, yellow ................ . . . . . .

Taraxacifolia aurea, golden-yellow, beautiful. . . . . . . . . . . . . 30

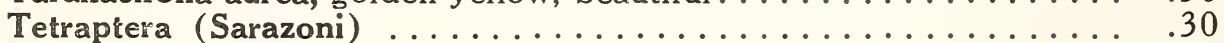

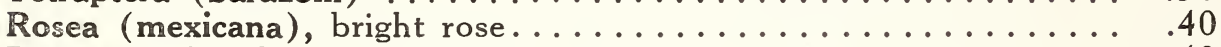

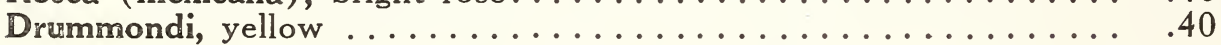

$1 \mathrm{oz} \quad 4 \mathrm{oz}$.

$\$ 0.40 \$ 1.50$

$.40 \quad 1.50$

$.40 \quad 1.50$

$.40 \quad 1.50$

$.60 \quad 2.25$

$.60 \quad 2.25$

$.80 \quad 3.00$

$.80 \quad 3.00$

\section{PAPAVER (Poppy)}

Double Paeony-flowered Poppy ( 3 to 4 feet high)

$\begin{array}{lll}\text { White } & \text { Rose } & \text { Purple } \\ \text { Scarlet } & \text { Salmon } & \text { Ash-Grey } \\ \text { Black } & \text { White and Pink } & \text { Dark Red }\end{array}$

Fiery Cinnabar ...................... \$0.30

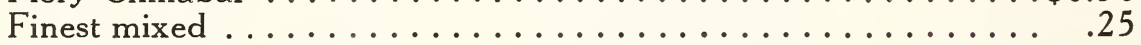

$\$ 0.60 \$ 2.25$

Double Paeony-flowered Poppy ( 2 to 3 feet high)

White

Lilac

Chamois

Brilliant Rose

Rose

Black

Dark Scarlet

Scarlet and White
Purple

Salmon

Coppery Scarlet

Rose-Pink

White, Red Striped, White with Light Purple........... . . 25

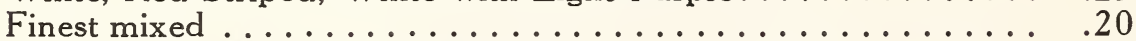

Double Carnation Poppy (somniferum fl. pl.), mixed.......... . . . . . . .

Double Cardinal Poppy (2 to 3 feet high), finest mixed.......... . 30

$\begin{array}{ll}.50 & 1.85 \\ .40 & 1.50 \\ .50 & 1.85 \\ .60 & 2.25\end{array}$




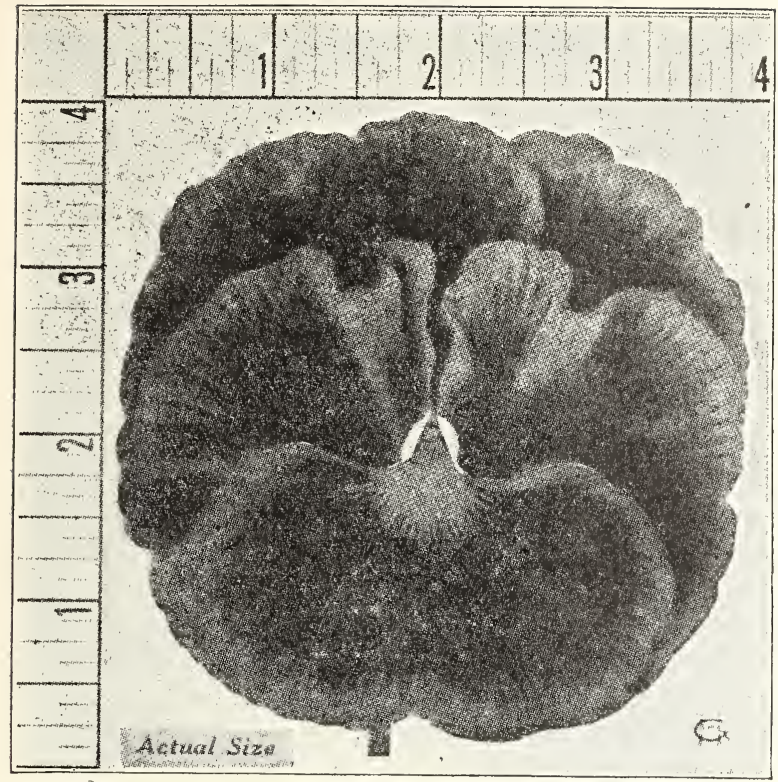

Giant Triumph

\section{PANSIES \\ Giant Triumph}

These new plants distinguish themselves by a strong growth forming compact bushes of round shape and conspicuous large foliage. Upon strong stems the five spotted petals of a completely round form elevate themselves above the foliage with an erect posture in a graceful manner. The extremely broad petals overlap in a manner giving the flower almost the appearance of being twofold. The edge of the petals of most of the flowers is neatly curled without injuring the erect posture of the flower.

The magnificence, the luster and the richness of the colors is very conspicuous, caused by the unique and very dark red and brown tone of colors. Upon each petal of the flower appears prominently the large extremely effective dark colored spot which is oftentimes surrounded by a conspicuously glowing variously colored border.

Goliath, golden-yellow, 5 spotted . . . . . . . . . . . . . \$3.00

Masterpiece (Germania), frilled, spotted, beautiful........ 3.00

Cassier, beautiful blending of colors, dark spotted. . . . . . . . . 3.00

Cardinal, magnificent cardinal-red. . . . . . . . . . . . . . 3.00

Orchidaeflora, pretty . . . . . . . . . . . . . . . . . 2.00

Fire Blotched Bugnot, magnificently colored............ 2.25

Psyche, large flowered, curled, fire-blotched, charming. . . . . . . 1.75

Red Riding Hood, velvety-violet, free flowering.......... 2.50

Adromeda, ruffled, rosy-lavender............. 2.50

Peacock, beautifúl blending of colors. . . . . . . . . . . 2.50

Bridesmaid, white and rose, deep blue spots, charming. . . . . 3.00

Vulcan, very large, brilliant dark-red, 5 black blotches . . . . . . 3.00

Aurora, dark wine-red, dark eye, white border. . . . . . . . 3.00

Light Blue (without eye), dark centre............. 2.50

Auriculaflora, splendid colors. . . . . . . . . . . . 2.50

Yellow with Black Eye, pretty. . . . . . . . . . . . . 3.00

Mercury, velvety black-purple, very large flowers.......... 3.00

Hortensia Red, dark eye................... 3.00

Emperor William, cornflower blue, dark eye. . . . . . . . 3.00

Madame Perret, red marbled shades, pretty........... 2.50

Prince Bismarck, light brown with dark face........... 2.50

Prince Henry, dark blue with dark eye............. 2.50

Dark-Blood-Red, beautiful. . . . . . . . . . . . . . . 3.00

Preciosa, purple violet, whitish ground, dark violet spots..... 3.00

Ruby King, dark red, extra fine................ 3.00

Lord Beaconsfield, purple-violet, running out into white...... 3.00

White with Purple Eye, very beautiful . . . . . . . . . . 3.00 


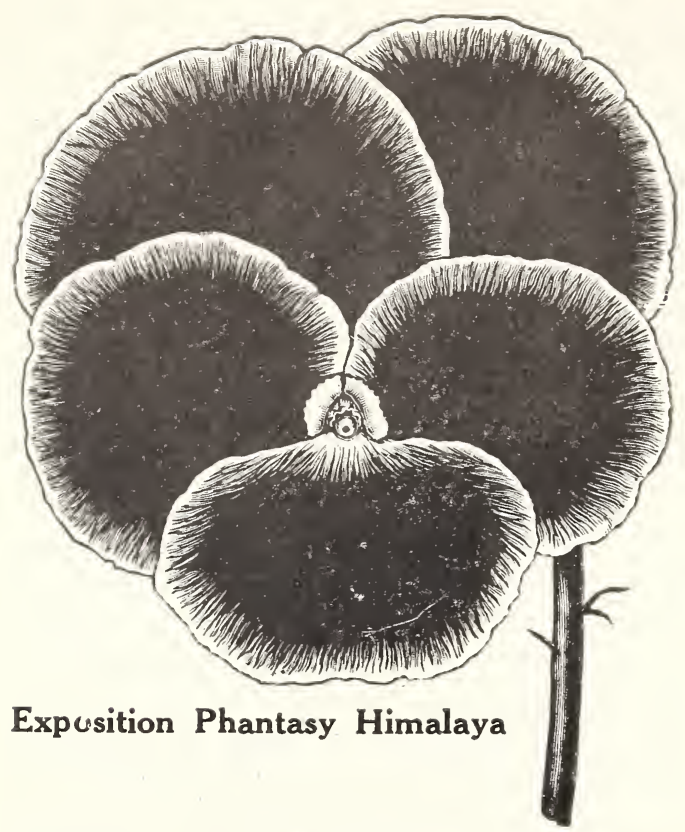

\section{EXPOSITION PHANTASY HIMALAYA (Ice Pansies)}

Are receiving the highest awards. They are carrying off the world's record prizes. Ceaseless labor and experiments have produced these winter-flowering and sweet-smelling plants.

The first flowering sets in at the beginning of October, keeps right on during the whole of the fall until it snows, but they keep on flowering and blossoming under cover of snow, and just as soon as spring melts the ice and the snow and the rays of the sun shine upon them, they again unfold their glorious blossoms as if nothing had happened. The early and late, the almost continually blooming Giant World's Pansies certainly give the world an almost continuously flowering and charming ground cover, combining a late fall as well as an early spring covering.

\section{Himalaya}

\begin{tabular}{|c|c|c|}
\hline $\begin{array}{l}\text { Helios, clear golden-yellow, pretty } \ldots \ldots \ldots \ldots \ldots \ldots \ldots \ldots \ldots \ldots \text {. } \ldots \ldots \text { oz. } \\
\text { Jupiter, lower leaves purple violet, blue above and running out }\end{array}$ & $\frac{1}{\$ 2}$ oz. & $\begin{array}{l}1 \text { oz. } \\
\$ 6.00\end{array}$ \\
\hline 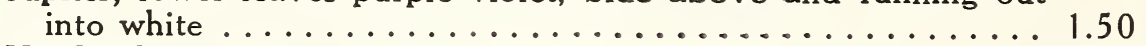 & 3.00 & 6.00 \\
\hline Northpole, pure snow white. & 3.00 & .00 \\
\hline of March, velvety sky blue. & 3. & 6.00 \\
\hline ing, silvery-white, with dark bl & 3. & 6.00 \\
\hline , black . . . . . . . . . . . . & 3. & 6.0 \\
\hline 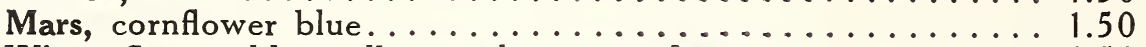 & 3. & 6.0 \\
\hline Winter-Sun, golden-yellow with eye markings.......... & 3.00 & 6.00 \\
\hline Queen of the Heaven, sky-blue....... & 3.00 & 6.00 \\
\hline 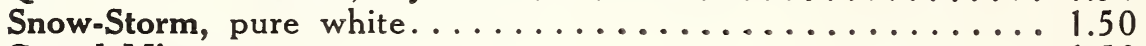 & & 6.00 \\
\hline $\begin{array}{r}\text { Grand Mixture } \ldots \ldots \ldots \ldots \ldots \ldots \ldots \ldots \ldots \ldots \ldots \ldots \ldots \\
4 \text { ounces, } \$ 20.00\end{array}$ & 3. & 6.00 \\
\hline
\end{tabular}

\section{Tricolor Maxima}

Light Blue with eye. . . . . . . . . . . . . . . 2.00

Coquette de Poissy, rosy-light-blue........................ 2.00

Fairy Queen, light blue, white edged............. 2.00

Meteor, fiery brown...................... 2.00

Purple Violet, pretty. . . . . . . . . . . . . . . 2.00

Wallflower Brown, charming . . . . . . . . . . . . . 2.00

Atropurpurea albo-marginata, dark purple, silvery edged. . . . 2.00

Black with Gold Bronzy Face. . . . . . . . . . . . . . . 2.00

Victoria, purple violet with dark eye............... 2.00

Candidissima (alba pura Snow Queen), pretty........... 2.00

Fire King, golden-yellow, running into purple-brown . . . . . 2.00

Gold-margined, dark brown, with yellow edge and eye. . . . . 2.00

Atrosanguinea, deep blood-red. . . . . . . . . . . . . 2.00

Yellow with Eye, pretty . . . . . . . . . . . . . . . . 2.00

Emperor Frederick, dark-red, orange margined......... 2.00

Prince Bismarck, fawn color, dark eye.............. 2.00

Quadricolor (gloriosa perfecta), 4 colored............... 2.00

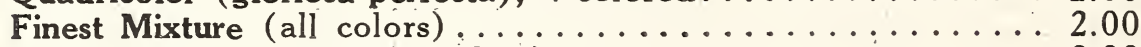

$\begin{array}{ll}4.00 & 8.00 \\ 4.00 & 8.00 \\ 4.00 & 8.00 \\ 4.00 & 8.00 \\ 4.00 & 8.00 \\ 4.00 & 8.00 \\ 4.00 & 8.00 \\ 4.00 & 8.00 \\ 4.00 & 8.00 \\ 4.00 & 8.00 \\ 4.00 & 8.00 \\ 4.00 & 8.00 \\ 4.00 & 8.00 \\ 4.00 & 8.00 \\ 4.00 & 8.00 \\ 4.00 & 8.00 \\ 4.00 & 8.00 \\ 4.00 & 8.00 \\ 4.00 & 8.00 \\ 4.00 & 8.00\end{array}$

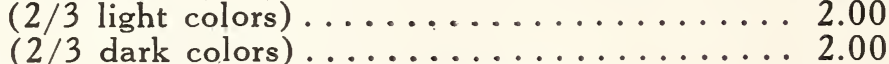

4 ounces, $\$ 28.00$ 
Snowflake, pure white........... $1 / 4$ oz.

Emperor William, cornflower blue.

Golden Yellow with eye.

Pure Yellow without eye.

Violet Blue

White with

Atropurpurea, deep purple violet.

Fire King, yellow ground, marmorized.

Lord Beaconsfield, purple violet, shading to white

\section{PERILLA}

Nankinensis macrophylla compacta, bronze-purple foliage

$\$ 0.15$

$\$ 0.30$

$\$ 0.60$

\section{PHLOX DRUMMONDI}

Grandiflora alba, white.....................\$0.30

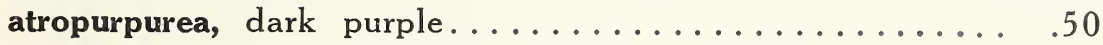

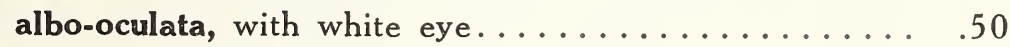

Hortensiaeflora, rosea, rose with dark eye............. .70

Triumph, scarlet ..................... 70

Nana compacta, atropurpurea, deep purplish-crimson......... . 80

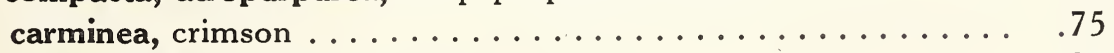

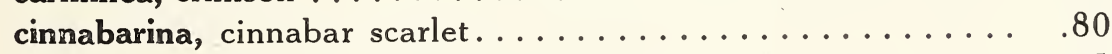

coccinea, scarlet

.75

“ striata, scarlet, white striped............. . 80

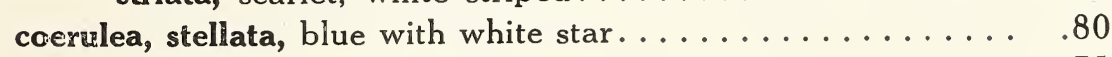

delicata, delicate rose, beautiful . . . . . . . . . . . . . 75

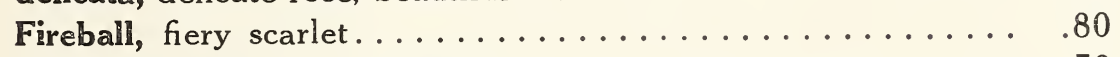




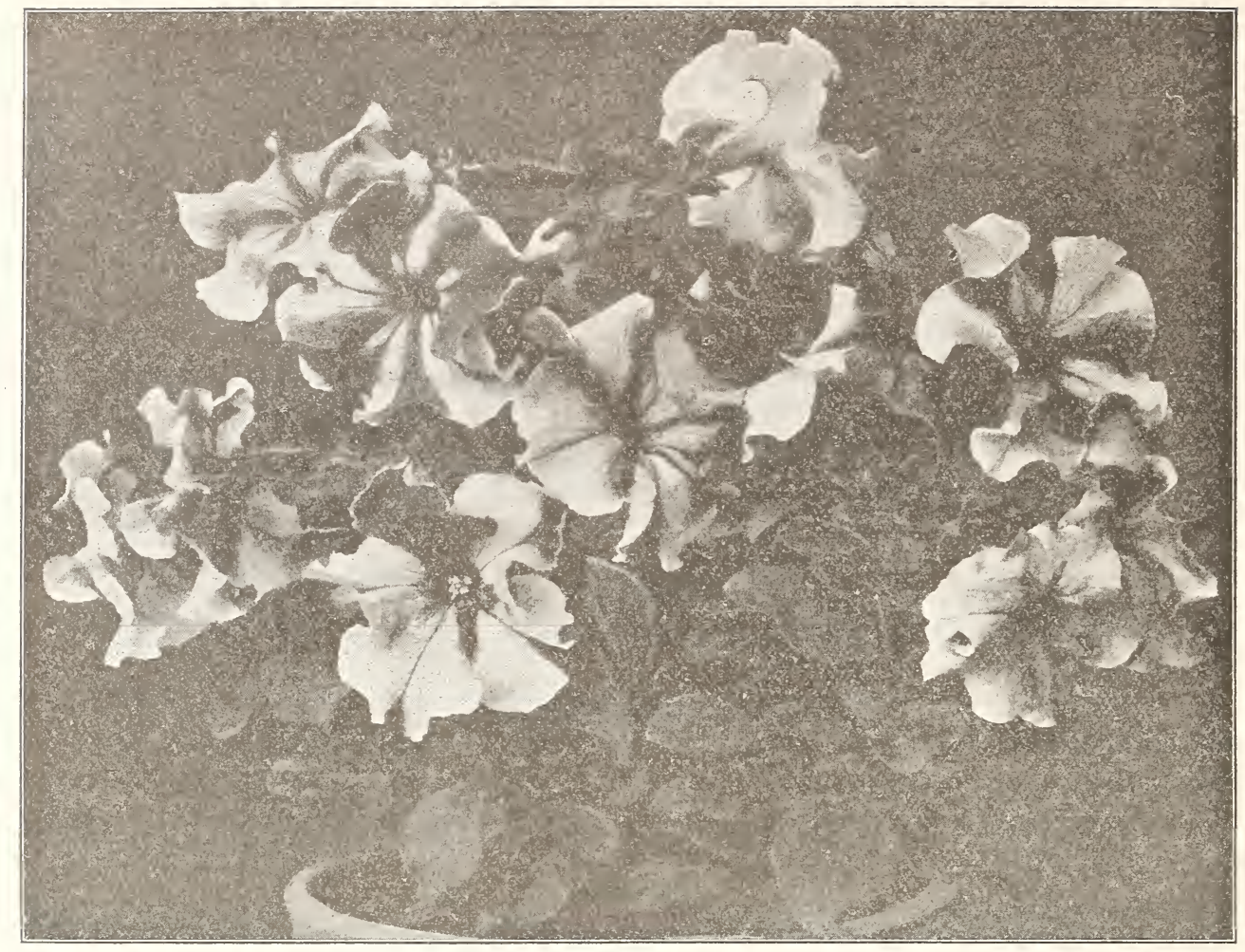

PETUNIA GRANDIFLORA NANA (Latest Novelty-Benary)

(Large flowering dwarf Petunias)

Since my dwarf Petunias have enjoye 1 a large demand for them for years I have deemed it advisable to introduce a low growing, large-flowered Petunia of excellent qualities. The plants will attain a height of only 8 to 9 inches, branch out considerable but will grow in close formation making it unnecessary to tie them. The pretty round bushes carry their flowers well above the foliage. Their colors are white and dark purple or mixed in beautiful combinations. They are suited especially for pot plant purposes or can be used in flower beds.

Trade packet, $\$ 2.00 \quad$ Five trade packets, $\$ 9.00$ 
Alba, pure white

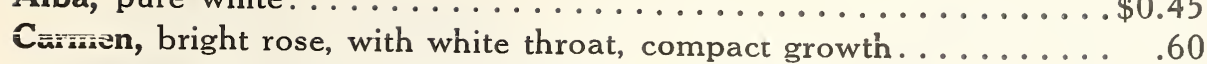

Carmen Sylvia, velvety violet, with pure white throat. . . . . . . . . 90

Flesh Colored, pink, pretty................... 1.30

Illustris, brilliant carmine, one of the most effective varieties

Maculata (Inimitable), beautifully striped and blotched crimson and

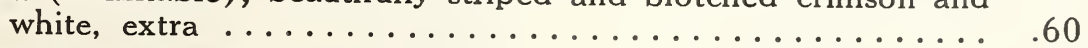

1.10

Maculata Purple-Star (Howards), deep purple, with white. . . . . . 1.00

Purple Violet, regular, compact and pyramidal habit.

Rosy Morn, flowers are brilliant rose, with large white throat and

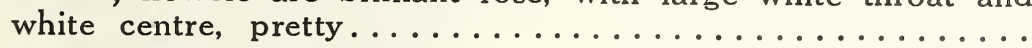

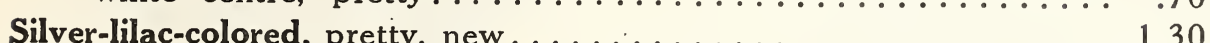

Undine, azure-blue

"Rose of Heaven. Dwarf, bushy plants. Flowers rich brilliant-

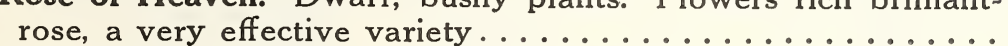

.. Snowball, very dwarf, white, rich flowering.............

“ Inimitable, dwarf, compact habit; profuse bloomer; flowers striped and blotched.

\section{PETUNIA hybrida pendula (Balcony Petunias)}

A splendid free flowering type for window or porch boxes, vases, etc., giving a display of flowers throughout the entire summer, until frost sets in; the flowers are large, averaging 3 inches across.

Alba, white $1 / 128$ oz.

\section{PETUNIA hybrida grandiflora \\ (Giant-flowered Single Varieties)}

Aurora, light rose, veined crimson-purple............. . \$1.25

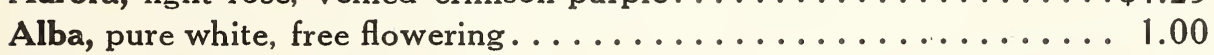

Brilliant-Rose, beautiful . . . . . . . . . . . . . . . . . 1.50

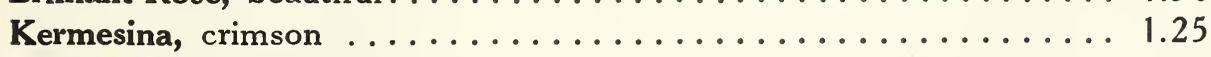

Kriemhilde, dark purple with large white throat. . . . . . . . 1.25

Maculata (Inimitable), striped and blotched crimson and white.....

Violacea albo-maculata (Admiration), deep velvety blue, white

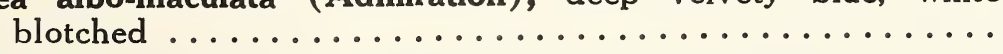




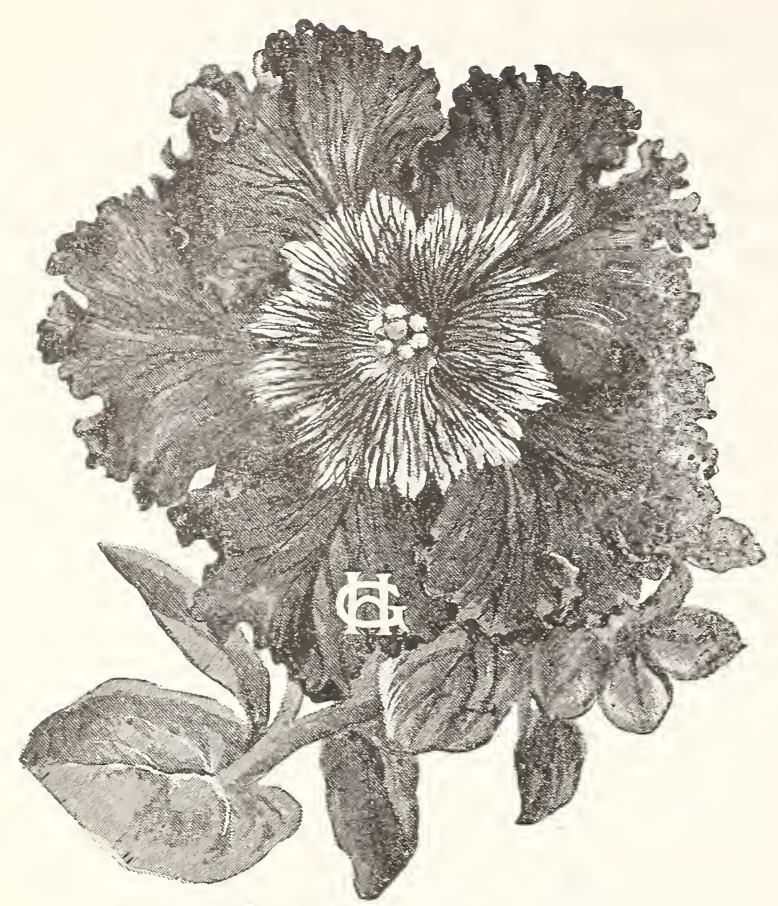

Petunia hybrida grandiflora fimbriata

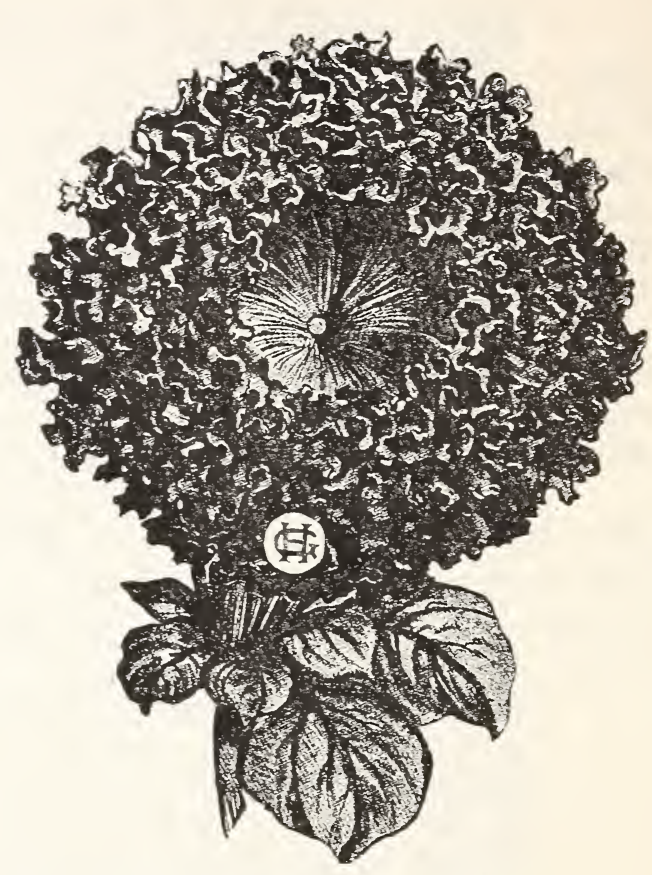

Petunia hybrida grandiflora superbissima

\section{PETUNIA hybrida grandiflora fimbriata (Ruffled varieties)}

Albo-lutea, whitish yellow, with yellow throat. . . . . . . $1 / 128$

Aurora, light rose, veined crimson-purple.............. 1.25

Brilliant-Rose, very beautiful. ................. 1.50

Coerulea, light blue with blackish-crimson veins.......... 1.25

Delicata, white and rosy-lilac, charming. ............. 1.50

Kermesina, fringed crimson, very fine.............. 1.50

Maculata, red and white blotched varieties, extra........... 1.25

Miranda, dark crimson, throat scarlet.............. 1.50

Pepita, flowers dark carmine, changing to scarlet, blotched white, charming .........................

Purpurea, purple-red, with dark throat. . . . . . . . . . . . 1.25

Rosalind, delicate rose. . ................... 1.50

Titania, royal purple, white-margined. . . . . . . . . . 1.25

Venosa, fine veined varieties...................... 1.25

Finest mixed (all colors).
$1 / 64$ oz.

$\$ 2.50$

2.50

3.00

2.50

3.00

3.00

2.50

3.00

3.00

2.50

3.00

2.50

2.50

2.50
1/32 oz. $\$ 5.00$ 5.00 6.00 5.00 6.00 6.00 5.00 6.00 6.00 5.00 6.00 5.00 5.00 5.00

\section{PETUNIA hybrida grandiflora superbissima}

(Ruffled)

Superbissima, deep rose, pretty ...................\$1.50

Alba, white with yellowish throat. .............. 1.50

$\$ 3.00$

$\$ 6.00$

3.00

6.00

“ Empress, whitish-blue, veined violet-blue, dark throat. . . . . . 1.50

3.00

6.00

“ Fimbriata, crimson, fringed................

“ Intus Aurea, splendid yellow throated varieties. . . . . . . . . . .

“ King Alphonso, dark purple, black throat, boldly waved and frilled ........................... 1.50

3.00

6.00

3.00

6.00

“ Coppery-Red, beautiful deep red color. . . . . . . . . . . . . 1.50

“ Princess of Wurttemberg, delicate rose, purple veined. . . . . . . 1.50

3.00

6.00

3.00

6.00

“ Princess of Wurttemberg, delicate rose, purple veined.......

" Quadricolor, bright rose, veined and netted carmine; throat blood-red with a star-like yellow centre............. 1.50

Silver-Rose, beautiful delicate color ................... 1.75 
PETUNIA hybrida grandiflora superbissima (continued)

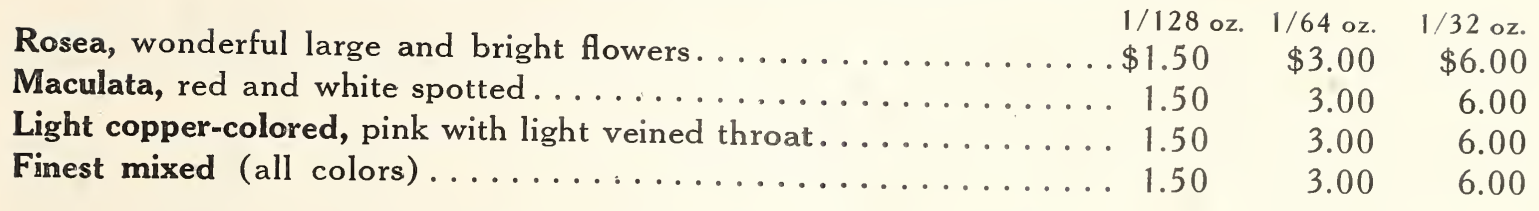

Petunia hybrida grandiflora superbissima nana

Splendid mixture of new compact growing superbissima Petunias. The height of this strain is only $1 / 2$ or $2 / 3$ of that of the old class. The flowers are very large but substantial, owing to their rich undulation which renders them loose and attractive. The display of colors is an extraordinarily rich one showing all shades, some with deep dark throats as well as with the most lovely veining.

$$
1 / 128 \text { oz., } \$ 2.00 \quad 1 / 64 \text { oz., } \$ 4.00 \quad 1 / 32 \text { oz., } \$ 8.00
$$

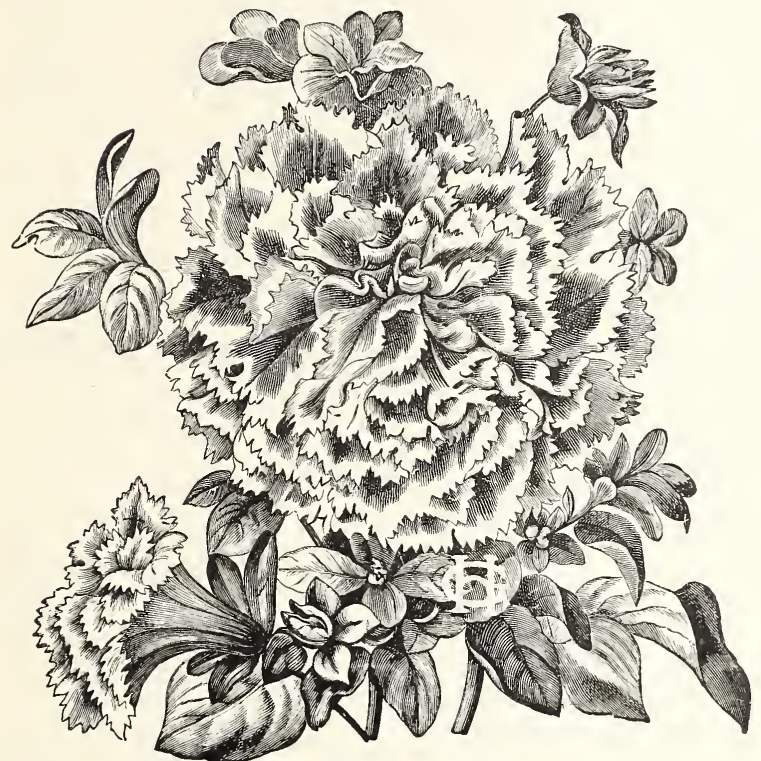

Petunia hybrida grandiflora double

\section{DOUBLE FLOWERING PETUNIAS}

As the seed of the double varieties is obtained by hybridization, it will not produce all double flowers, but the singles will be unusually fine. The weaker seedlings should receive great care, as they produce the finest double flowers.

Finest mixed

PETUNIA hybrida grandiflora f. pleno (double, large flowered)

Finest mixed 1000 seeds $\$ 2.00$

PETUNIA hybrida fi. pleno.

\section{(Double-flowered Varieties)}

Inimitable, fi. pleno, double, small flowering, purple and white blotched, charming .....................\$1.00 \$2.00 \$4.00

Liliput (nana comp. multiflora fl. pl.), Rose Bonheur, dwarf, compact

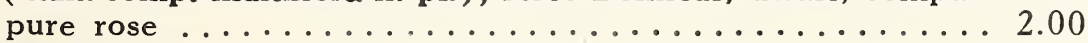

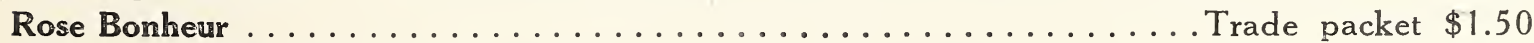
All sorts double small flowered mixed .................. Trade packet 1.50 
Berlin Specialty: Dark Red and Pink

(Special price on orders for $1 / 4$ ounce and more)

Mueller \& Mohnstein: Rosea and Atrosanguinea............ 1.25

Arends Specialty: Hamburg Red, brilliant dark red . . . . . . . . . 1.50

3.00

6.00

Rosea, rose .

2.50

5.00

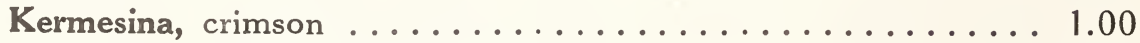

2.00

4.00

Fire Queen, glossy salmon-carmine.............. 1.50

3.00

6.00

Lilac

\section{LATEST NOVELTIES IN PRIMULA OBCONICA}

Hindenburg: Pink and dark red mixed............... \$2.00

Mackensen: Of dwarf compact growth. The plant blooms profusely, and the flowers are of very large size, carried above the foliage on very strong stems. The color of the blooms is a glossy deepcarmine-dark-blood-red .................... 2.00

Zeppelin: The plant is of very strong growth. Flowers are about 2 inches in diameter, carried on very strong stalks. The color of the blooms is a glossy salmon shading to purple red with salmon shine. (About 100 trade packages of this variety were distributed among florists of this country last year. It proved very satisfactory)

\section{PRIMULA MALACOIDES}

Malacoides. A charming pot plant, very early and free flowering, delicate lilac, new..................... \$0.50

Malacoides-Pink

Forbesi (Baby Primrose). Exceedingly free-flowering, flowers rosylilac; very useful for pots and bouquets, the seedlings begin to

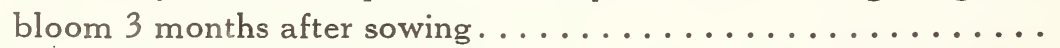

Forbesi Ruby, deep purple violet, golden yellow eyed, pretty . . . . . 1.00

Forbesi Rubra, red, very fine..................... . . . . . . . .

$1.50 \quad 3.00$

Kewensis, continuous-blooming, large-flowered, golden yellow, with magnificent foliage, very ornamental..............

\section{PRIMULA CHINENSIS FIMBRIATA}

\begin{tabular}{lllllll} 
Alba & Coccinea & Coerulea & \multicolumn{2}{l}{ Kermesina splendens } \\
Defiance & Morningred & Dornroschen & Brilliant Benary & $\$ 1.50$ & $\$ 3.00$ & $\$ 6.00$
\end{tabular}

\section{PRIMULA CHINENSIS FIMBRIATA FL. PLENO (Double)}

Alba (white) Rubra, Kermesina, Herzogin (white, with large bright carmine centre, foliage bronzy dark green). Finest mixed (all colors) 
Large Flowering Single, mixed . . . . . . . . . . . . . . $\$ 0.15$

Large Flowering Double, mixed. . . . . . . . . . . . . . . . 50

$1 / 2$ oz. $\quad 1$ oz.

$\$ 0.30 \$ 0.60$

i.

\section{PUERARIA}

Thumbergiana (Japanese Kudzu Vine), very useful climber, with Wisteria-like, purplish-violet, sweet-scented flowers......... \$0.25

$\$ 0.50 \$ 1.00$

\section{PYRETHRUM}

Parthenifolium Golden Feather, extra fine strain, pretty for borders. . \$0.15

$\$ 0.30 \$ 0.60$

\section{RHODANTHE (Everlasting)}

Maculata, $\operatorname{mixed} \ldots \ldots \ldots \ldots \ldots \ldots \ldots \ldots \ldots . \ldots \ldots \ldots$

\section{RICINUS (Castor Bean)}

Zanzibariensis, mixed. A very robust-growing and most elegant foliage plant. Leaves very large................. \$0.10

\section{SALPIGLOSSIS}

Variabilis grandiflora, mixed. Extra large-flowered........... \$0.25 superbissima, mixed. The largest flowered and most beautiful of all ........................... 40

\section{SALVIA}

Splendens compacta Dwarf Zurich, early and free flowering, brilliantred ........................... \$1.00 compacta erecta Bonfire, of quite upright growth, dark scarlet,

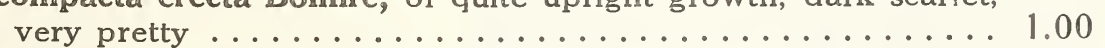
Glory of Stuttgart, large-flowered, brilliant scarlet, very beau-

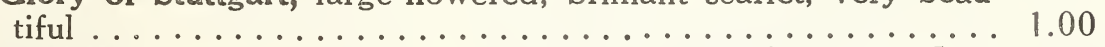

Fireball, of compact habit; very early and free-flowering; flowers of brilliant deep scarlet; of grand effect for bedding. . . . . .75

\section{SAPONARIA}

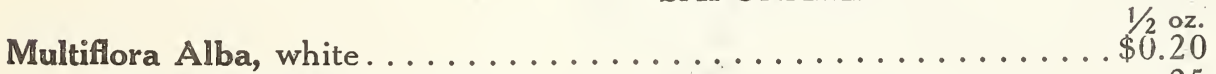

$1 \mathrm{oz}$.

" Scarlet Queen, very fine..................... . . . . . . . . . . .

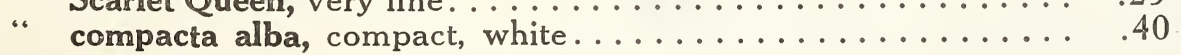

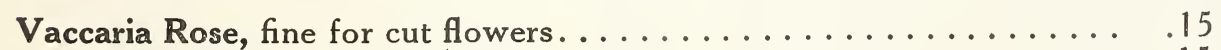

\section{SCABIOSA}

Tall Large-flowered Double Scabious

Black-purple and white, flesh color, cherry red, rose and crimson . . .\$0.25

$$
1 / 2 \text { oz. }
$$

1 oz. 


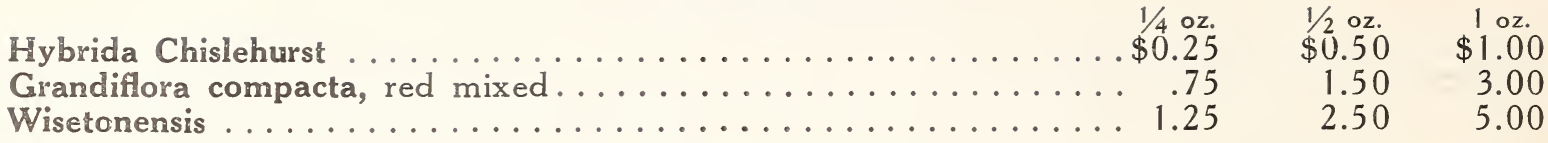

\section{SILENE}

Pendula (rosea graeca), bright rose double rose

White $\$ 0.10$

Ruberrima (carmine-rose) .... . 20

“ double rose

“ compacta rose

Bonnetti (dark purplish crimson). .20

Alba (white).............15

double white

Double white

.15

$\$ 0.20$
.40
.40
.30
.30

$\$ 0.40$

$40 \quad .80$
.40

$40 \quad .80$

$.30 \quad .60$

\section{SMILAX}

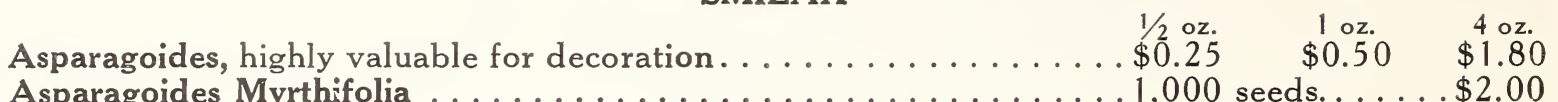

\section{STATICE}

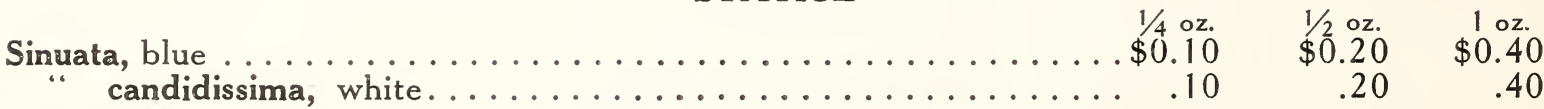

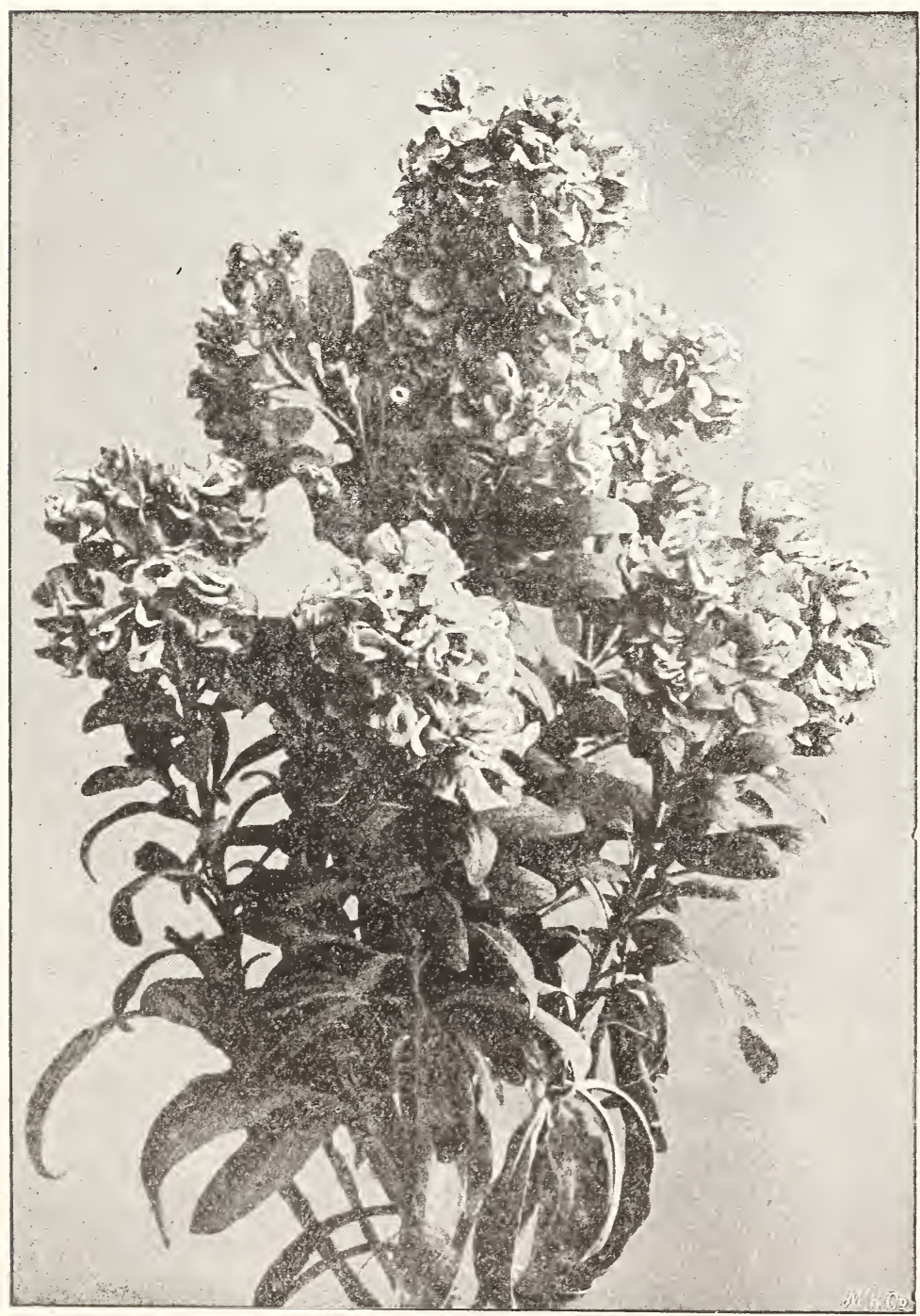

\section{STOCKS}

My collection of Stocks is made up of the finest and choicest varieties in existence. The seeds I offer are hand picked from plants carefully grown in pots, and will produce about $70 \%$ double flowers.

\section{Dwarf Erfurt 10 Weeks}

\begin{tabular}{|c|c|}
\hline $\begin{array}{l}\text { White } \\
\text { Salmon } \\
\text { Dark Blue } \\
\text { Purple } \\
\text { Pink } \\
\text { Lilac Rose }\end{array}$ & $\begin{array}{l}\text { Crimson } \\
\text { Canary Yellov } \\
\text { Blood Red } \\
\text { Light Blue } \\
\text { Carmine } \\
\text { Finest Mixed }\end{array}$ \\
\hline 1 & 0 \\
\hline
\end{tabular}

\section{Special Large-Flowering 10 Weeks}

$\begin{array}{ll}\text { White } & \text { Light Blue } \\ \text { Salmon } & \text { Canary Yellow } \\ \text { Purple } & \text { Blood Red } \\ \text { Lilac Rose } & \text { Crimson } \\ \text { Pink } & \text { Dark Blue } \\ \text { Carmine } & \text { Finest Mixed }\end{array}$

$1 / 4$ oz., \$1.25; 1/2oz., \$2.50;

1 oz., $\$ 5.00$ 
Dresden 10 Weeks

$$
\text { 1/4 oz. } \quad 1 / 2 \text { oz. } \quad 1 \text { oz. }
$$

This superb, large-flowered variety is of exquisite beauty, and

deserves the highest praise. It is almost a perpetual bloomer.

White Pink Purple Crimson

Carmine Salmon Blood Red Lilac Rose

Light Blue

Dark Blue Canary Yellow

Mixed ....\$1.00

$\$ 2.00 \$ 4.00$

\section{Excelsior}

This magnificent variety produces only a single, long and sturdy spike, closely set with enormous sized well-shaped double

flowers.

White Pink Purple Crimson

Carmine Salmon Blood Red Lilac Rose

Light Blue Dark Blue Canary Yellow

Mixed ...\$1.50 \$3.00

$\$ 6.00$

Bismarck 10 Weeks

An exceptionally fine new Ten-Week Stock, of very robust growth and pyramidal habit. It is perpetual flowering, blooming from June till September or October without interruption.

White

Pink

Purple

Crimson

Carmine

Salmon

Blood Red

Lilac Rose

Light Blue

Dark Blue

Canary Yellow

Mixed ....\$1.25

$\$ 2.50$

$\$ 5.00$

Brompton, forcing

White

Carmine

Light Blue
Pink

Salmon

Dark Blue
Seeds

1,000

Purple

Blood Red

Canary Yellow
Crimson

Llilac Rose

Mixed ....\$0.75
Seeds

5,000

\section{Dwarf Winter, forcing}

$\begin{array}{llll}\text { White } & \text { Pink } & \text { Purple } & \text { Crimson } \\ \text { Carmine } & \text { Salmon } & \text { Blood Red } & \text { Lilac Rose } \\ \text { Light Blue } & \text { Dark Blue } & \text { Canary Yellow } & \text { Mixed ..... }\end{array}$

$\begin{array}{ll}\text { White } & \text { Pink } \\ \text { Carmine } & \text { Salmon } \\ \text { Light Blue } & \text { Dark Blue }\end{array}$

Purple

Canary Yellow

Lilac Rose

\section{Nice Early Blooming}

White

Pink

Carmine

Salmon

Purple

Blood Red

Crimson

Light Blue

Dark Blue
Lilac Rose

Mixed .... . .60

SWAINSONIA

Superba

\section{TAGETES (See Marigold}

THUNBERGIA

Alata, yellow .......................\$0.30

$\$ 0.60$

.60

$\$ 1.20$

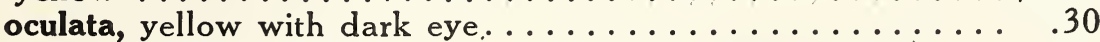

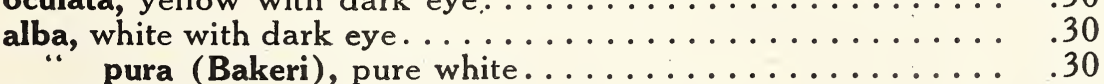

$\cdots$

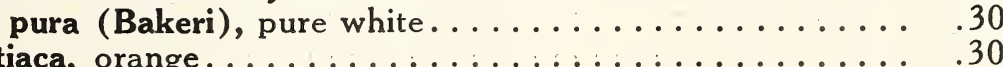

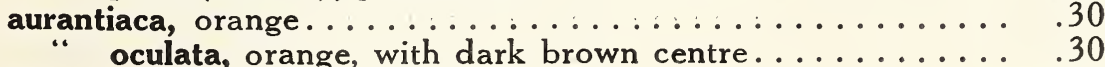

“ Fryeri, golden yellow, with white eye.............. 30

“. Finest mixed 
Asta Ohn. Lavender

Aurora. Striped or flaked orange-scarlet on a white ground . . . . . . . . .60

Blue Bird. A magnificent violet-blue . . . . . . . . . . . . . . . . . . . . . . . 60

Blue Wonder. An extra large free blue.

Blue Jacket. Rich, dark navy blue.

Burpee's Orange. Glistening orange of outstanding purity.

Columbia. The best and most productive pink and white.

Eldorado. Pure orange-salmon. An improved Mrs. Kerr

Fair Maid. Blush or light pink.

Fire King. Orange-scarlet.

Flamingo. An extra large orange-suffused salmon rose $\ldots \ldots \ldots \ldots \ldots \ldots \ldots \ldots .6 . \ldots \ldots$

Glitters. Bright, fiery, orange-shaded scarlet .

2.00

1.75

2.00

Gorgeous. Large, rich salmon cerise.

2.00

Glorious. Clear, rosy purple.

.60

.60

Grenadier. A glowing poppy scarlet, with extra long graceful stems.

Harlequin. Striped or flaked deep chocolate on a light ground . . . . . . . . 60

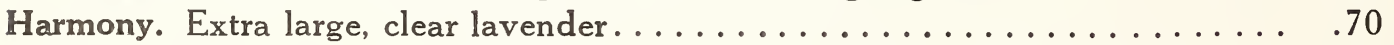

Irene. An early lavender Geo. Herbert. . . . . . . . . . . . . . . . . . . . 50

Lavender King. The most profitable lavender . . . . . . . . . . . . . . .60

Liberty. A very good early red . . . . . . . . . . . . . . . . . . . 50

Madonna. Best black-seeded white to date. . . . . . . . . . . . . . . 50

Miss Louise Gude. Brilliant pink.

Miss Spokane. Peach red. Wings bright scarlet, blending into an effect

Mrs. Harding. The famous Harding or Silver Blue . . . . . . . . . . . . . . .60

Mrs. Kerr. A clear, rich salmon. ..................... .80

Mrs. Chas. A. Zvolanek. Clear lavender.

Mrs. Skaach. Flowers bright shell-pink.

Mrs. M. Spanolin. Black-seeded white. . . . . . . . . . . . . . . . 50

Princess. Rich dark lavender, very free flowering, of medium size.

Rose Queen. The finest light rose pink.

Snowstorm. The standard pure white.

True Blue. A very fine, clear, even blue of fair size, an extra free

\section{bloomer}

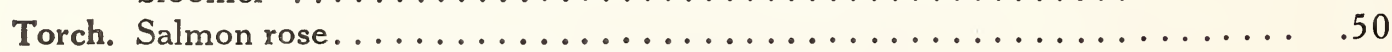

Vulcan. A large, brilliant, scarlet orange. . . . . . . . . . . . . . . .

White Star. Black-seeded white.

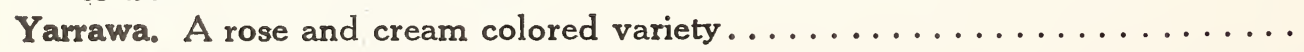

Zvolanek Rose. Extra large deep rose. . . . . . . . . . . . . . . . . . . . .60

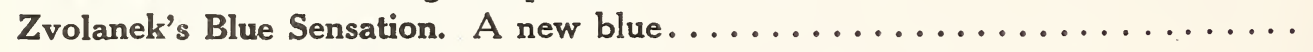

Zvolanek's Blue. Bright blue. . . . . . . . . . . . . . . . . . . . 
Bailloni, yellow with brown

Fournieri, velvety blue, very charming.

speciosa (Giant Torenia), a most charming variety with enormous-sized flowers. . . . . . . . . . . . . . . . . 1.00

\section{TROPAEOLUM}

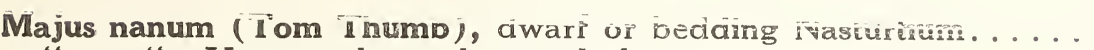

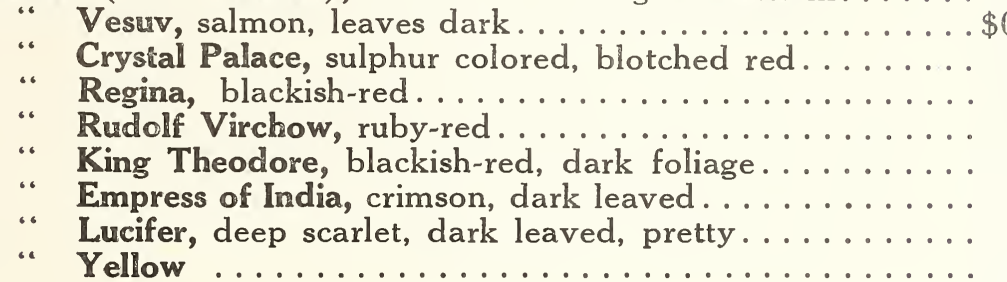

\section{VERBENA}

Hybrida auriculaeflora, fine Auricula eyed . . . . . . . . . . \$ $\$ 0.50$

candidissima, pure white, large flowered . . . . . . . . . . . 50

coccinea, scarlet, large flowered................ .50

oculata (Auric, coccinea), white eyed.......... .50

coerulea, blue, large flowered................. 50

“. oculata, dark blue, white eyed.............. 50

“ lutea, pale yellow . . . . . . . . . . . . . . . . . . . . . . . . . 50

“ Purple-cloak, dark purple, very fine............... 50

“ rosea, fine rose colored..................... 50

“ black blue with white eye, very beautiful............ 50

“ striata, striped varieties................... 50

“ splendid mixed...................... 50

Hybrida grandiflora auriculaeflora, auricula eyed ........... . . 50

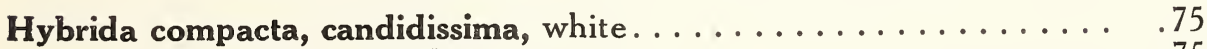

Venosa, violet, veined, pretty.................. 50 
Rosea (Periwinkle), rose...................... $\${ }^{1 / 4} 0.25$

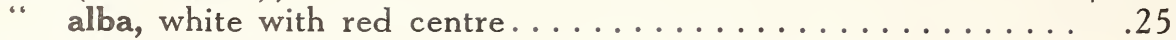

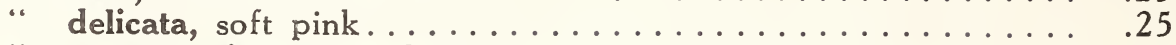

“ nova species, pure white.................... . 25

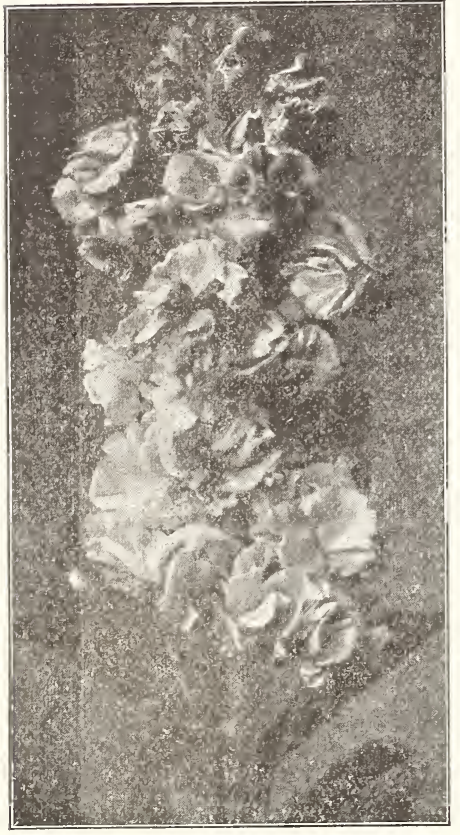

Double Wallflower

Fruehwunder

\section{WALLFLOWER (Goldlack)}

\section{Double Fruehwunder (Latest Novelty)}

Planted in January or February, these plants will bloom in June, planted in March they will bloom in July, etc., Thus, it will be seen that it is possible to have blooming plants during the entire year. It is to best advantage to sow in June, July, August and September in order to have blooming plants outside in the fall or in the cool houses in winter or spring. Fruehwunder will grow to a height of 30 inches. It will branch out, these branches also being laden with beautiful flowers which are well suited for cut flower purposes during the cool season.

This new variety comes in Canary Yellow, Gold Yellow, Bronze, Light Brown and Dark Brown. Seeds may be had in a mixture of the above or in Gold Yellow or Brown only.

Trade packet, $\$ 1.50$. Five trade packets, $\$ 7.00$.

\section{Single Varieties}

$$
1 / 4 \text { oz. }
$$

Berlin, glowing brown, large flowered............\$0.75

Ruppert, early, dark brown, very large flowered.......... .75

Harbinger, early, brilliant-brown............... .75

Fire King, carmine on orange ground . . . . . . . . . . . 75

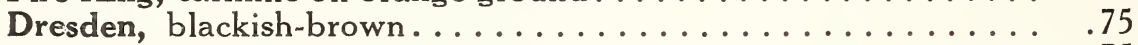

Ruby Gem, ruby violet with blue . . . . . . . . . . . . . 75

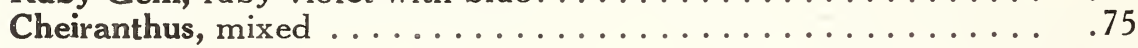

Double Varieties

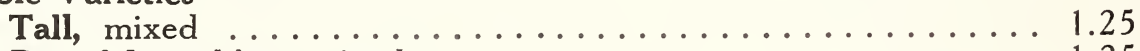

Dwarf branching, mixed.

\section{ZINNIA elegans double}

\section{Giant Zinnia-Dahlia Flowered}

$\begin{array}{lll}\text { White } & \text { Rose } & \text { Crimson } \\ \text { Golden Yellow } & \text { Scarlet } & \text { Isabella (delicate light yellow) }\end{array}$

Golden Yellow Scarlet lsabella (delicate light yellow)

Finest mixed $\ldots \ldots \ldots \ldots \ldots$. . . . . . . . . .

Giant Zinnia (grandifloria robusta
White

Violet

Carmine

Flesh color

Rose
Sulphur Yellow

Crimson

Gold Yellow
$\$ 0.75$

$\$ 1.50$

$\$ 3.00$

$\$ 1.50$

1.50

1.50

1.50

1.50

1.50

1.50

2.50

2.50
$1 \mathrm{oz}$.

$\$ 3.00$

3.00

3.00

3.00

3.00

3.00

3.00

5.00

5.00

Liliput (extra fine, small, very regular, double flowers)
White
Orange
Scarlet
Lilac

Golden Yellow

Splendid double mixed.

Finest mixed.... $\quad .50$

\section{XERANTHEMUM}

Double white, double purple, double rose. . . . . . . . . . . . . . 25 
Adiantum chilense

Trade packet $\$ 0.75$

Capillus veneris

Trade packet

1.00

imbricatum

Trade packet

1.00

cuneatum

Trade packet

elegans

gracillimum

Trade packet

formosum

Trade packet

fragrantissimum

Trade packet

Griesbaueri

Trade packet

Mackayasum

Trade packet

Matador

Trade packet

1.50

Mundulum

Trade packet

1.50

rhodophyllum

Trade packet

rubellum

Trade packet

Scutum

Trade packet

Triumph (Concordia)

Trade packet

1.00

Weigandii

Trade packet

2.00

Williamsii

Trade packet

Trade packet

Alsophila australis (greenhouse tree fern)

Trade packet

Aspidium Filix mas (outdoor)

Trade packet

spinulosum (outdoor)

Trade packet

“ Thelypteris (outdoor)

Trade packet

violascens (greenhouse)

Trade packet

Asplenium bulbiferum (greenhouse)

Trade packet

Filix femina (outdo.or)

Trade packet

Hillii (greenhouse)

Trade packet

Hookeri (greenhouse)

Trade packet

laxum (greenhouse)

Trade packet

" Nidus Avis (greenhouse)

Trade packet

Ruta muraria (outdoor)

Trade packet

Blechnum brasiliense (greenhouse tree fern)

Trade packet

" longifolium (greenhouse)

Trade packet

Cyrtomium Fortumei (greenhouse)

Trade packet

Rochfors:i (greenhouse)

Trade packet

falcatum (outdoor)

Trade packet

Lastraea aristała variegata (greenhouse)

Trade packet

Lomaria ciliata (greenhouse tree fern)

Trade packet

Nephrolepis Piersonii (greenhouse) .................. Trade packet

Pellaea hastata (greenhouse)

Trade packet

Platypodium aureum (greenhouse)

Trade packet

Reinwardii (greenhouse)

. Trade packet

repandum (greenhouse)

. Trade packet

Platycerim alcicorne (greenhouse)

Trade packet

Pteris aquilina (outdoor)

. Trade packet

cretica major (Quvrardia) (greenhouse) ................ Trade packet

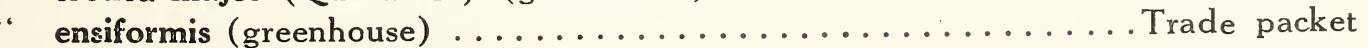

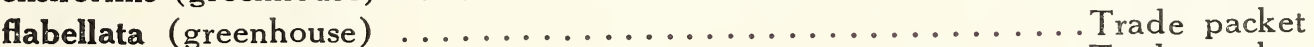

“ internata (greenhouse) $\ldots \ldots \ldots \ldots \ldots \ldots \ldots$. . . . . . . . . . . . . . . . . . . . . . . . . . . . . . . . . . . . . .

“ Mariesii (greenhouse) ........................ Trade packet

Struthiopteris germanica (outdoor) .................. Trade packet

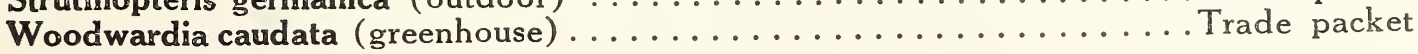




\section{ORNAMENTAL GRASSES (Annuals)}

Agrostis dulcis (Sweet Bent Grass) . . . . . . oz. 1 oz.

Agrostis dulcis (Sweet Bent Grass) . . . . . . . . . . . . . . . . \$0.25

laxiflora (Lax (Loose)-flowered Bent Grass), pretty . . . . . . . . . . . .25

nebulosa (plumosa) (Cloud Grass), very graceful . . . . . . . . . . . . . .50

pulchella (Pretty Bent Grass), very fine. . . . . . . . . . . . . . . 1.00

1.00

verticillata (Bent Grass) . . . . . . . . . . . . . . . . . . . . 30

2.00

.60

Anthoxanthum gracile (Border Grass), fine . . . . . . . . . . . . . . . . . . .75

Avena sterilis (Animated Oat), for large bouquets . . . . . . . . . . . . . 15

1.50

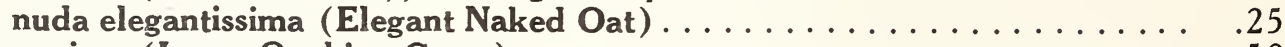

.30

.50

Briza maxima (Large Quaking Grass).

.50

minima (Small Quaking Grass) . . . . . . . . . . . . . . . . . . 1.00

1.00

Bromus brizaeformis

macrostachyus (lanuginosus), beautiful f. bouquets . . . . . . . . . . . . . . 25

madritensis (Madrid Brome), beautiful f. bouquets. . . . . . . . . . . . . .

patulus (Spreading Brome), beautiful f. bouquets .

nanus (dwarf), beautiful f. bouquets................ 50

purpureus, beautiful $\mathrm{f}$. bouquets. . . . . . . . . . . . . . . . . . . . . . .

2.00

1.50

Diplachne fascicularis, for bouquets.....................

Eleusine barcinonensis, beautiful grass.

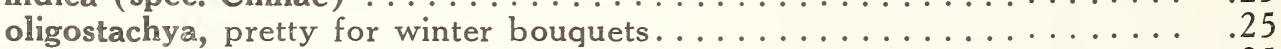

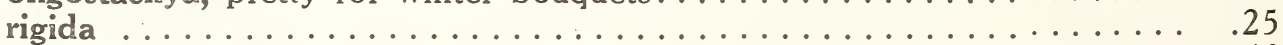

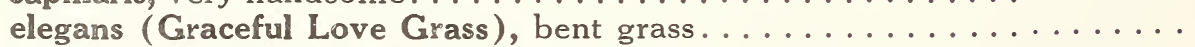
maxima (Large Love Grass), pretty.

Festuca procumbens, very dwarf.

Stipoides, pretty for bouquets.

Hordeum jubatum (Squirreltail), nice for bouquets.

Panicum capillare violaceum (Violet Hair-Stalked Panick Grass)

Paspalum elegans (Elegant Millet Grass), for bouquets.

Poa pilosa, pretty for bouquets and borders.

Setaria macrochaeta, nice for bouquets. . . . . . . . . . . . . . . . 25

alopecuroides (Fox-Tail-like Bristle Grass) . . . . . . . . . . . . . . . . . . . . . . . .

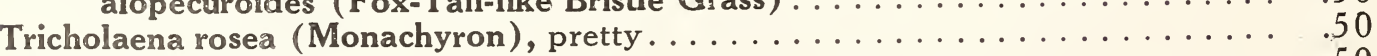

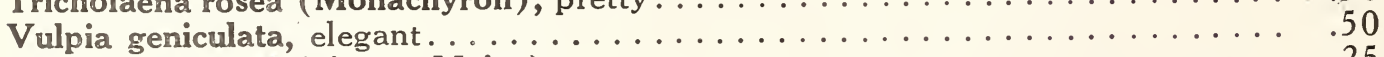

Zea gracillima (Miniature Maize) . . . . . . . . . . . . . . . .

$$
\text { variegata, very nice. . }
$$




\section{PERENNIAL FLOWER SEEDS}

\section{ACHILLEA}

Ageratum, flowers yellow, sweet scented..$r$ oz.

Ptarmica double (Sneezewort), white, a fine cut flower . . . . . . . . . .70

“ " "The Pearl," pearl-white............... 1.00

Vulgaris plenissima ....................... 50

Filipendula, golden yellow . . . . . . . . . . . . . . . . 50

\section{ACONITUM (Monkshood)}

Lycoctonum (True Wolf's-Bane), yellowish flowers..........\$0.75

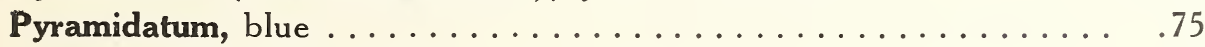

\section{ANCHUSA}

Barrelieri, dwarf, early flowering, blue. . . . . . . . . . \$ 0.40

\section{ANEMONE (Wind Flower)}

Sinensis (coronaria) von Caen, fine large flowering mixed....... \$0.30

\section{AQUILEGIA (Columbine)}

Hybrida double, beautiful colors. . . . . . . . . . . . . \$ 0.50

Chrysanta, bright yellow . . . . . . . . . . . . . . . . 50

\section{ALYSSUM}

Argenteum, leaves white, flowers yellow ............. $\$ 0.30$

\section{ARABIS (Rock Cress)}

Alpina, very early flowering, flowers white, foliage whitish

\section{ASPHODELUS}

Luteus, shining yellow 
Alpinus Goliath, bright blue, largest flowers............... $1 / 4 \mathrm{oz}$.

" Nixe, flowers star-shaped, light blue ............. .50

“ speciosus, large flowering, beautiful .............. 50

1.00

2.00

" superbus, light blue...................... . . 50

Amellus roseus, light rose, pretty . . . . . . . . . . . . . . 1.60

3.10

6.00

Bessarabicus, dwarf, deep purple, beautiful. . . . . . . . . . . 1.60

3.10

6.00

Cassubicus, lavender, large flowering . . . . . . . . . . . . . . 1.60

3.10

Hybridus semiplenus, semi-double hybrids, new .

2.25

4.25

6.00

8.00

\section{ASTILBE}

Davidi, graceful spikes of rosy-violet, or mauve flowers

\section{AUBRIETIA}

Graeca, light blue, pretty miniature plant........... $\$ 1 / 4$ oz.

\section{BIDENS}

Atresanguinea (Dahlia Zimapani) dark blood red . . . . . . . . . . \$0.90 \$1.80

BOCCONIA (Plume Poppy)

Japonica (cordata), a handsome border plant, cream colored.....\$0.30

\section{CAMPANULA (Bell Flower)}

Medirm.single carmine rose lilac blue...........\$0.75

blue............ 1.00

\section{Calycanthema (Cup and Saucer), carmine}

rose

lilac blue

Pyramidalis, blue

\section{CANDYTUFT (Iberis)}

Gibraltarica, lilac, changing to white............... \$0.50

“ hybrida, dwarf, free flowering................ . . 40

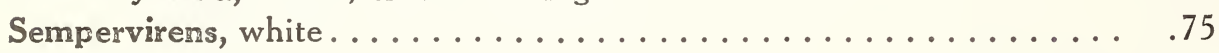

\section{CENTAUREA}

Calocephala

\section{CERASTICUM}

Tomentosum, snow white.................... $\$ 0.40$

Biebersteini, flowers and foliage white.

\section{CHAMAEPEUCE}

Diacantha, purplish flowers, silvery-striped foliage

\section{CHEIRANTHUS}

Allionii, bright orange yellow, fine rock or border plant 
Barbata (Pentstemon)

\section{@OREOPSIS (Tickseed)}

Grandiflora, large flowered, bright yellow, pretty cut flower.

\section{DELPHINIUMI (Perennial Larkspur)}

Hybrida Belladonna, light azure-blue, large flowered......... \$ 0.75

Formosum, dark blue, pretty plants............... . 75

“ Sulphureum, large, pure sulphur-yellow flowers on long spikes,

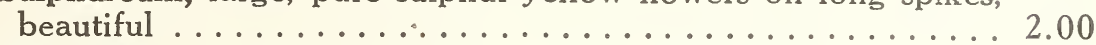

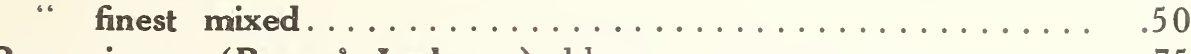

1.00

Brunonianum (Brown's Larkspur), blue.............. .75

Chinense, pretty colors..................... .50

Nudicaule, beautiful scarlet. . . . . . . . . . . . . . . 1.50

Speciosum, dark blue

\section{DIANTHUS (Sweet William)}

Barbatus Dunnett's, double dark crimson, very effective........ . \$0.20

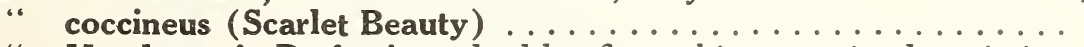

Henderson's Perfection, double, fine white margined varieties,

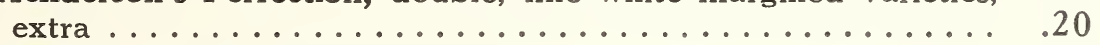

nigricans, double, black red . . . . . . . . . . . . . . . . 20

roseus splendens (Pink Beauty), fine pink shade. . . . . . . . . 20

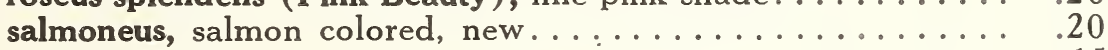

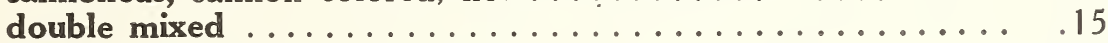

single mixed

\section{DIGITALIS (Foxglove)}

Purpurea gloxiniaeflora, purple................. $\$ 0.15$

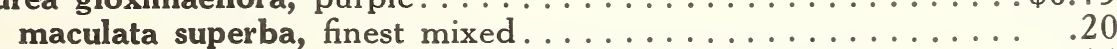

“ monstrosa, finest monstrous-flowered varieties mixed....... . 15

\section{DRACOCEPHALUM}

Ruyschianum, dark blue. . . . . . . . . . . . . . . . \$ 0.25

Virginianum (Physostegia), rosy-lilac............... .50

album, white, fine cut flower................ 50

\section{ECHINACEA (Coneflower)}

Purpurea, a very handsome border plant bearing large heads of rosypurple flowers and dark central disc...........\$0.25

\section{ERIGERON (FLEA BANE)}

Coulteri, very early, white, fine for cutting. . . . . . . . . . \$0.75

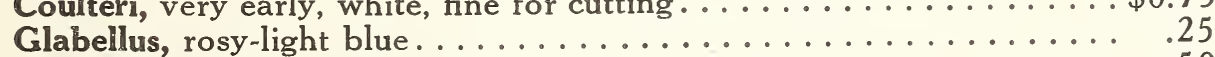

Grandiflorus elatior, early flowering, lilac. . . . . . . . . . . . . 50

Multiradiatus roseus, bright rose, graceful cut flower. . . . . . . . . 1.00

Speciosus hybridus grandiflorus, lilac and blue shades.......... . . . 40

Speciosum (Stenactis Speciosa).

\section{ERYSIMUM}

Pulchellum, very free flowering, yellow. 


\section{ERYNGIUMi (Sea Holly)}

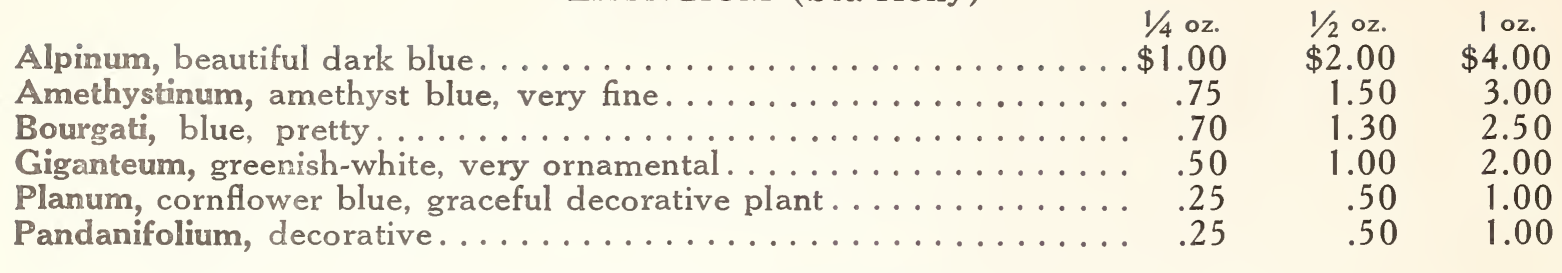

\section{ECHINOPS (Globe Thistle)}

Exaltatus, light blue........................ . . . . . 15

Giganteus, light azure blue....................... . . . . . . . . . .

$\$ 0.25$

$\$ 0.50$

.25

.35

Ritro, metallic blue flower heads................... . 20

Humilis Cyane, dwarf, light blue, very ornamental. . . . . . . . . . .20

\section{FUNKIA (Day Lily)}

Coerulea, blue ......................... $\$ 0.20$

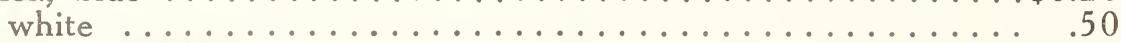

\section{GAILLARDIA (Blanket Flower)}

Grandiflora, sanguinea, large flowers, blood red, yellow border... . \$0.20

oculata, very fine new hybrids, light shades with red centre... .20

“ Standhalter, giant flowering, long stems............. . . 25

Bicolor, yellow and brown.

\section{GERBERA}

Jamesoni (Barberton Daisy). A beautiful plant, with long-stalked,

\section{GEUM}

Coccineum atrosanguineum double, $\underset{\text { " }}{\text { orange-red } \ldots \ldots \ldots \ldots} \ldots \ldots \ldots \ldots$

(Mrs. Bradshaw), large flowered,

\section{GLADIOLUS}

Hybridus grandiflorus praecox, very beautiful

\section{GNAPHALIUM}

Decurrens, white, everlasting................. $\$ 1 / 8$ oz.

Foetidum, yellow everlasting.

\section{GYPSOPHILA}

Paniculata, whitish, beautiful for bouquets (Baby's Breath) ...... $\$ 0.10$

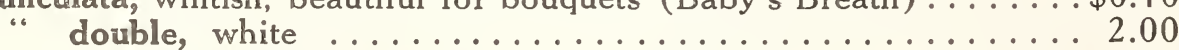

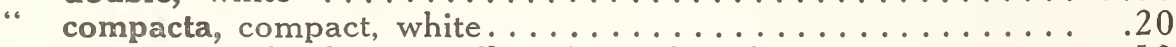

Repens, very dwarf, white, excellent for rockwork.......... .50

Acutifolia, lilac-rose .......................... . 40

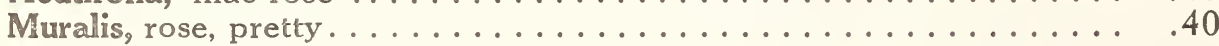

$1 / 2$ oz. 
Bigelowii, yellow, beautiful f. cutting. ............... 1/4 oz. $1 / 2$ oz. 1 oz.

HELIANTHUS (Hardy Sunflower)

Annuus gigantus, flowers of enormous size. . . . . . . . . \$0.25 \$0.50 \$1.00

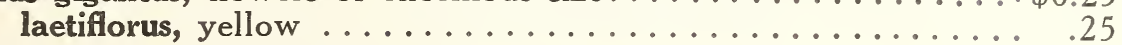

$.50 \quad 1.00$

HELIANTHEMUM (Sun Rose)

Mutabile, various, beautiful.

$\$ 0.50$

$\$ 1.00$

\section{HELIOPSIS}

Scabra, yellow, an excellent cutflower...............\$0.25 \$0.50 \$1.00

\section{HELLEBORUS}

Niger (Christmas Rose), white.................\$0.25

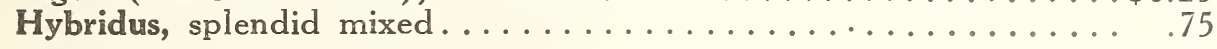

$\$ 0.50$

1.50

$\$ 1.00$

3.00

HEMEROCALLIS (Yellow Day Lily)

Flava, (Orange-yellow-Day Lily) ................. \$0.75

Middendorfii, golden yellow................... . 50

\section{HESPERIS MATRONALIS}

\section{(Sweet Rocket-Evening Scented Stock)}

Purple ............................ \$0.15

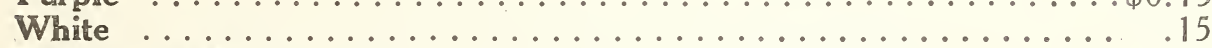

Nana Candidissima, dwarf, pure white, early flowering.......... .30

Tristis (Sweet Rocket), brown................... 10

\section{HEUCHERA (Alum Root)}

Rosea, rose, charming.......................\$1.00

Sanguinea, deep red, very effective............... 1.50

\section{HIBISCUS (Rose Mallow)}

Albus grandiflorus, large flowered, white, splendid ..... $\$ 0.20$

Roseus grandifforus, flowers large, glossy rose.............. . . . . . . . . . .

Moscheutos (Rose Mallow), pink flowers................... . . 40

Manihot, golden yellow, large flowers.................. . . 30

\section{HOLLYHOOK}

Double mixed

Single mixed

\section{HYACINTHUS}

Candicans (Cape Hyacinth), white, very decorative........\$0.25

\section{HYPERICUM (St. John's Wort)}

Elegans, an elegant hardy perennial with yellow flowers $\$ 0.40$ 
Kaempferi (Japanese Iris), finest varieties mixed . . . . . . . . $\$ 0.40$

\section{IBERIS (See Candytuft)}

\section{JULIENNE}

Maritimus (Virginian Stock) .

\section{LATHYRUS (Hardy Pea)}

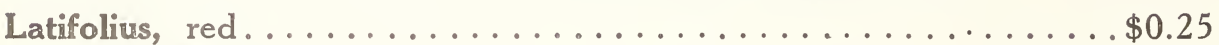

" roseus, bright rose....................... . . . . . .

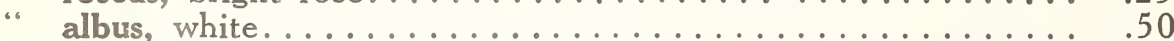

“ White Pearl, very large flowering. . . . . . . . . . . . . . .65

Rotundifolius, deep rose colored.................. .20

$\$ 0.50 \$ 1.00$

$.50 \quad 1.00$

$1.00 \quad 2.00$

$1.30 \quad 2.50$

$.40 \quad .80$

\section{LEUCANTHEMUM (Shasta Daisy)}

Maximum hybridum King Edward, large flowered, pretty . . . . . . . \$0.50

" "Westralia, white, semi-dwarf........... 50

“ " Alaska, beautiful.................. 50

" "California, yellow, changing to white........ 50

“ Mayfield Giants, snow-white.................. 50

“ Mrs. C. Lowthian Bell, beautiful. . . . . . . . . . . . . . . 50

$\$ 1.00 \$ 2.00$

$1.00 \quad 2.00$

$1.00 \quad 2.00$

$1.00 \quad 2.00$

$1.00 \quad 2.00$

$1.00 \quad 2.00$

LIATRIS (Gay Feather)

Scariosa praecox, purple red, fine.

$\$ 0.25$

$\$ 0.50$

$\$ 1.00$

LINUM (Flax)

Perenne, blue, a graceful hardy perennial.

\section{LUPINUS}

Arboreus (Tree Lupin), yellow.................. . \$0.20

“ albus (Snow Queen), pure white, magnificent......... 40

$\$ 0.40$

$\$ 0.75$

.80

1.50

1.50

3.00

Grandifolius, purple......................... . . 20

.40

.75

Polyphyllus Douglasii superbus, violet blue and white......... . . 20

Goldspitze, new, glowing golden yellow w. blue........ . 75

.40

.75

1.50

3.00

" Lavender with white and rose shade............... .75

“ Moerheimi, beautiful soft pink................... . . 75

" mixed

.70

1.50

3.00

1.50

3.00

1.40

2.75

\section{LYCHNIS}

Viscaria splendens, brilliant red flowers...............\$0.25

$\$ 0.50$

$\$ 1.00$

\section{MYOSOTIS (Forget-Me-Not)}

Alpestris, Blue

. Alpestris, Rose.

$\$ 0.40$

$\$ 0.80$

$\$ 1.50$

\section{PAEONIA}

Albiflora, early flowering, finest mixed-single. 
Orientale (Oriental or Eastern Poppy), dark red.......... \$0.50

Nudicaule aurantiacum, orange-scarlet................. . 10

croceum (Iceland Poppy), yellow................ 10

\section{PENTSTEMON (Beard Tongue)}

Gentianoides, splendid mixed.................. \$0.40

“ grandiflorus, giant flowered mixed ............. .50

Pulchellus hybridus.

PHLOX

Decussata, newest and choicest mixed.............\$1.50

Dwarf, newest and choicest mixed.

2.00

PHYSALIS

Francheti (Chinese Lantern Plant), fruits very large......... . \$0.25

Bruniati (Miniature Lantern) . ................. 50

$\$ 0.50$

1.00

$\$ 1.00$

2.00

\section{PHYTOLACCA}

Kaempferi (acinosa), crimson berries

$\$ 0.10$

$\$ 0.20$

$\$ 0.40$

PHYSOSTEGIA (False Dragon Head)

Virginianum, $\operatorname{rosy}-$ lilac....................\$0.50

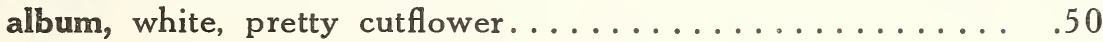

$\$ 1.00$

1.00

$\$ 2.00$

2.00

PLATYCODON (Chinese Moon Flower)

Grandiflora, large flowering, blue................. . . 0.75

$\$ 1.50$

$\$ 3.00$

POTENTILLA (Cinquefoil)

Calabra, yellow......................... $\$$. . 0.50

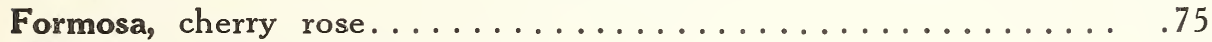

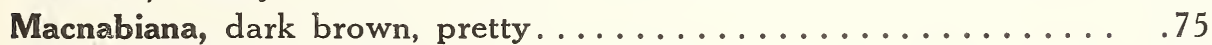

Pyrenaica, canary yellow.................... . 50

$\$ 1.00$

$\$ 2.00$

$1.50 \quad 3.00$

$\begin{array}{ll}1.50 & 3.00\end{array}$

$1.00 \quad 2.00$

PRIMULA (Hardy Primrose)

Auricula, splendid mixed. ................... $\$ 2.50$

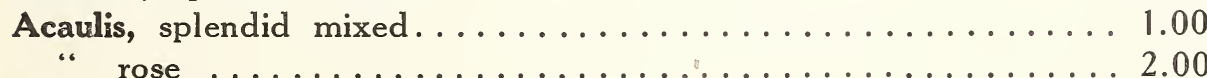

\section{PYRETHRUM}

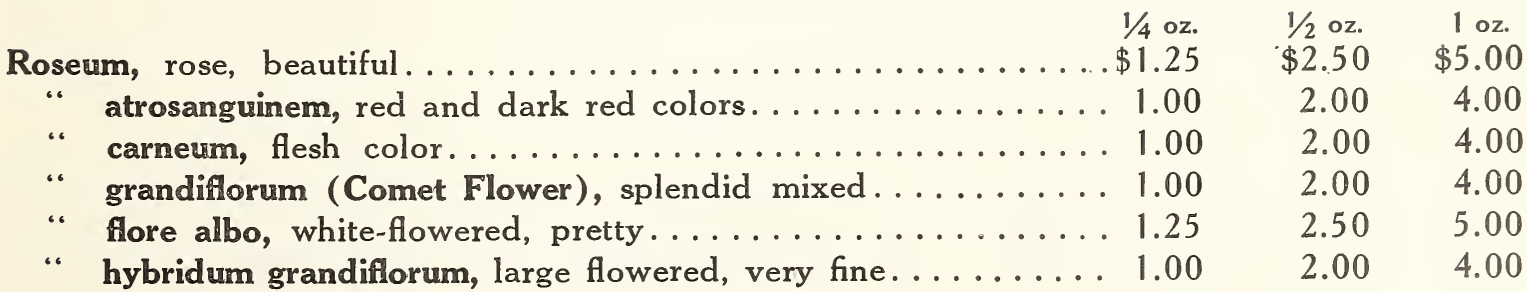




\section{RHEUM}

Colliniamum, white lacinated leaves, very ornamental. . . . . .\$0.10

Emodii (macrophyllum) dark leaves.................. . . . . . . .

Giganteum, very robust...................... . 10

$\$ 0.20$

$\$ 0.40$

Officinale, very effective.................... 10

Palmatum tanguticum, brown-red foliage.

\section{RUDBECKIA (Cone Flower)}

Fulgida, orange-yellow, free flowering.............\$0.20

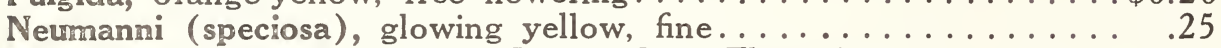

Purpurea (Echinacea), (Giant Purple Cone Flower) ........... . .50

Bicolor, golden yellow, dark brown centre............... . 10

\section{SALVIA}

Farinacea, pale blue flowers...................\$0.20 \$0.40

\section{SAPONARIA}

Ocymoides, lovely, pinkish-rose..................\$0.20 \$0.40

$\$ 0.80$

\section{SAXIFRAGA (Megasea)}

Cordifolia, rose, large flowers. . . . . . . . . . . . . . . . . \$2.00

Decipiens grandiffora, white, large flowered............. .40

\section{SCABIOSA (Pincushion)}

Caucasica, blue .....................................

Japonica, lilac, beautiful

\section{SEDUM}

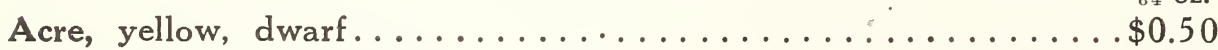

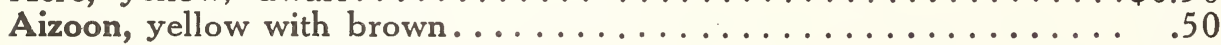

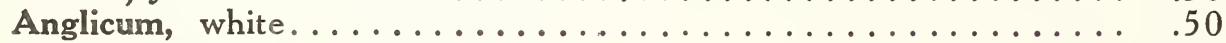

Ewersii, dark rose, large leaved.................. 1.00

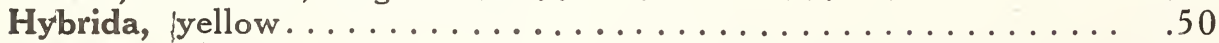

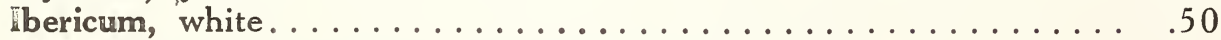

Middendorfii, yellow flowers and dark leaved . . . . . . . . . . . . . .

Purpurascens, with large, dark foliage, flowers purple........ . . 50

Reflexum, yellow.......................... . 50

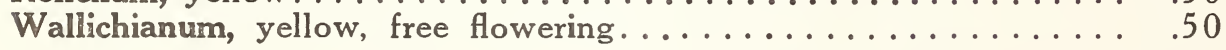

\section{SEMPERVIVUM}

Annuum, ............... Barboreum album, ...

Brachycaulum, ...... Canariensis, ....... Cholochrysum, Gracillis, ........ Haworthii, ....... Tabulacfornis 
Canadensis, golden yellow, beautiful............... $\$ 0.50$

Spectabilis, golden yellow, sweet scented... . . . . . . . . . . . .75

Virgo-Aurea Alpestris, early flowering, yellow

$1 / 2$ oz. $\quad 1 \mathrm{oz}$.

$\$ 1.00$

1.50

.50

3.00

1.00

\section{STACHYS}

Alpina (Alpine Hedge Nettle), dark rose, leave white....... . \$0.20

STATICE (Sea Lavender)

Latifolia, blue, fine for bouquets. . . . . . . . . . . . . \$0.75

Brassicaefolia, magnificent market plant.............. .75

Tatarica, white, best for dying

.25

$\$ 1.50$

$\$ 3.00$

1.50

3.00

1.00

\section{STENACTIS}

Speciosa, blue, beautiful for cutting...............\$0.25

STOKESIA (Stokes Aster)

Cyanea, flowers blue, like a giant cornflower, pretty........\$0.75

TRITOMA (Torch Lily, Flame Flower or Red Hot-Poker)

Uvaria grandiffora, flowers large coral-red, fading to orange . . . . . \$0.75 Tuckii (May Queen), dark rose................... . 50

\section{TROLLIUS}

Asiaticus, dark orange ................. \$1.00

Orangeball, large flowering, light yellow............. 1.25

Earliest of All, golden yellow................... 1.50

$1 / 4$ oz.

\section{VALERIANA (Garden Heliotrope)}

Alba, white, free flowering, for bouquets. . . . . . . . . . . . 0.20

Officinalis, rose-tinted white flowers, sweet scented.......... . 20

\section{VERBASCUM}

Harkness, new species, flowers sulphur yellow. . . . . . . . .\$0.20 \$0.40

\section{VERONICA (Speedwell)}

Amethystina, blue....................... \$0.80

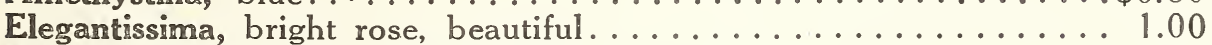

$\$ 1.60$

$\$ 3.00$

2.00

3.80

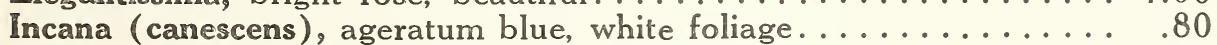

1.60

3.00

Longifolia, light blue, glaucous, blue, foliage............. . . . . . . . .

Hendersonii nana, dark blue................. 40

\section{VIOLA (Violet)}

Cornuta Hybrida, white perfection...............\$0.50 
Cornuta Hybrida Purple King ....................\$0.50

Blue Perfection.

Fairy Queen, light blue with white margin

Firmament, sky blue. .....................

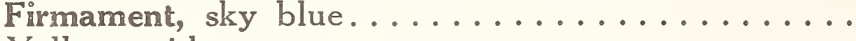

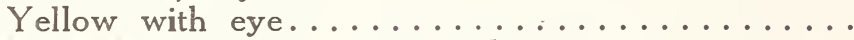

Lord Beaconsfield, purple violet. . . . . . . . . . .

Lutea splendens, pure yellow..............

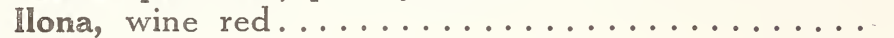

Rose Queen, rose salmon...............

Red and Rose varieties.................

Black Prince.

Sulphureum

Striata, striped variety.

Violet Blue. .

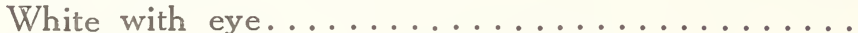

Adnirabilis, 3 and 5 blotched hybrids.

Rosea.

Best mixed.
$1 / 4$ oz.
$\$ 0.50$

.50

.50

.50

.50

.50

.50

.50

.50

.50

.50

.50

.50

.50

.50

.50

.50

.50
$1 / 2$ oz.

$\$ 1.00$

1.00

1.00

1.00

1.00

1.00

1.00

1.00

1.00

1.00

1.00

1.00

1.00

1.00

1.00

1.00

1.00

1.00
1 oz.

$\$ 2.00$

2.00

2.00

2.00

2.00

2.00

2.00

2.00

2.00

2.00

2.00

2.00

2.00

2.00

2.00

2.00

2.00

2.00

\section{WAHLENBERGIA (Platycodon)}

Grandiflora, Blue, very large flowering . . . . . . . . . . . \$0.75

White

\section{ORNAMENTAL GRASSES (Perennials)}

Andropogon argenteum (Silvery white beard Grass), with silvery foliage formosum, (Handsome Beard Grass).

$1 / 2$ oz.

$\$ 0.75$

2.75

Ceratochloa pendula. Fine for bouquets.

Elymus giganteus (Giant Bunch Grass)

Eulalia japonica (Japanese Eulalia), charming giant grass, very

handsome as single specimens.................... .50

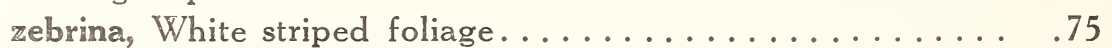

Festuca alpina superba (Superb Alpine Fescue Grass), pretty . . . . . . . . . .50

crinum ursi, fine border grass.

glauca (Glaucious Fescue Grass), for borders.

viridis (Green Fescue Grass), very fine, for borders

Gymnothrix Japonica (Bunch Grass).

latifolia, very decorative.

Gynerium argenteum (Silver Pampas Grass)

jubatum, pretty

Koeleria setacea, Fine border grass.

Isolepis gracillis (Slender Maiden Hair), for pots

Lasiagrostis argentea. Splendid for bouquets. .

Melica altissima (Tall Pearl Grass), silvery-white.

$$
\text { atropurpurea }
$$

Panicum virgatum (Twiggy Panick Grass), pretty for bouquets.

altissimum (Guinea Grass), tall growing ornamental grass,

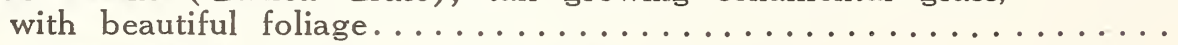

plicatum (Plaited-leaved Panick Grass), for pots...............

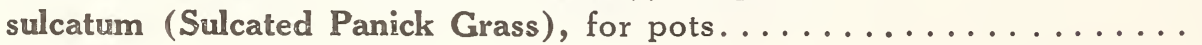

Stipa gigantea.

splendens

Uniola latifolia, pretty for bouquets.
.75
1 oz.

$\$ 1.50$

5.00

.50

.50

1.00

1.50

1.00

1.50

3.00

1.00

1.30

1.50

1.50

1.70

1.30

8.00

1.00

.80

.80

1.00

1.00

1.50

1.50

1.80

1.50

1.00 
Abies, Fir

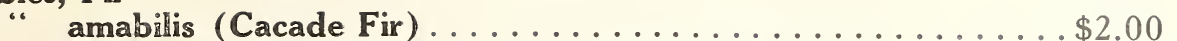

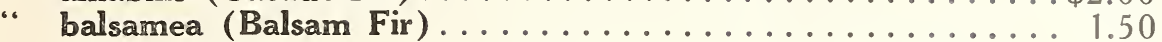

“ brachyphylla ......................... 1.50

“ cephalonica (Greek Fir) . . . . . . . . . . . . . . . 1.50

“ concolor (White Fir) . . . . . . . . . . . . . . 1.25

“ grandis (Great Silver Fir) . . . . . . . . . . . . . . . . 2.00

“ nobilis (Noble Fir) . . . . . . . . . . . . . . . . 2.50

“ nordmanniana (Nordmann Fir) . . . . . . . . . . . . 1.50

“ pectimata (European Silver Fir) ................ .75

$1 / 2 \mathrm{lb} . \quad 1 \mathrm{lb}$.

$\$ 4.00 \$ 8.00$

$3.00 \quad 6.00$

$3.00 \quad 6.00$

$3.00 \quad 6.00$

$2.50 \quad 5.00$

$4.00 \quad 8.00$

$5.00 \quad 10.00$

$3.00 \quad 6.00$

$1.50 \quad 3.00$

Cedrus, Cedar

“ atlantica (Atlas Cedar) ....................... 1.25

“ Deodara (Himalaya Cedar) . . . . . . . . ......... 1.50

$2.50 \quad 5.00$

$3.00 \quad 6.00$

Chamaecyparis, Cypress

“Lawsoniana (Lawson Cypress, domestic) . . . . . . . . . . . 1.25

2.50

5.00

“ obtusa (Hinoki Cypress) ...................... 2.00

4.00

8.00

pisifera (Sawara Retinospora)

2.00

4.00

Cupressus, Cypress (True Cypress)

s. fastigiata (Italia, imported) .............. 1.50

3.00

6.00

Juniperus, Juniper

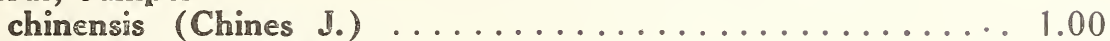

2.00

4.00

" communis (Common J.)

Sabina (Salbin)

1.50

3.00

2.50

5.00

Larix, Larch europaea (European Larch).

Picea, Spruce

“ Alcockiana (Alcock S.) . . . . . . . . . . . . . . . . . . 2.00

4.00

8.00

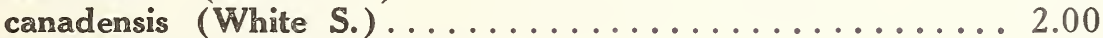

4.00

8.00

“ Engelmannii (Engelmann S.) .................... 2.00

4.00

8.00

exelsa (Norway S.)

.75

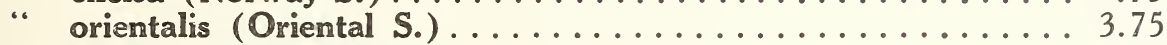

1.50

3.00

7.50

15.00

pungns (Blue Spruce) .................... 2.00

4.00

8.00

Pinus, Pine

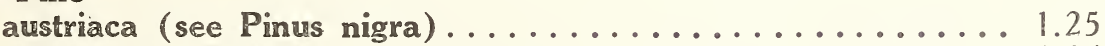

2.50

5.00

2.50

5.00

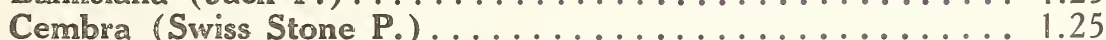

2.50

5.00

montana (Swiss Mountain P.) . . . . . . . . . . . . . 1.00

2.00

4.00

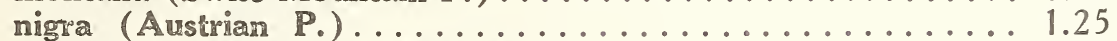

2.50

5.00

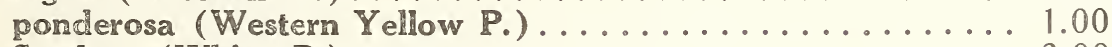

2.00

4.00

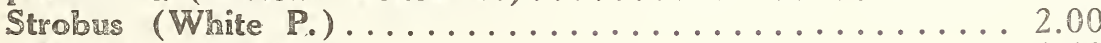

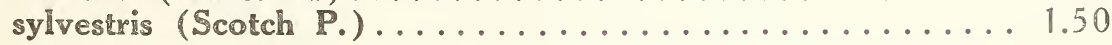

4.00

8.00

3.00

6.00

Thuja, Arborvitae

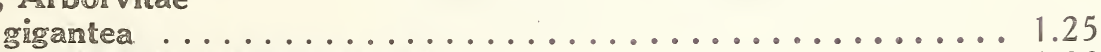

2.50

5.00

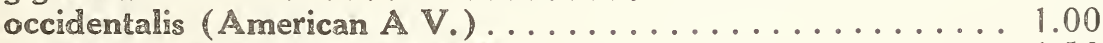

2.00

4.00

orientalis aurea (Golden O.AV)

1.50

3.00

6.00

$2.50 \quad 5.00$

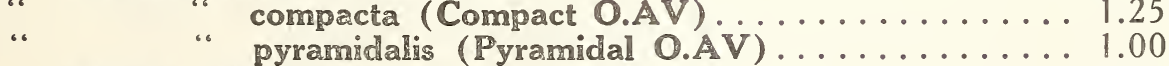

plicata (Giant AV).

2.50

2.00

4.00

5.00

10.00

Tsuga, Hemlock

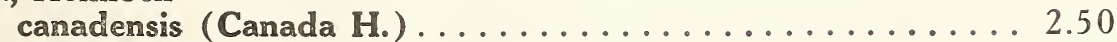

diversifolia (Japanese H.) . . . . . . . . . . . . . 3.00

5.00

10.00

sieboldi (Siebold H.)

3.00

6.00

12.00

6.00

12.00

Sciadopytis Vert.

1.50

3.00

6.00 


\section{VEGETABLE-SEEDS}

\section{BEANS-Dwarf (Buschbohnen)}

Green-podded Varieties:

Ilsenburg

$1 / 4 \mathrm{lb}$.

$1 / 2 \mathrm{lb}$.

$1 \mathrm{lb}$.

Emperor William......................... 25

$\$ 0.35 \$ 0.60$

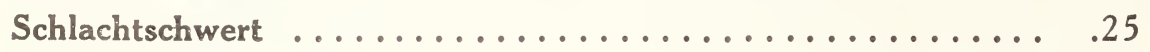

$.40 \quad .75$

Sugar, Hinrich Giant..................... . 20

$.40 \quad .75$

$.35 \quad .60$

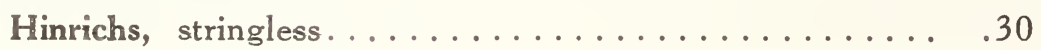

$.55 \quad 1.00$

Wax podded Varieties:

Rhineland, Novelty 1926, very early.............. .40

$.70 \quad 1.20$

Flageolet ............................ . 25

$.45 \quad .80$

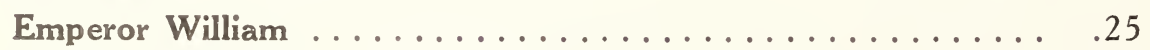

$.45 \quad .80$

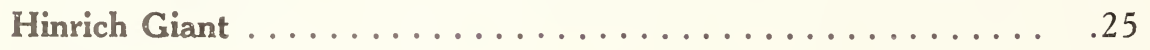

$.40 \quad .75$

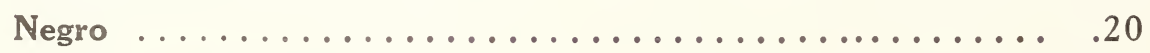

$.35 \quad .70$

Broad Beans: (Puffbohnen)

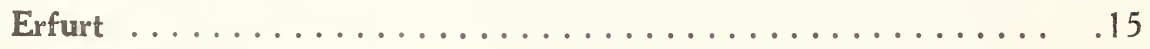

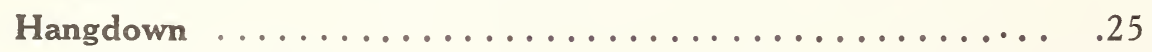

\section{BEETS (Roteruben)}

Erfurt, Long Dark Blood Red.....................\$0.20

Egyptian, Turnip, Rooted, Dark Red.................. . 30

\section{BRUSSELS SPROUTS (Rosenkohl)}

Gonsenheim

\section{WHITE CABBAGE (Weisskraut)}

Copenhagen Market...................... \$0.25

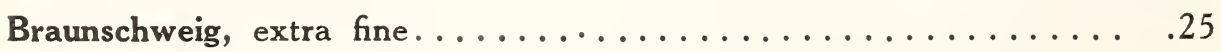

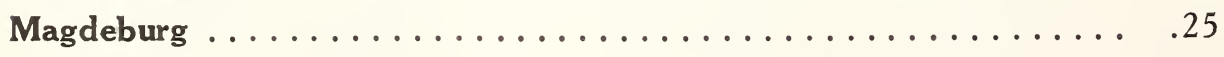

$1.40 \quad 2.50$

\section{RED CABBAGE (Rotkraut)}

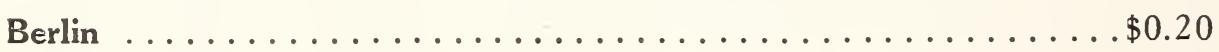


Advent

Ulm, dwarf
$1 \mathrm{oz}$

$\$ 0.30$

.25
$1 / 2 \mathrm{lb}$.

$\$ 1.60$

1.50
$1 \mathrm{lb}$.

$\$ 3.00$

2.80

CAULIFLOWER (Blumenkohl)

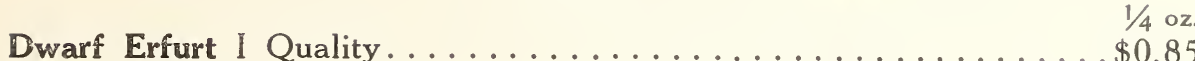

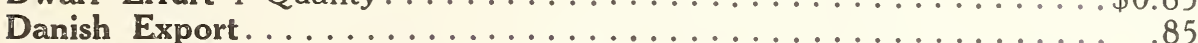

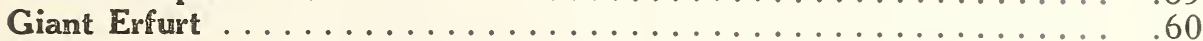

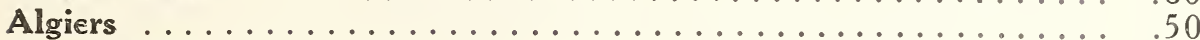

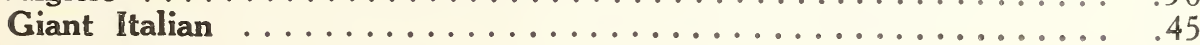

$1 / 2$ oz. $\quad 1 \mathrm{oz}$.

$\$ 1.60 \$ 3.00$

$1.60 \quad 3.00$

$1.10 \quad 2.00$

$.90 \quad 1.75$

$.80 \quad 1.50$

\section{CARROT (Carrotten)}

Frankfort, Half Long.................... 1 oz

Gonsenheim, Half Long. ....................... .25

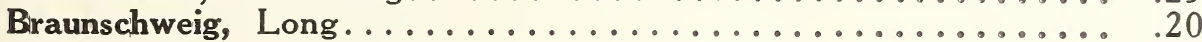

Erfurt, Long.............................. 20

$1 / 2 \mathrm{lb} . \quad \mathrm{llb}$.

$\$ 0.95 \$ \$ 1.75$

$1.30 \quad 2.50$

$.90 \quad 1.60$

$.90 \quad 1.60$

\section{CELERIAC (Knollen-Sellerie)}

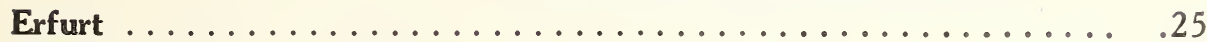

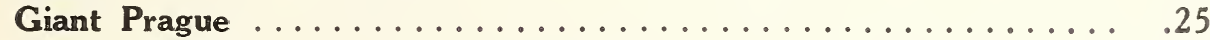

Apple-shaped

$1.30 \quad 2.50$

$1.30 \quad 2.50$

$1.30 \quad 2.50$

\section{CELERY (Beich Sellerie)}

Easy Blanching. ........................... . $\$ 0.45$

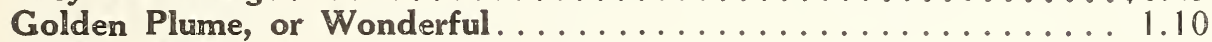

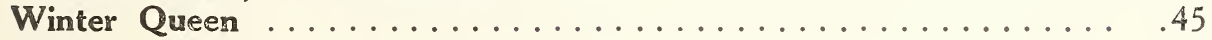

\section{CUCUMBER (Gurken)}

Erfurt, Half Long.......................... . . . . . . . 30

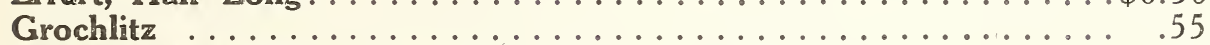

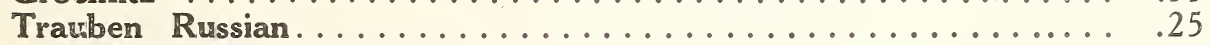

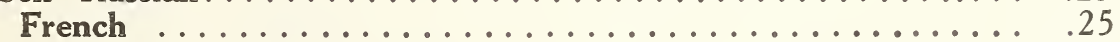

EGG PLANT

Black Beauty ......................... $\$ 0.50$

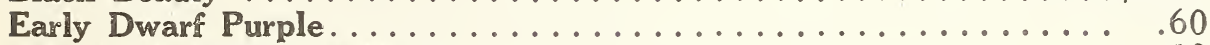

New York Improved Purple..................... . 50

$\$ 3.00 \$ 6.00$

$3.50 \quad 7.00$

$3.00 \quad 6.00$

\section{ENDIVE (Endivien)}

Yellow Escariol, Winter...................... \$0.25

Green Curled v. Meaux, Winter.................... 20

\section{KALE OR BORECOLE (Blatterkohl)}

Semi-Dwarf Green Moss-Curled.

\section{KOHL RABI (Kohl-Rabi)}

Erfurt Dreienbrunnen, White....................\$0.25

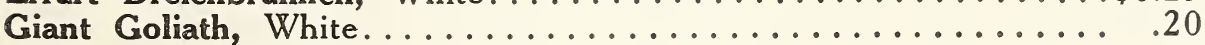


LETTUCE (Kopfsalat)

Dresden, Large Yellow....................... \$0.20

May King.

ONION (Zwiebel)

Giant Zittau, Yellow.

PARSNIP (Pastinake)

Long, White............................ \$0.10

Round, White.

$\$ 0.50$

$\$ 0.90$

$.55 \quad 1.00$

PARSLEY (Petersilie)

Moss, Curled

$\$ 0.15$

Rooted, Long .

$\$ 0.55$

$\$ 1.00$

.90

PEAS-Round-seeded (Pahlerbsen)

De Grace.

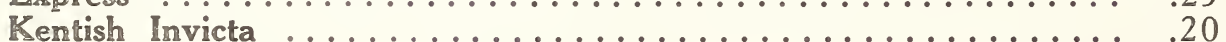

PEAS-Wrinkled-seeded (Markerbsen)

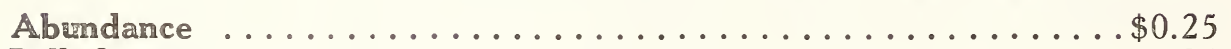

Belladonna

Telephone

Senator

\section{PEPPER}

Chinese Giant

Early Dwarf Red Squash

Napolitan

Red Chili .............................. 50

Sweet Mountain, or Mammoth
1 oz.

$\$ 0.65$

.50

.50

.50
$1 / 2 \mathrm{lb}$.

$\$ 3.00$

2.50

2.50

2.50

2.50
$1 \mathrm{lb}$. $\$ 6.00$ 5.00 5.00 5.00 5.00

\section{RADISH (Radies)}

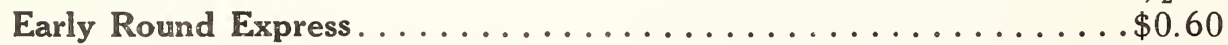

\section{RADISH (Rettich)}

Summer, Salvator, White 
Giant Russian

\section{SPINACH (Spinat)}

Giant Eskimo

\section{SWISS CHARD (Mangold od. Beisskohl)}

Swiss extra

Silver
$1 \mathrm{oz}$. $\$ 0.15$
$1 / 2 \mathrm{lb}$

$\$ 0.55$
$1 \mathrm{lb}$. $\$ 1.00$ 1.00

\section{TURNIP (Kohl-od. Steckruben)}

Yellow, Hoffmanns Giant

$1 / 2 \mathrm{lb}$

$\$ 0.40$

Giant, Red Gray, top

Schmalz

Chalk's Jewel

Dwarf Stone

Earliana

Greater Baltimore

John Baer

Ponderosa

Stone

Yellow Cherry
1 oz. $1 / 4 \mathrm{lb}$.

$\$ 0.50$

\section{SWEET, POT AND MEDICINAL HERBS}

Anise, Thuringia

$1 / 4$ oz.

Artemisia vulgaris (Beifuss)

Basil, fine green curled (Basilikum)

Caraway (Kummel) . .

Cochlearia officinalis (Loffelkraut)

$\$ 0.20$

Cress, Garden (Gartenkresse)

Water (Erfurter Brunnenkresse)

Chervil (Kerbel)

Dill (Dill)

Fennel (Fenchel)

Garden, Burnet (Pimpinelle)

Garden, Melde

Lavender (Lavendel)

Levisticum, officinale ( Liebstock)

Majoram, French (Franzosischer Majoram)

Portulaca, Yellow (Portulak)

Rosemary (Rosmarin)

Savory, Annual (Bohnen-od. Pfefferkraut)

Sage (Salbei)

Sorrel (Sauerampfer)

Tarragon, Russian (Esdragon)

Thyme, Winter (Thymian)

Wormwood (Wermut)
$1 / 2$ oz. $\quad 1$ oz.

$\$ 0.10 \$ 0.20$

$.40 \quad .80$

$.15 \quad .25$

$.15 \quad .25$

$.40 \quad .75$

$.15 \quad .30$

$.45 \quad .90$

.15

$.10 \quad .20$

$.10 \quad .20$

$.15 \quad .25$

$.15 \quad .25$

$.15 \quad .25$

$.40 \quad .75$

$.15 \quad .30$

$.15 \quad .25$ 


\section{Florist's and Nursery Knives Made by}

\section{S. Kunde E Sohn, Dresden, Germany}

22H [. S.KUNDE SOSOH DRESOEN,

No. 1 Full Length $6 \frac{1}{2}$ ir ch $^{\$ 2.75}$

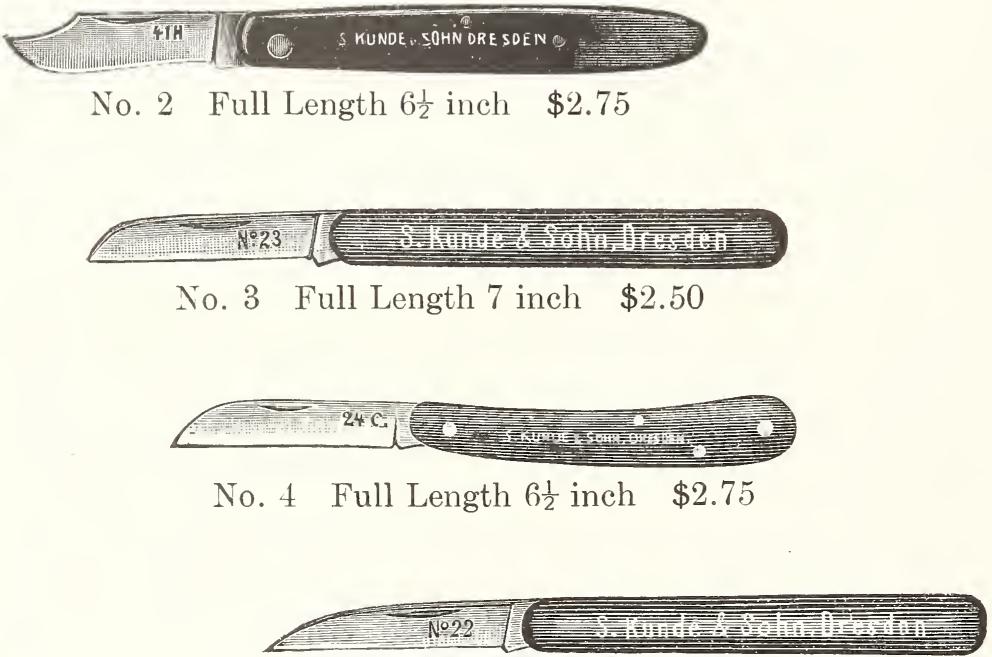

No. 5 Full Length $6 \frac{1}{2}$ inch $\$ 2.50$

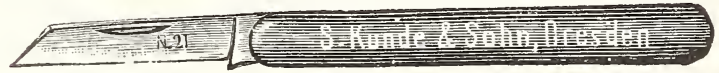

No. 6 Full Length $6 \frac{1}{2}$ inch $\$ 2.50$

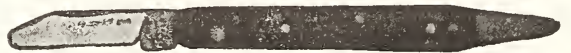

No. 7 Full Lentgh 6 inch $\$ 1.50$

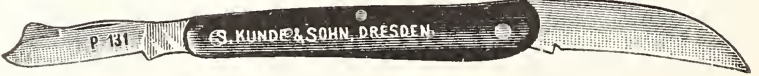

No. 8 Full Length 9 inch $\$ 3.00$

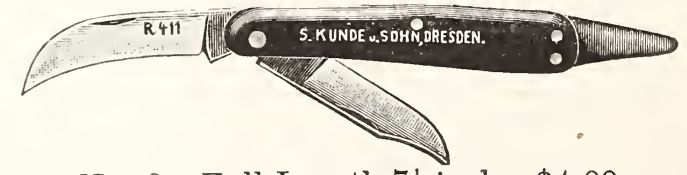

No. 9 Full Length $7 \frac{1}{2}$ inch $\$ 4.00$ 


\section{Florist's and Nursery Knives}

Made by

\section{S. Kunde \& Sohn, Dresden, Germany}

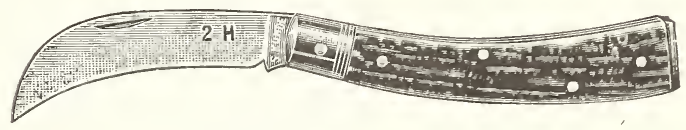

No. 10 Full Lenght 7 inch $\$ 4.50$

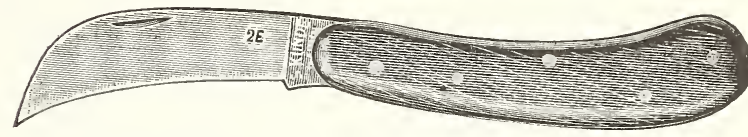

No. 11 Full Length 8 inch $\$ 3.75$

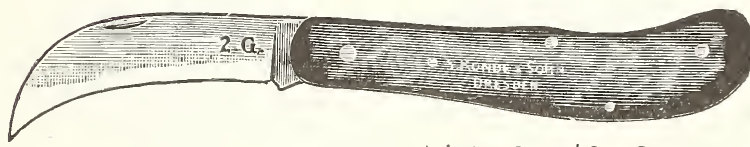

No. 12 Full Lenght $7 \frac{1}{2}$ inch $\$ 3.50$

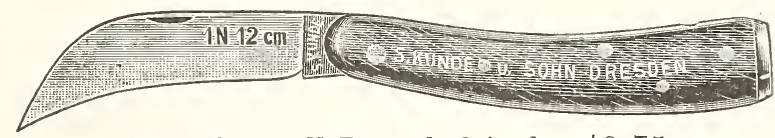

No. 13 Full Length 8 inch $\$ 3.75$

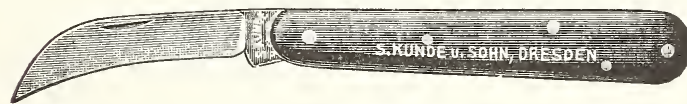

No 14 Full Length $6 \frac{1}{2}$ inch $\$ 2.75$

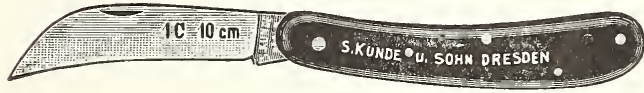

No. 15 Full Length 7 inch $\$ 2.75$

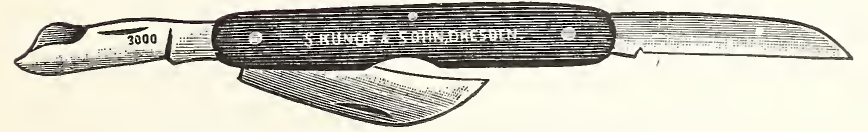

No. 16 Full Length $8 \frac{1}{2}$ inch $\$ 4.50$

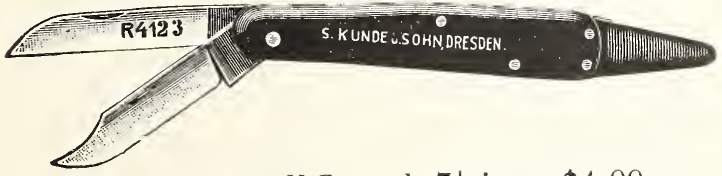

No. 17 Full Length $7 \frac{1}{2}$ inc $\$ 4.00$

Prices include free delivery to you. Remittance must accompany order. No C. O. D. The knives are sold on account of high import duty, at practically no profit at all. $5 \%$ discount on orders for 3. knives or more. 


\begin{tabular}{|c|c|c|}
\hline Page & Page & $\mathrm{Pa}$ \\
\hline .49 & Cineraria & Julienne \\
\hline & & us \\
\hline & ea & Bo \\
\hline 3 & hlearia & \\
\hline 3 & Co & abi \\
\hline clinium & elina & \\
\hline . 39 & C & \\
\hline 4 & & us \\
\hline mma $\ldots$ & & er \\
\hline . .3\&39 & er & \\
\hline us & $\ldots$ & \\
\hline & is $\ldots$ & em \\
\hline e & C & $\mathrm{dm}$ \\
\hline & um $\ldots 1$ & L \\
\hline $\mathrm{m}$ &. $.18 \&$ & \\
\hline gia & is. & \\
\hline & & us $\ldots \ldots 2$ \\
\hline & oteca & $s \ldots$ \\
\hline & & m .. \\
\hline cir & um & d. . \\
\hline 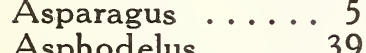 & $\mathrm{D}$ & aria .. \\
\hline$\dot{6}$ & 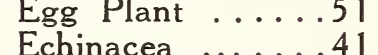 & o \\
\hline
\end{tabular}

Aster ...5, 6, $7 \& 40$

Astilbe .......440

Aubrietia .....440

Balsam ........12

Basil ........53

Beans ........50

Beets .......50

Begonia $8,9,10,11,12$

Bellis .......12

Bidens .......40

Bocconia .......440

Brussels Sprouts. 50

Cabbage ...50\&51

Calceolaria ..12\&13

Calendula .....13

Calliopsis .....13

Campanula ....440

Candytuft . . 13\&40

Cannabis .....13

Caraway .......53

Carnations .....14

Carrots .......51

Cauliflower .....51

Cedrus .......49 49

Celosia .......14

Celeriac ......51

Celery ........51

Centaurea ...14\&40

Cerasticum .....440

Chamaepeuge ....40

Chamaecyparis ...49

Chelone ......41

Cheiranthus ....40

Chervil ........53

Chrysanthemum ..15
Echinacea .......4

Echinops .....42

Endive .......51

Erigeron ......41

Eryngium .....44

Erysimum .....44

Escholtzia .....18

Fennel ........53

Funkia .......42

Ferns ........37

Gaillardia . . 19\&42

Gerbera .......42

Gesneria .......19

Geum ........442

Gladiolus .......42

Gloxinia .......19

Gnaphalium ....442

Godetia .......19

Gypsophila . . 19\&42

Helenium ......43

Helianthemum ...43

Helianthus ...20\&43

Helichrysum ....20

Heliopsis .....43

Helleborus .....43

Heliotropium ....20

Hemerocallis ...44

Hesperis ......43

Heuchera ......43

Hibiscus ......43

Hollyhook .....43

Hyacinthus .....43

Hypericum ....44

Iberis .......44

Iris ........44
Mesembryanthemum

Mignonette ......21

Mimulus ......21

Mirabilis ......21

Morning Glory .22

Myosotis ...22\&44

Nicotiana ......22

Nigella .......22

Oenothera .....22

Onion ........52

Ornamental Grasses $38 \& 48$

Paeonia ......44

Pansies. 23, 24, \&25

Papaver ...22\&45

Parsley ......52

Parsnip .......52

Peas ........55

Pentstemon ....45

Perilla .......25

Pepper .......52

Petunia

$$
26,27,28 \& 29
$$

Phlox .....25\&45

Fhysális .....45

Fhytolacca ....445

Physostegia ....45

Picea .......449

Pinus ........49

Pimpinelle .....53

Platycodon ......45

Portulaca ... 31\&53

Primula ....30\&45

Potentilla .....45
Page

Pueraria ......31

Pyrethrum ...31\&45

Radish .......52

Ranunculus .....46

Rheum ......46

Rhodanthe ....31

Ricinus ........31

Rosemary .....53

Rudbeckia .....46

Sage .........53

Salpiglossis .....31

Salvia .... . 31\&46

Saponaria ...31\&46

Saxifraga .....46

Savory .......53

Scabiosa ...31\&46

Schizanthus .....32

Sciadopytis ....49

Sedum .......46

Sempervivum ....46

Silene ........32

Scorzonera .....53

Smilax ........32

Spinach .....53

Solidago ......47

Sorrel ........53

Stachys ......47

Statice ....32\&47

Stenactis ......47

Stocks .....32\&33

Stokesia ......47

Swainsonia .....33

Sweet Peas .....34

Swiss Chard.....53

Tagetes .......33

Tarragon .....53

Thuja .......49

Thunbergia ....33

Thyme .......53

Tomato ......53

Torenia .......35

Tritoma .....44

Trollius ......47

Tropaeolum ....35

Tsuga .......49

Turnip .......53

Valeriana .....47

Verbascum .....47

Verbena .......35

Veronica .....47

Vinca ........36

Viola .....47\&48

Wahlenbergia ..48

Wallflower .....36

Vormwood ....53

Xeranthemum ...36

Zinnia ......36 



\section{.}

